\title{
A Finite Presentation of Universal Coverings of Lie Tori
}

\author{
by \\ Saeid Azam, Hiroyuki Yamane and Malihe YousofzadeH
}

\begin{abstract}
Using the well-known recognition and structural theorem(s) for root-graded Lie algebras and their universal coverings, we give a finite presentation for the universal covering algebra of a centerless Lie torus of type $X \neq A, C, B C$. We follow a unified approach for the types under consideration.
\end{abstract}

2010 Mathematics Subject Classification: 17B67, 17B65, 17B35, 17B70.

Keywords: extended affine Lie algebras, Lie tori, root graded Lie algebras, finite presentation, universal enveloping algebra.

\section{$\S 1$. Introduction}

The notion of a Lie torus arises in the study of extended affine Lie algebras which are natural generalizations of finite-dimensional simple Lie algebras and affine Lie algebras. Extended affine Lie algebras and Lie tori have been under intensive investigation in recent years.

It is well understood that the study of an extended affine Lie algebra, in any aspect, somehow relates to the study of its core modulo the center, called the centerless core. Here is the place where the notion of Lie tori arises; the centerless core of an extended affine Lie algebra is a centerless Lie torus and conversely any centerless Lie torus is the centerless core of an extended affine Lie algebra ([Yos] $)$.

Communicated by M. Kashiwara, Received July 15, 2009. Revised December 1, 2009.

S. Azam: School of Mathematics, Institute for Research in Fundamental Sciences (IPM), P.O. Box 19395-5746, Tehran, Iran, and Department of Mathematics, University of Isfahan, P.O. Box 81745-163, Isfahan, Iran;

e-mail: azam@sci.ui.ac.ir

H. Yamane: Department of Pure and Applied Mathematics, Graduate School of Information Science and Technology, Osaka University, Toyonaka, Osaka, 560-0043, Japan;

e-mail: yamane@ist.osaka-u.ac.jp

M. Yousofzadeh: Department of Mathematics, University of Isfahan, P.O. Box 81745-163, Isfahan, Iran;

e-mail: ma.yousofzadeh@sci.ui.ac.ir

(C) 2010 Research Institute for Mathematical Sciences, Kyoto University. All rights reserved. 
Lie tori as well as extended affine Lie algebras are defined axiomatically ( AABGP), and their structures are well studied. In particular, it is known that a Lie torus is a root graded Lie algebra, a notion which turns out to be essential in the structure theory of extended affine Lie algebras and Lie tori.

The structure of root graded Lie algebras of reduced types is determined up to central isogeny in $[\mathrm{BM}$ ] and $[\mathrm{BZ}$. Namely a root graded Lie algebra is centrally isogenous to a Lie algebra having a prescribed structure coming from some known algebra constructions, including the so called generalized Tits construction (see Section 2). We refer to these results as Recognition Theorem(s) (see Theorem 2.1). The Recognition Theorem together with several theorems from [ABG], $[\mathrm{BZ},[\mathrm{AG}]$ and $\mathrm{BGK}$ enables us to decompose the universal covering algebra $\mathfrak{A}$ of the centerless core of an extended affine Lie algebra of type $X=B_{\ell}(\ell \geq 3)$, $D_{\ell}(\ell \geq 4), E_{6}, E_{7}, E_{8}, F_{4}$ and $G_{2}$ as

$$
\mathfrak{A}=(\mathcal{G} \otimes \mathcal{A}) \oplus\left(\mathcal{V} \otimes \mathcal{A}^{m}\right) \oplus \mathcal{D}
$$

where $\mathcal{G}$ is a finite-dimensional simple Lie algebra of type $X, \mathcal{V}$ is an irreducible $\mathcal{G}$-module whose highest weight is the highest short root of $\mathcal{G}, \mathcal{A}$ is the algebra of Laurent polynomials in several variables, $\mathcal{A}^{m}$ is the direct sum of $m$ copies of $\mathcal{A}$, and $\mathcal{D}$ is a known subalgebra of $\mathfrak{A}$ related to the inner derivations of the so called coordinate algebra of $\mathfrak{A}$. We do not consider type $B_{2}$ in this work as the structure theory of a root graded Lie algebra of type $B_{2}=C_{2}$ in $[\mathrm{BZ}$ ] and $\mathrm{AG}$ ] is studied within $C_{\ell}(\ell \geq 2$ )-graded algebras. A similar presentation for type $C$ (and also type $A$ ) will be considered in a separate work.

In this work, we use decomposition $(*)$ to give a nullity-free finite presentation for the universal covering of the centerless core of a Lie torus of type $X \neq$ $A_{\ell}, C_{\ell}, B C_{\ell}$. Since the ingredients appearing in (*) and their algebra structures are completely known (see Theorems 2.3 and 2.2 , we are able to select a (finite) set of generators for $\mathfrak{A}$ and deduce a certain (finite) set of relations among them. This then motivates the generators and defining relations of our presented Lie algebra.

There have been several attempts to present a Lie torus (up to center) by a (finite) set of generators and relations. Historically, we may name the works of $\mathrm{Ka}$ ] and [MRY] for toroidal Lie algebras; $[\mathrm{SY}]$ and $[\mathrm{Yam}$ for elliptic Lie algebras (2extended affine Lie algebras); and YYou for Lie tori of type $B_{\ell},(\ell \geq 3)$. It is worth mentioning that the universal covering of the centerless core of a finite-dimensional simple Lie algebra or an affine Kac-Moody Lie algebra is just itself or its derived algebra, respectively. Therefore, for the finite or affine case the well-known Serretype presentation is in fact given for the universal covering of the centerless core. This might justify why we are considering the universal covering instead of a Lie torus itself. 
The paper is arranged as follows. In Section 2, we record some necessary background and results needed for describing the universal covering of the centerless core of a Lie torus in the form $(*)$. In brief, we establish some minor results regarding finite root systems and irreducible modules of finite-dimensional simple Lie algebras (Lemmas 2.1 2.2. The generalized Tits construction and basic properties of root graded Lie algebras are reviewed. The Recognition Theorem(s) from [BM] and [BZ] regarding the structure of root graded Lie algebras are recalled, and some results from $\mathrm{BGK}, \mathrm{AG}$ and $\mathrm{ABG}$ regarding the universal covering algebras of root graded Lie algebras and Lie tori are restated. Altogether, these enable us to deduce the decomposition $(*)$. We end the section by obtaining from $(*)$ a finite set of generators and a certain set of relations for $\mathfrak{A}$.

In Section 3, we introduce the generators and defining relations of our presented Lie algebra $\tilde{\mathcal{L}}$, associated to a Lie torus of type $X \neq A_{\ell}, C_{\ell}, B C_{\ell}$, and deduce certain immediate consequences, which will be essential for the proof of our main theorem (Theorem 4.1). For the convenience of the reader, we have shifted the proofs of some of these results containing complicated technicalities to Section 5 . Section 4 is devoted to the proof of our Main Theorem (Theorem 4.1): the universal covering algebra of the centerless Lie torus under consideration is isomorphic to its corresponding presented Lie algebra $\tilde{\mathcal{L}}$. The proof is in several steps. In step 1 , we prove that $\tilde{\mathcal{L}}$ is root graded and we obtain some information regarding its center. In the remaining steps we complete the proof for simply laced types, $F_{4}$ and $G_{2}$, respectively. For type $B_{\ell}(\ell \geq 3)$, we refer the reader to You, though the proof for this type can also be deduced from our setting. This work continues the line of research started in $\mathrm{You}$ for type $B_{\ell}(\ell \geq 3)$; here we have enlarged our setting in order to have a unified approach for the types under consideration. Needless to say, in both works the defining generators and relations of the relevant presented Lie algebras are inspired from the algebra structures of the ingredients involved in the decomposition $(*)$.

For non-simply laced types, our presentation depends on certain crucial relations (see 2.13 - 2.15) among some structural data which are obtained using several Mathematica programs. These data arise from the Lie algebra structure on ingredients in $(*)$ (see 2.16) $)$. For the convenience of the reader we have posted a longer version of this paper at arXiv:math.QA/0906.0158; it contains an appendix furnishing the detailed information about the data mentioned above.

\section{§2. Terminology and prerequisites}

Throughout this paper we suppose that $R$ is an irreducible reduced finite root system of type $G_{2}$ or of rank greater than or equal 3 which is not of type $A, C$. 
By $R^{\times}$, we mean $R \backslash\{0\}$. All vector spaces and tensor products are taken over the field of complex numbers $\mathbb{C}$. For an algebra $A$ which is not a Lie algebra, by $[a, b]$, $a, b \in A$, we mean $a b-b a$. If $\alpha, \beta$ are two elements of a unital algebra $\mathcal{A}$ which is associative, alternative or Jordan, we denote by $D_{\alpha, \beta}$ the inner derivation of $\mathcal{A}$ based on $\alpha, \beta$; we also use $D_{\mathcal{A}, \mathcal{A}}$ to denote the $\operatorname{subspace}_{\operatorname{span}_{\mathbb{C}}}\left\{D_{\alpha, \beta} \mid \alpha, \beta \in \mathcal{A}\right\}$ of derivations of $\mathcal{A}[\mathrm{Sc}]$.

For a positive integer $\nu$, we denote by $A_{[\nu]}$ the commutative associative algebra of Laurent polynomials in $\nu$ variables $t_{1}, \ldots, t_{\nu}$ and we use $A_{[\nu]}^{m}$, for a positive integer $m$, to denote the direct sum of $m$ copies of $A_{[\nu]}$. Also by $A_{[\nu]}^{0}$ we mean the trivial vector space. For $\sigma=\left(n_{1}, \ldots, n_{\nu}\right) \in \mathbb{Z}^{\nu}$ we denote $t_{1}^{n_{1}} \ldots t_{\nu}^{n_{\nu}}$ by $t^{\sigma}$. We also make a convention that for elements $x, x_{1}, \ldots, x_{m}, y$ of a Lie algebra, by $\left[x_{1}, \ldots, x_{m}, y\right]$ we always mean $\left[x_{1}, \cdots\left[x_{m-1},\left[x_{m}, y\right]\right] \cdots\right]$. In this case if $m=0$ we interpret $\left[x_{1}, \ldots, x_{m}, y\right]$ as $y$; also by $\left[x^{m}, y\right]$ we mean $[x, \ldots, x, y]$ with $x$ appearing $m$ times.

In a vector space, we define $\sum_{i=1}^{m} \cdots$ to be zero if $m=0$. For simplicity of notation we denote the set $\{1, \ldots, k\}$ be $J_{k}, k$ a positive integer. For a Lie algebra $\mathcal{L}$, by $Z(\mathcal{L})$ we mean the center of $\mathcal{L}$, and by a centerless Lie algebra we mean a Lie algebra with trivial center. We start the paper by recalling some (in general) non-associative algebra constructions and some related theorems which are of use throughout this work.

\section{§2.1. General facts}

Suppose that $\mathcal{G}$ is a finite-dimensional simple Lie algebra over $\mathbb{C}$ of type $X \neq A, C$ and of rank $\ell$, and $R$ is the corresponding irreducible finite root system with a root base $\Delta$. Let $R^{+}, R_{\mathrm{sh}}^{+}\left[R_{\mathrm{sh}}\right]$ and $R_{\mathrm{lg}}^{+}\left[R_{\mathrm{lg}}\right]$ be the set of positive roots, the set of positive short roots [the set of short roots], and the set of positive long roots [the set of long roots], respectively. If $X$ is simply laced we assume by convention that $R_{\mathrm{lg}}^{+}=\emptyset$. Also assume that $n$ is the number of positive roots. We arrange the set of positive roots as follows:

$$
\overbrace{\alpha_{1}, \ldots, \alpha_{n_{\ell}}}^{R_{\text {lg }}^{+} \cap \Delta}, \overbrace{\alpha_{n_{\ell}+1}, \ldots, \alpha_{\ell}, \ldots, \alpha_{n_{s}}}^{R_{\text {sh }}^{+}}, \overbrace{\alpha_{n_{s}+1}, \ldots, \alpha_{n}}^{R_{\text {lg }}^{+} \backslash \Delta} .
$$

Clearly if there is no long root $\left(n_{\ell}=0\right)$, then $n=n_{s}$. We may assume that $\alpha_{n_{s}}$ is the highest short root and $\alpha_{n}$ is the highest root. For $t \in J_{n}$, we fix $e_{t} \in \mathcal{G}_{\alpha_{t}}$ and $f_{t} \in \mathcal{G}_{-\alpha_{t}}$ such that $\left(e_{t}, h_{t}:=\left[e_{t}, f_{t}\right], f_{t}\right)$ is an $\mathfrak{s l}_{2}$-triple.

Using the finite-dimensional theory, one can prove the following lemma:

Lemma 2.1. (i) If $R_{\mathrm{lg}} \neq \emptyset$, then $R_{\mathrm{sh}} \subseteq R_{\mathrm{lg}}+R_{\mathrm{sh}}$.

(ii) For $\alpha \in R_{\mathrm{sh}}^{+}$, there is $\beta \in R_{\mathrm{sh}}^{+}$such that $\alpha+\beta \in R$. 
Lemma 2.2. Let $\mathcal{W}$ be an irreducible finite-dimensional $\mathcal{G}$-module.

(i) Suppose that the highest weight of $\mathcal{W}$ is $\lambda \in R$ and take $\Pi$ to be the set of weights of $\mathcal{W}$. Then for $\mu \in \Pi \backslash\{0\}$, there are $\alpha_{i_{1}}, \ldots, \alpha_{i_{p}} \in R^{+}$such that

$$
\mathcal{W}_{\mu}=\mathcal{G}_{-\alpha_{i_{p}}} \cdots \mathcal{G}_{-\alpha_{i_{1}}} \cdot \mathcal{W}_{\lambda} \quad \text { with } \quad \alpha_{i_{k}} \neq \pm\left(\lambda-\sum_{r=1}^{k-1} \alpha_{i_{r}}\right) \text {. }
$$

(ii) Suppose that the set of weights of $\mathcal{W}$ coincides with the set of short weights and consider the weight space decomposition $\mathcal{W}=\mathcal{W}_{0} \oplus \sum_{i=n_{\ell}+1}^{n_{s}} \mathbb{C} w_{ \pm i}$ in which for $n_{\ell}+1 \leq i \leq n_{s}, w_{ \pm i}$ is a weight vector of weight $\pm \alpha_{i}$. Then for $n_{\ell}+1 \leq i \leq n_{s}$

$$
e_{i} \cdot f_{i} \cdot w_{i}=2 w_{i}, \quad e_{i} \cdot f_{i} \cdot f_{i} \cdot w_{i}=2 f_{i} \cdot w_{i}, \quad \mathbb{C} e_{i} \cdot w_{-i}=\mathbb{C} f_{i} \cdot w_{i}
$$

and $\mathcal{W}_{0}$ is spanned by $f_{i} \cdot w_{i}, n_{\ell}+1 \leq i \leq \ell$.

Proof. (i) We use a simple argument from the finite-dimensional theory.

(ii) We first note that if $\beta \in \Pi \backslash\{0\}$, then $\operatorname{dim}\left(\mathcal{W}_{\beta}\right)=1$, and if $\beta \in \Pi$ and $\alpha \in R$ are such that $\alpha+\beta \in \Pi$, then $\mathcal{G}_{\alpha} \cdot \mathcal{W}_{\beta}=\mathcal{W}_{\alpha+\beta}$. Now let $\mathcal{U}\left(N^{-}\right)$be the universal enveloping algebra of $N^{-}:=\sum_{\alpha<0} \mathcal{G}_{\alpha}$. Then if $w$ is a highest vector, we have $\mathcal{U}\left(N^{-}\right) \cdot w=\mathcal{W}$. Also for each $\beta \in R^{+}$we have

$$
\mathcal{G}_{-\beta}=\left[\mathcal{G}_{-\beta_{s}}, \ldots, \mathcal{G}_{-\beta_{1}}\right] \quad \text { for some } \beta_{i} \in \Delta .
$$

Combining these facts and using the module action yields the required expression for $\mathcal{W}_{\mu}$ with $\mu \in R^{+}$. Note that the claim concerning $\alpha_{i_{k}}$ 's is automatically satisfied.

Next let $\mu \in-R^{+}$. Then there is $\lambda \in R^{+}$such that either $\mu-\lambda \in R^{+}$or $\mu+\lambda \in R^{+}$. Moreover if $\mu \in R_{\mathrm{sh}}^{+}$, then $\lambda$ can be chosen as an element of $R_{\mathrm{sh}}^{+}$such that $\mu+\lambda \in R$. We have

$$
\mathcal{W}_{\mu}= \begin{cases}\mathcal{G}_{-(\lambda+\mu)} \cdot \mathcal{W}_{-\mu} & \text { if } \lambda+\mu \in R^{+} \\ \mathcal{G}_{\lambda-\mu} \cdot \mathcal{G}_{\mu} \cdot \mathcal{W}_{\mu-\lambda} & \text { if } \lambda-\mu \in R^{+}\end{cases}
$$

Now we replace $\mathcal{W}_{\mu}$ or $\mathcal{W}_{\mu-\lambda}$ by an expression obtained from the previous step to get the required expression for $\mathcal{W}_{\mu}$. Note that from the way we found this expression for $\mathcal{W}_{\mu}$, it becomes clear that the claim concerning $\alpha_{j_{k}}$ 's holds.

2.1.1. Generalized Tits construction. Let $A$ be a unital commutative associative algebra and assume that $X$ is a unital algebra over $A$. A normalized trace on $X$ is an $A$-linear map $T: X \rightarrow A$ satisfying, for $x, x^{\prime}, x^{\prime \prime} \in X$,

$$
T(1)=1, \quad T\left(x x^{\prime}\right)=T\left(x^{\prime} x\right), \quad T\left(\left(x x^{\prime}\right) x^{\prime \prime}\right)=T\left(x\left(x^{\prime} x^{\prime \prime}\right)\right) .
$$


If $T$ is a normalized trace, the maps $t$ and $*$ defined by

$$
\begin{gathered}
t: X \times X \rightarrow A ;(x, y) \mapsto T(x y), \\
*: X \times X \rightarrow X ;(x, y) \mapsto x y-t(x, y) 1,
\end{gathered}
$$

are called, respectively, the trace form and the *-operator with respect to $T$. We use the same symbols $T, t$ and $*$ to denote the normalized trace, the trace form and the corresponding $*$-operator for different algebras. We also have $X=A 1 \oplus X_{0}$, where $X_{0}:=\{x \in X \mid T(x)=0\}$. Let $\operatorname{Der}_{A}^{0}(X)$ be the Lie subalgebra of the $A$-derivations of $X$ which send $X_{0}$ to $X_{0}$. Let $D$ be a Lie subalgebra of $\operatorname{Der}_{A}^{0}(X)$ and assume there is an $A$-bilinear transformation $\alpha: X_{0} \times X_{0} \rightarrow D$ which is skew-symmetric.

Suppose now that $\mathfrak{A}$ is another unital commutative associative algebra over $\mathbb{C}$ and $Y, Y_{0}, D^{\prime}$ are similarly defined for $\mathfrak{A}$. Assume $\beta: Y_{0} \times Y_{0} \rightarrow D^{\prime}$ is an $\mathfrak{A}$-bilinear transformation which is skew-symmetric. Suppose that

$$
\left[d, \alpha\left(x, x^{\prime}\right)\right]=\alpha\left(d x, x^{\prime}\right)+\alpha\left(x, d x^{\prime}\right) \quad \text { and } \quad\left[d^{\prime}, \beta\left(y, y^{\prime}\right)\right]=\beta\left(d^{\prime} y, y^{\prime}\right)+\beta\left(y, d^{\prime} y^{\prime}\right)
$$

for all $x, x^{\prime} \in X_{0}, y, y^{\prime} \in Y_{0}, d \in D$ and $d^{\prime} \in D^{\prime}$. Then the vector space

$$
\mathcal{T}(X / A, Y / \mathfrak{A}):=(D \otimes \mathfrak{A}) \oplus\left(X_{0} \otimes Y_{0}\right) \oplus\left(A \otimes D^{\prime}\right)
$$

is an algebra over $\mathbb{C}$ with the anticommutative multiplication given by

$$
\begin{aligned}
& {\left[d_{1} \otimes b, a \otimes d_{1}^{\prime}\right]=0, \quad\left[d_{1} \otimes b, d_{2} \otimes b^{\prime}\right]=\left[d_{1}, d_{2}\right] \otimes b b^{\prime},} \\
& {\left[a \otimes d_{1}^{\prime}, a^{\prime} \otimes d_{2}^{\prime}\right]=a a^{\prime} \otimes\left[d_{1}^{\prime}, d_{2}^{\prime}\right],} \\
& {\left[d_{1} \otimes b, x \otimes y\right]=d_{1} x \otimes b y=-\left[x \otimes y, d_{1} \otimes b\right],} \\
& {\left[a \otimes d_{1}^{\prime}, x \otimes y\right]=a x \otimes d_{1}^{\prime} y=-\left[x \otimes y, a \otimes d_{1}^{\prime}\right],} \\
& {\left[x \otimes y, x^{\prime} \otimes y^{\prime}\right]=\alpha\left(x, x^{\prime}\right) \otimes t\left(y, y^{\prime}\right)+\left(x * x^{\prime}\right) \otimes\left(y * y^{\prime}\right)+t\left(x, x^{\prime}\right) \otimes \beta\left(y, y^{\prime}\right)}
\end{aligned}
$$

for $d_{1}, d_{2} \in D, b, b^{\prime} \in \mathfrak{A}, a, a^{\prime} \in A, d_{1}^{\prime}, d_{2}^{\prime} \in D^{\prime}, x, x^{\prime} \in X_{0}$ and $y, y^{\prime} \in Y_{0}$. This is called a generalized Tits construction. If $X, Y$ are suitably chosen, then $\mathcal{T}(X / A, Y / \mathfrak{A})$ will be a Lie algebra $[\mathrm{BZ}$, Proposition 3.9].

2.1.2. Root graded Lie algebras. The main purpose of this work is to give a finite presentation for the universal covering algebra of a Lie torus. Lie tori are centerless cores of extended affine Lie algebras [Yos. In [AG] and [BGK], the authors classify the Lie tori under consideration, using the so called recognition theorems for root graded Lie algebras. We recall here these theorems and some related topics which will be of use in the proof of our main theorem. In what follows we denote the 8-dimensional octonion (Cayley) algebra by $\mathfrak{C}$. We consider the usual normalized trace on $\mathfrak{C}$ and denote by $\mathfrak{C}_{0}$ its subspace of trace zero elements. The 
algebra of inner derivations of $\mathfrak{C}$ is known to be a finite-dimensional simple Lie algebra of type $G_{2}$. Also we denote by $\mathfrak{J}$ the exceptional simple Jordan algebra whose inner derivations form a finite-dimensional simple algebra of type $F_{4}$. In fact if $\mathfrak{C}_{3 \times 3}$ is the algebra of $3 \times 3$ matrices with entries from the octonion algebra $\mathfrak{C}$, then $\mathfrak{J}$ is its subspace of self-adjoint elements, under transpose-conjugate involution $x \mapsto \bar{x}^{t}$, with the product $x \cdot y:=(x y+y x) / 2$. We also consider the usual normalized trace on $\mathcal{J}$ and denote the subspace of trace zero elements of $\mathfrak{J}$ by $\mathfrak{J}_{0}$.

Definition 2.1. Suppose that $\mathcal{G}$ is a finite-dimensional simple Lie algebra over $\mathbb{C}$ with a Cartan subalgebra $\mathcal{H}$ and root system $R$ so that $\mathcal{G}$ has a root space decomposition $\mathcal{G}=\bigoplus_{\mu \in R} \mathcal{G}_{\mu}$ with $\mathcal{H}=\mathcal{G}_{0}$. An $R$-graded Lie algebra $\mathcal{L}$ over $\mathbb{C}$ with grading pair $(\mathcal{G}, \mathcal{H})$ is a Lie algebra satisfying the following conditions:

(i) $\mathcal{L}$ contains $\mathcal{G}$ as a subalgebra,

(ii) $\mathcal{L}=\bigoplus_{\mu \in R} \mathcal{L}_{\mu}$, where $\mathcal{L}_{\mu}:=\{x \in \mathcal{L} \mid[h, x]=\mu(h) x$ for all $h \in \mathcal{H}\}$,

(iii) $\mathcal{L}_{0}=\sum_{\mu \in R^{\times}}\left[\mathcal{L}_{\mu}, \mathcal{L}_{-\mu}\right]$.

For a positive integer $\nu$, an $R$-graded Lie algebra $\mathcal{L}$ with grading pair $(\mathcal{G}, \mathcal{H})$ is called $\left(R, \mathbb{Z}^{\nu}\right)$-graded if $\mathcal{L}=\bigoplus_{\sigma \in \mathbb{Z}^{\nu}} \mathcal{L}^{\sigma}$ is a $\mathbb{Z}^{\nu}$-graded Lie algebra such that $\mathcal{G} \subseteq \mathcal{L}^{0}$ and $\operatorname{supp}(\mathcal{L}):=\left\{\sigma \in \mathbb{Z}^{\nu} \mid \mathcal{L}^{\sigma} \neq\{0\}\right\}$ generates $\mathbb{Z}^{\nu}$. Since $\mathcal{G} \subseteq \mathcal{L}^{0}, \mathcal{L}^{\sigma}$ is an $\mathcal{H}$-module for $\sigma \in \mathbb{Z}^{\nu}$ and so we have $\mathcal{L}=\bigoplus_{\mu \in R} \bigoplus_{\sigma \in \mathbb{Z}^{\nu}} \mathcal{L}_{\mu}^{\sigma}$ where $\mathcal{L}_{\mu}^{\sigma}:=\mathcal{L}^{\sigma} \cap \mathcal{L}_{\mu}$ for $\sigma \in \mathbb{Z}^{\nu}$ and $\mu \in R$. An $\left(R, \mathbb{Z}^{\nu}\right)$-graded Lie algebra $\mathcal{L}$ is called division $\left(R, \mathbb{Z}^{\nu}\right)$ graded if for each $\mu \in R^{\times}, \sigma \in \mathbb{Z}^{\nu}$ and $0 \neq x \in \mathcal{L}_{\mu}^{\sigma}$, there exists $y \in \mathcal{L}_{-\mu}^{-\sigma}$ such that modulo $Z(\mathcal{L}),[x, y]$ equals the unique element of $\mathcal{H}$ representing $\mu$ through the induced form on the dual of $\mathcal{H}$. A division $\left(R, \mathbb{Z}^{\nu}\right)$-graded Lie algebra $\mathcal{L}$ with $\operatorname{dim}_{\mathbb{C}}\left(\mathcal{L}_{\mu}^{\sigma}\right) \leq 1$ for all $\sigma \in \mathbb{Z}^{\nu}$ and $\mu \in R^{\times}$is called a Lie $\nu$-torus or simply a Lie torus. In this case the set $\left\{\alpha+\sigma \mid \alpha \in R, \sigma \in \mathbb{Z}^{\nu}, \mathcal{L}_{\alpha}^{\sigma} \neq\{0\}\right\} \subseteq \operatorname{span}_{\mathbb{R}} R \oplus \mathbb{Z}^{\nu}$ is called the root system of $\mathcal{L}$. For $\mu \in R^{\times}$, define $S_{\mu}:=\left\{\sigma \in \mathbb{Z}^{\nu} \mid \mathcal{L}_{\mu}^{\sigma} \neq\{0\}\right\}$; by Yos, Theorem 1.5], $S_{\mu}=S_{\nu}$ if $\mu$ and $\nu$ have the same length. If two root lengths occur, we set $S:=S_{\mu}$ for any choice of a short root $\mu$ and $L:=S_{\nu}$ for any choice of a long root $\nu$ and call $(S, L)$ the corresponding pair of $\mathcal{L}$.

Definition 2.2. Let $B$ be a unital commutative associative algebra, $\mathcal{W}$ be a $B$ module and $g: \mathcal{W} \times \mathcal{W} \rightarrow B$ be a symmetric $B$-bilinear form on $\mathcal{W}$. Then $J(\mathcal{W}):=$ $B 1 \oplus \mathcal{W}$ with the multiplication, for $w, w^{\prime} \in \mathcal{W}, b, b^{\prime} \in B$,

$$
(b 1+w) \cdot\left(b^{\prime} 1+w^{\prime}\right)=b b^{\prime} 1+g\left(w, w^{\prime}\right) 1+b w^{\prime}+b^{\prime} w
$$

is a Jordan algebra called the Clifford Jordan algebra of $g$.

Definition 2.3. Two perfect Lie algebras are said to be centrally isogenous if they have the same universal covering algebra, up to isomorphism. 
Theorem 2.1 (Recognition Theorem). Let $\mathcal{L}$ be an R-graded Lie algebra with grading pair $(\mathcal{G}, \mathcal{H})$.

(i) ([BM]) If $R$ is simply laced, there is a commutative associative unital algebra $A$ such that $\mathcal{L}$ is centrally isogenous with $\mathcal{G} \otimes A$.

(ii) ([BZ]) If $R$ is of type $B_{\ell}, \ell \geq 3$, there exists a unital commutative associative algebra $A$ and a unital $A$-module $B$ with a symmetric $A$-bilinear form $(\cdot, \cdot)$ : $B \times B \rightarrow A$ such that $\mathcal{L}$ is centrally isogenous with

$$
\mathcal{T}(J(\mathcal{V}) / \mathbb{C}, J(B) / A)=(\mathcal{G} \otimes A) \oplus(\mathcal{V} \otimes B) \oplus D_{J(B), J(B)}
$$

where $\mathcal{V}$ is the $(2 \ell+1)$-dimensional vector space equipped with a non-degenerate symmetric bilinear form with respect to which the set of skew-symmetric endomorphisms of $\mathcal{V}$ is isomorphic to $\mathcal{G}$.

(iii) ([BZ] If $R$ is of type $G_{2}$, then there is a unital commutative associative algebra $A$ and a unital Jordan algebra $J$ over $A$ having a normalized trace $T$ satisfying the identity

$\operatorname{ch}_{3}(y):=y^{3}-3 T(y) y^{2}+\left(\frac{9}{2} T(y)^{2}-\frac{3}{2} T\left(y^{2}\right)\right) y+\left(T\left(y^{3}\right)-\frac{9}{2} T\left(y^{2}\right) T(y)+\frac{9}{2} T(y)^{3}\right) 1=0$ such that $\mathcal{L}$ is centrally isogenous with

$$
\mathcal{T}(\mathfrak{C} / \mathbb{C}, J / A)=(\mathcal{G} \otimes A) \oplus\left(\mathfrak{C}_{0} \otimes J_{0}\right) \oplus D_{J, J}
$$

where $\mathfrak{C}_{0}$ and $J_{0}$ are the trace zero elements of the octonion algebra $\mathfrak{C}$ and $J$ respectively.

(iv) ([BZ] If $R$ is of type $F_{4}$, there exists a unital commutative associative algebra $A$ and a unital alternative algebra $\mathcal{A}$ over $A$ having a normalized trace $T$ satisfying the identity

$$
\operatorname{ch}_{2}(y):=y^{2}-2 T(y) y+\left(2 T(y)^{2}-T\left(y^{2}\right)\right) 1=0
$$

such that $\mathcal{L}$ is centrally isogenous with

$$
\mathcal{T}(\mathfrak{J} / \mathbb{C}, \mathcal{A} / A)=(\mathcal{G} \otimes A) \oplus\left(\mathfrak{J}_{0} \otimes \mathcal{A}_{0}\right) \oplus D_{\mathcal{A}, \mathcal{A}},
$$

where $\mathcal{A}_{0}$ is the subspace of trace zero elements of $\mathcal{A}$.

Let $\mathcal{L}$ be any $R$-graded Lie algebra. By the Recognition Theorem (and its proof), we know that $\mathcal{L}$ has a decomposition as

$$
(\mathcal{G} \otimes A) \oplus(\mathcal{V} \otimes B) \oplus \mathcal{D}
$$

so that $\mathcal{G} \otimes 1$ is identified with $\mathcal{G}$ of Definition 2.1(i), where $\mathcal{V}$ is the irreducible finitedimensional $\mathcal{G}$-module whose highest weight is the highest short root, equipped 
with a normalized trace, and $\mathcal{D}$ is a subalgebra of $\mathcal{L}$. Also $A, B$ are two vector spaces, with $B=0$ in simply laced cases, such that $\mathfrak{a}:=A \oplus B$ is an algebra which we refer to as the coordinate algebra of $\mathcal{L}$ (see $[\overline{\mathrm{BGKN}}],[\mathrm{BM}]$ ). It is noticeable that using (2.4), one can see that $Z(\mathcal{L}) \subseteq \mathcal{D}$, and $\mathcal{L} / Z(\mathcal{L})$ is centerless as $\mathcal{L}$ is perfect.

Theorem 2.2 (验, Theorem 4.13]). Let $\mathcal{L}=(\mathcal{G} \otimes A) \oplus(\mathcal{V} \otimes B) \oplus D_{\mathfrak{a}, \mathfrak{a}}$ be a centerless $R$-graded Lie algebra where $\mathfrak{a}=A \oplus B$ is the coordinate algebra of $\mathcal{L}$. Take $\mathfrak{s}$ to be the subspace of $\mathfrak{a} \otimes \mathfrak{a}$ spanned by the elements of the form

$$
(\alpha \otimes \beta)+(\beta \otimes \alpha), \quad(\alpha \beta \otimes \gamma)+(\beta \gamma \otimes \alpha)+(\gamma \alpha \otimes \beta), \quad a \otimes b
$$

for $a \in A, b \in B, \alpha, \beta, \gamma \in \mathfrak{a}$. Consider the factor space

$$
\{\mathfrak{a}, \mathfrak{a}\}:=(\mathfrak{a} \otimes \mathfrak{a}) / \mathfrak{s}
$$

and for $\alpha, \beta \in \mathfrak{a}$, let $\{\alpha, \beta\}$ denote $(\alpha \otimes \beta)+\mathfrak{s}$ in $\{\mathfrak{a}, \mathfrak{a}\}$. Set $\hat{\mathcal{L}}:=(\mathcal{G} \otimes A) \oplus(\mathcal{V} \otimes$ $B) \oplus\{\mathfrak{a}, \mathfrak{a}\}$. Define a multiplication on $\hat{\mathcal{L}}$ by

$$
\begin{aligned}
& {\left[x \otimes a, x^{\prime} \otimes a^{\prime}\right]=\left[x, x^{\prime}\right] \otimes a a^{\prime}+\kappa\left(x, x^{\prime}\right)\left\{a, a^{\prime}\right\},} \\
& {[x \otimes a, v \otimes b]=x v \otimes a b=-[v \otimes b, x \otimes a],} \\
& {\left[x \otimes a,\left\{\alpha, \alpha^{\prime}\right\}\right]=0=-\left[\left\{\alpha, \alpha^{\prime}\right\}, x \otimes a\right],} \\
& {\left[v \otimes b, v^{\prime} \otimes b^{\prime}\right]=D_{v, v^{\prime}} \otimes t\left(b, b^{\prime}\right)+\left(v * v^{\prime}\right) \otimes\left(b * b^{\prime}\right)+t\left(v, v^{\prime}\right)\left\{b, b^{\prime}\right\},} \\
& {\left[\left\{\alpha, \alpha^{\prime}\right\}, v \otimes b\right]=v \otimes D_{\alpha, \alpha^{\prime}} b=-\left[v \otimes b,\left\{\alpha, \alpha^{\prime}\right\}\right],} \\
& {\left[\left\{\alpha, \alpha^{\prime}\right\},\left\{\beta, \beta^{\prime}\right\}\right]=\left\{D_{\alpha, \alpha^{\prime}} \beta, \beta^{\prime}\right\}+\left\{\beta, D_{\alpha, \alpha^{\prime}} \beta^{\prime}\right\},}
\end{aligned}
$$

for $x, x^{\prime} \in \mathcal{G}, a, a^{\prime} \in A, v, v^{\prime} \in \mathcal{V}, b, b^{\prime} \in B$ and $\alpha, \alpha^{\prime}, \beta, \beta^{\prime} \in \mathfrak{a}$ where $\kappa$ denotes the Killing form of $\mathcal{G}$. Also consider the map $\hat{\pi}: \hat{\mathcal{L}} \rightarrow \mathcal{L}$ given by $x \otimes a \mapsto x \otimes a ; u \otimes b \mapsto$ $u \otimes b ;\left\{\alpha, \alpha^{\prime}\right\} \mapsto D_{\alpha, \alpha^{\prime}}$. Then $(\hat{\mathcal{L}}, \hat{\pi})$ is the universal covering algebra of $\mathcal{L}$.

To state the next theorem, we need to recall some algebras from [BGKN] and AABGP. For $1 \leq p \leq 3$ with $p \leq \nu$, take $\mathcal{A}_{0}$ to be $A_{[\nu]}$ and

$$
\mathcal{A}_{p}:=\mathcal{A}_{p-1} \oplus \mathcal{A}_{p-1} x_{p}
$$

to be the algebra obtained from $\mathcal{A}_{p-1}$ using the Cayley-Dickson process with

$$
x_{p}^{2}=t_{p}
$$

The last one is called the Cayley torus (or octonion torus). We mention that the Cayley torus is alternative but not associative; the center and the nucleus of this algebra coincide with $A_{[\nu]}$. We know that $\mathcal{A}_{p-1}$ is a subalgebra of $\mathcal{A}_{p}$ for $1 \leq p \leq 3$. Moreover these four algebras are algebras over $A_{[\nu]}$, in fact they are free $A_{[\nu]}$-modules. Next note that for $1 \leq i \leq 8$, there exist unique $s_{1}, s_{2}, s_{3} \in\{0,1\}$ 
such that $i=1+s_{1}+2 s_{2}+4 s_{3}$. Take $w_{i}:=\left(x_{1}^{s_{1}} x_{2}^{s_{2}}\right) x_{3}^{s_{3}}$. Then $\left\{w_{i} \mid 1 \leq i \leq 2^{p}\right\}$ is an $A_{[\nu]}$-basis for $\mathcal{A}_{p}, 0 \leq p \leq 3$. We consider the normalized trace

$$
T: \mathcal{A}_{p} \rightarrow A_{[\nu]} ; \sum_{i=1}^{2^{p}} r_{i} w_{i} \mapsto r_{1},
$$

on $\mathcal{A}_{p}, 0 \leq p \leq 3$, and define $t$ and $*$ to be as before.

Next take $\mathcal{J}_{0}:=A_{[\nu]}, \mathcal{J}_{1}$ to be the commutative associative algebra over $A_{[\nu]}$ with generator $x_{1}$ subject to the relation $x_{1}^{3}=t_{1}$, and $\mathcal{J}_{2}$ to be the plus algebra of the associative algebra over $A_{[\nu]}$ generated by $x_{1}, x_{2}$ subject to the relations $x_{1}^{3}=t_{1}, x_{2}^{3}=t_{2}, x_{1} x_{2}=e^{2 \pi i / 3} x_{2} x_{1}$. Let $\mathcal{J}_{3}:=\mathcal{J}_{2} \oplus\left(\mathcal{J}_{2} \cdot x_{3}\right) \oplus\left(\mathcal{J}_{2} \cdot x_{3}^{2}\right)$ be the Jordan algebra obtained from $\mathcal{J}_{2}$ using Tits' first Jordan algebra construction [J], Chapter IX] with $x_{3}^{3}=t_{3}$. We mention that whenever we use $\mathcal{J}_{p}, 1 \leq p \leq 3$, we assume $p \leq \nu$.

One sees that for $1 \leq i \leq 27$, there exist unique $s_{1}, s_{2}, s_{3} \in\{0,1,2\}$ such that $i=1+s_{1}+3 s_{2}+9 s_{3}$. Take $w_{i}:=\left(x_{1}^{s_{1}} \cdot x_{2}^{s_{2}}\right) \cdot x_{3}^{s_{3}}$. Then $\left\{w_{i} \mid 1 \leq i \leq 3^{p}\right\}$ is an $A_{[\nu]}$-basis for $\mathcal{J}_{p}, 0 \leq p \leq 3$. Define the following normalized trace on $\mathcal{J}_{p}$, $0 \leq p \leq 3$ :

$$
T: \mathcal{J}_{p} \rightarrow A_{[\nu]} ; \sum_{i=1}^{3^{p}} r_{i} w_{i} \mapsto r_{1}
$$

Theorem 2.3. Let $\mathcal{L}$ be the centerless core of an extended affine Lie algebra of type $R$. Taking $\mathcal{G}$ to be a finite-dimensional simple Lie algebra of type $R, \mathcal{L}$ is isomorphic to one of the following:

(i) $([\mathrm{BGK}]) \mathcal{G} \otimes A_{[\nu]}$ if $R$ is simply laced.

(ii) $([\mathrm{AG}]) \mathcal{T}\left(J(\mathcal{V}) / \mathbb{C}, J\left(A_{[\nu]}^{m}\right) / A_{[\nu]}\right)$ if $R$ is of type $B_{\ell}(\ell \geq 3)$, where $\mathcal{V}$ is the $2 \ell+1$-dimensional vector space having a symmetric non-degenerate bilinear form with respect to which the set of skew-symmetric endomorphisms of $\mathcal{V}$ is isomorphic to $\mathcal{G}$ and $J\left(A_{[\nu]}^{m}\right)$ is the Clifford Jordan algebra with respect to the symmetric $A_{[\nu]}$-bilinear form on $A_{[\nu]}^{m}$ given by

$$
\begin{gathered}
g: A_{[\nu]}^{m} \times A_{[\nu]}^{m} \rightarrow A_{[\nu]}, \\
g\left(\sum_{r=1}^{m} a_{r} w_{r}, \sum_{r=1}^{m} b_{r} w_{r}\right)=\sum_{r=1}^{m} a_{r} b_{r} t^{\tau_{r}}
\end{gathered}
$$

where $\tau_{0}, \ldots, \tau_{m} \in \mathbb{Z}^{\nu}$ satisfy $\tau_{0}=0$ and $\tau_{r} \neq \equiv \tau_{s}\left(\bmod 2 \mathbb{Z}^{\nu}\right)$ for $0 \leq s \neq r \leq m$. Moreover the root system of $\mathcal{L}$ is of the form $(S+S) \cup\left(R_{\mathrm{sh}}+S\right) \cup\left(R_{\mathrm{lg}}+2 \mathbb{Z}^{\nu}\right)$ where $S=\bigcup_{j=0}^{m}\left(2 \mathbb{Z}^{\nu}+\tau_{j}\right)$. (Here $\left\{w_{1}, \ldots, w_{m}\right\}$ is the standard basis of $A_{[\nu]}^{m}$ as a free $A_{[\nu]}$-module.) 
(iii) ([AG]) $\mathcal{T}\left(\mathfrak{J} / \mathbb{C}, \mathcal{C} / A_{[\nu]}\right)$ if $R$ is of type $F_{4}$, where $\mathcal{C}=\mathcal{A}_{p}$ for some $0 \leq p \leq 3$. Moreover, if $\mathcal{C}=\mathcal{A}_{p}, 0 \leq p \leq 3$, then the root system of $\mathcal{L}$ is $\mathbb{Z}^{\nu} \cup\left(R_{\mathrm{sh}}+\mathbb{Z}^{\nu}\right) \cup$ $\left(R_{\lg }+\left(2 \mathbb{Z}^{p} \oplus \mathbb{Z}^{\nu-p}\right)\right)$.

(iv) ([AG]) $\mathcal{T}\left(\mathfrak{C} / \mathbb{C}, \mathcal{J} / A_{[\nu]}\right)$ if $R$ is of type $G_{2}$, where $\mathcal{J}=\mathcal{J}_{p}$ for some $0 \leq p \leq 3$. Moreover, if $\mathcal{J}=\mathcal{J}_{p}, 0 \leq p \leq 3$, then the root system of $\mathcal{L}$ is $\mathbb{Z}^{\nu} \cup\left(R_{\mathrm{sh}}+\mathbb{Z}^{\nu}\right) \cup$ $\left(R_{\lg }+\left(3 \mathbb{Z}^{p} \oplus \mathbb{Z}^{\nu-p}\right)\right)$.

Remark. Using the same notation as before, if $\mathfrak{a}=A \oplus B$ is the coordinate algebra of a Lie torus $\mathcal{L}$ with the universal covering algebra $\mathfrak{A}$, one sees from $\mathrm{BZ}$ that $A$ is a subset of the associative center of $\mathfrak{a}$ and so 2.5 implies that $\{A, A\}=\left\{\left\{a, a^{\prime}\right\} \mid\right.$ $\left.a, a^{\prime} \in A\right\} \subseteq Z(\mathfrak{A})$.

\section{$\S 2.2$. Induced relations}

As before we assume that $R$ is a finite irreducible reduced root system of type $X \neq A, C$. Let $\mathcal{L}$ be a centerless Lie $\nu$-torus of type $R$ and $\mathfrak{A}$ be its universal covering algebra. Using [Yos, Theorem 7.3], we identify $\mathcal{L}$ with the centerless core of an extended affine Lie algebra and so by Theorems 2.3 and 2.2 , we may write

$$
\mathfrak{A}=\left(\mathcal{G} \otimes A_{[\nu]}\right) \oplus\left(\mathcal{V} \otimes A_{[\nu]}^{m}\right) \oplus \mathcal{D}
$$

for some non-negative integer $m$, where $\mathcal{G}$ is a finite-dimensional simple Lie algebra of type $X, \mathcal{V}$ is an irreducible $\mathcal{G}$-module whose highest weight is the highest short root of $\mathcal{G}$, and $\mathcal{D}$ is a known subalgebra of $\mathfrak{A}$. We remark that if $X$ is simply laced, we have by convention $m=0$ and so the middle part in 2.90 vanishes. Since the ingredients appearing in (2.9) and their algebra structures are completely known (see $[\mathrm{BGK}, \overline{\mathrm{AG}}$ and $[\mathrm{ABG}]$ ), we are able to select a set of generators for $\mathfrak{A}$ and deduce certain relations among them which in turn motivate the generators and defining relations of a presented Lie algebra (isomorphic to $\mathfrak{A}$ ) which we define in the next section.

If $\mathfrak{A}=\sum_{\alpha \in R} \mathfrak{A}_{\alpha}, \mathcal{G}=\sum_{\alpha \in R} \mathcal{G}_{\alpha}$ and $\mathcal{V}=\left(\sum_{\alpha \in R_{\mathrm{sh}}} \mathcal{V}_{\alpha}\right) \oplus \mathcal{V}_{0}$, are the corresponding weight space decompositions of $\mathfrak{A}, \mathcal{G}$ and $\mathcal{V}$ respectively, then

$$
\mathfrak{A}_{\alpha}= \begin{cases}\mathcal{G}_{\alpha} \otimes A_{[\nu]} & \text { if } \alpha \in R_{\mathrm{lg}}, \\ \left(\mathcal{G}_{\alpha} \otimes A_{[\nu]}\right) \oplus\left(\mathcal{V}_{\alpha} \otimes A_{[\nu]}^{m}\right) & \text { if } \alpha \in R_{\mathrm{sh}}, \\ \sum_{\beta \in R^{\times}}\left[\mathfrak{A}_{\beta}, \mathfrak{A}_{-\beta}\right] & \text { if } \alpha=0 .\end{cases}
$$

It is known that if $X$ is not simply laced, then $\mathcal{G}$ is the set of inner derivations of an algebra equipped with a normalized trace $T$, and that $\mathcal{V}$ is the set of zeros of this trace. We fix a highest weight vector $\mathbf{v}:=v_{n_{s}}$ of $\mathcal{V}$. Next using Proposition 2.2(i), we fix $j_{1}^{t}, \ldots, j_{n_{t}}^{t}, k_{1}^{t}, \ldots, k_{n_{t}^{\prime}}^{t} \in J_{\ell}, n_{\ell}+1 \leq t \leq n_{s}$, such that

$$
v_{t}:=\left[f_{j_{1}^{t}}, \ldots, f_{j_{n_{t}}^{t}}, \mathbf{v}\right] \quad \text { and } \quad v_{-t}=\left[f_{k_{1}^{t}}, \ldots, f_{k_{n_{t}^{t}}^{t}}, \mathbf{v}\right]
$$


are non-zero elements of $\mathcal{V}_{\alpha_{t}}$ and $\mathcal{V}_{-\alpha_{t}}$ respectively. Then

$$
\mathcal{V}=\left(\bigoplus_{t=n_{\ell}+1}^{n_{s}} \mathbb{C} v_{ \pm t}\right) \oplus \mathcal{V}_{0}
$$

where $\mathcal{V}_{0}$ is the corresponding zero weight space. We recall the trace form $t$ as in (2.3) and ask the reader to check that

$$
t\left(v_{n_{s}}, v_{ \pm i}\right)=0 \quad\left(n_{\ell}+1 \leq i \leq n_{s}-1\right) .
$$

Now consider the coordinate algebra $\mathfrak{a}=A_{[\nu]} \oplus A_{[\nu]}^{m}$ of $\mathcal{L}$. Using Theorem 2.1 we find that $A_{[\nu]}^{m}$ is the kernel of a normalized trace $T$ of $\mathfrak{a}$. We recall the trace form $t(\cdot, \cdot): \mathfrak{a} \times \mathfrak{a} \rightarrow A_{[\nu]}, t(x, y):=T(x y)$, and the operator $*: \mathfrak{a} \times \mathfrak{a} \rightarrow A_{[\nu]}^{m}$ defined by $x * y:=x y-t(x, y) 1$. Let $\left\{w_{r} \mid 1 \leq r \leq m\right\}$ be the standard basis for the $A_{[\nu]}$-module $A_{[\nu]}^{m}$. Then one observes that for $1 \leq r, s \leq m$, there are unique constants $a_{r, s}, a_{r, s}^{\prime} \in \mathbb{C}, t_{r, s} \in J_{m}$ and unique $\nu$-tuples $\sigma_{r, s}, \sigma_{r, s}^{\prime} \in \mathbb{Z}^{\nu}$ with

$$
\begin{aligned}
& \sigma_{r, s}=\sigma_{s, r}, t_{r, s}=t_{s, r}, a_{r, s}=-a_{s, r} \quad \text { for type } F_{4}, \\
& a_{r, s}=a_{s, r} \quad \text { for type } B, G_{2}, \\
& \left(1-\delta_{r, s}\right) a_{r, s}^{\prime}+\delta_{r, s} a_{r, s}=0 \text { and }\left(1-\delta_{r, s}\right) a_{r, s}+\delta_{r, s} a_{r, s}^{\prime} \neq 0
\end{aligned}
$$

satisfying

$$
t\left(w_{r}, w_{s}\right)=a_{r, s}^{\prime} t^{\sigma_{r, s}^{\prime}} \quad \text { and } \quad w_{r} * w_{s}=a_{r, s} t^{\sigma_{r, s}} w_{t_{r, s}}
$$

We set

$$
\mathfrak{D}_{c}:=\left\{a_{r, s}, a_{r, s}^{\prime}, \sigma_{r, s}, \sigma_{r, s}^{\prime}, t_{r, s} \mid r, s \in J_{m}\right\}
$$

We consider $\mathfrak{D}_{c}$ as a data-set for the coordinate algebra $\mathfrak{a}$ of $\mathcal{L}$. In fact, as $\left\{w_{i} \mid\right.$ $\left.i \in J_{m}\right\}$ is an $A_{[\nu]}$-basis for $A_{[\nu]}^{m}$, and also the *-operator and $t(\cdot, \cdot)$ are $A_{[\nu]}$-bilinear, the data in $\mathfrak{D}_{c}$ completely describe the structure of $\mathfrak{a}=A_{[\nu]} \oplus A_{[\nu]}^{m}$.

Next for $n_{\ell}+1 \leq i \leq n_{s}-1$, define $t_{ \pm i}^{\prime} \in J_{n}$ and $n_{\ell}+1 \leq t_{i} \leq n_{s}$ as follows. If $\alpha_{n_{s}} \pm \alpha_{i}$ is a root, take $t_{ \pm i}^{\prime}$ to be such that $\alpha_{n_{s}} \pm \alpha_{i}=\alpha_{t_{ \pm i}^{\prime}}$, and $n$ otherwise. Also if $\alpha_{n_{s}}-\alpha_{i}$ is a short root, take $t_{i}$ to be such that $\alpha_{n_{s}}-\alpha_{i}=\alpha_{t_{i}}$, and $n_{s}$ otherwise. One observes that there are $m_{ \pm i}, m_{ \pm i}^{\prime} \in \mathbb{C}$ with $m_{ \pm i}^{\prime}=0$ and $m_{ \pm i}=0$ if $\alpha_{n_{s}} \pm \alpha_{i}$ is not a root or a short root respectively such that

$$
d_{v_{n_{s}}, v_{ \pm i}}=m_{ \pm i}^{\prime} e_{t_{ \pm i}^{\prime}} \quad \text { and } \quad v_{n_{s}} * v_{ \pm i}=m_{ \pm i} v_{t_{i}} .
$$

We set

$$
\mathfrak{D}_{m}:=\left\{t_{ \pm i}^{\prime}, t_{i}, m_{ \pm i}, m_{ \pm i}^{\prime} \mid n_{\ell}+1 \leq i \leq n_{s}-1\right\}
$$

We draw the attention of the reader to the fact that the data appearing in $\mathfrak{D}_{m}$ are derived from the structure of the $\mathcal{G}$-module $\mathcal{V}$. We next take $C$ to be the Cartan 
matrix of $R$ with respect to $\Delta$ and call

$$
\mathfrak{D}:=\left(C, \mathfrak{D}_{m}, \mathfrak{D}_{c}\right)
$$

the structural data associated to $\mathcal{L}$.

Now let $x_{1}, \ldots, x_{p} \in \mathcal{H}$ and $y_{1}, \ldots, y_{q}$ be some non-zero root vectors of $\mathcal{G}$. Let $j_{1}, \ldots, j_{p} \in J_{\ell}$ and set $t^{\sigma}=t_{j_{1}}^{ \pm 1} \cdots t_{j_{p}}^{ \pm 1}$. Then it follows from 2.5 that, for $1 \leq i \leq m$

$$
\begin{aligned}
& {\left[y_{1} \otimes 1, \ldots, y_{q} \otimes 1, x_{1} \otimes t_{j_{1}}^{ \pm 1}, \ldots, x_{p} \otimes t_{j_{p}}^{ \pm 1}, e_{n} \otimes 1\right]} \\
& =\left[y_{1}, \ldots, y_{q}, x_{1}, \ldots, x_{p}, e_{n}\right] \otimes t^{\sigma} \\
& {\left[y_{1} \otimes 1, \ldots, y_{q} \otimes 1, x_{1} \otimes t_{j_{1}}^{ \pm 1}, \ldots, x_{p} \otimes t_{j_{p}}^{ \pm 1}, v_{n_{s}} \otimes w_{i}\right]} \\
& =y_{1} \ldots y_{q} x_{1} \ldots x_{p} v_{n_{s}} \otimes t^{\sigma} w_{i}
\end{aligned}
$$

Next suppose that $\left\{e_{i}, f_{i}, h_{i} \mid i \in J_{\ell}\right\}$ is a set of Chevalley generators for $\mathcal{G}$. We claim that

$$
\left\{e_{i} \otimes 1, f_{i} \otimes 1, h_{i} \otimes 1, h_{i} \otimes t_{j}^{ \pm 1}, \mathbf{v} \otimes w_{t} \mid i \in J_{\ell}, j \in J_{\nu}, t \in J_{m}\right\}
$$

is a generating set for $\mathfrak{A}$ (recall that $\mathbf{v}=v_{n_{s}}$ ). Indeed, using 2.10 , it is enough to show that $\left(\mathcal{G} \otimes A_{[\nu]}\right) \oplus\left(\mathcal{V} \otimes A_{[\nu]}^{m}\right)$ is generated by this set. We show $\mathcal{V} \otimes A_{[\nu]}^{m}$ is generated by the set 2.18; ; a similar argument works for $\mathcal{G} \otimes A_{[\nu]}$. Consider a generating element $v \otimes t^{\sigma} w_{i}$ of the vector space $\mathcal{V} \otimes A_{[\nu]}^{m}$ where $i \in J_{m}$ and $\sigma \in \mathbb{Z}^{\nu}$ with $t^{\sigma}=t_{j_{1}}^{ \pm 1} \cdots t_{j_{p}}^{ \pm 1}$ for some $j_{1}, \ldots, j_{p} \in J_{\nu}$. It is known that $v$ can be written as a linear combination of elements of the form $f_{i_{1}} \ldots f_{i_{q}} v_{n_{s}}$ for some $i_{1}, \ldots, i_{q} \in J_{\ell}$. So without loss of generality, we assume $i_{1}, \ldots, i_{q} \in J_{\ell}$ and $v=f_{i_{1}} \ldots f_{i_{q}} v_{n_{s}}$. Next we take $x_{1}, \ldots, x_{p} \in \mathcal{H}$ such that $\alpha_{n_{s}}\left(x_{1}\right) \neq 0, \ldots, \alpha_{n_{s}}\left(x_{p}\right) \neq 0$, so there is $r \in \mathbb{C} \backslash\{0\}$ such that $v_{n_{s}}=r x_{1} \ldots x_{p} v_{n_{s}}$. So by (2.17), we have

$$
\begin{aligned}
v \otimes t^{\sigma} w_{i} & =f_{i_{1}} \ldots f_{i_{q}} v_{n_{s}} \otimes t^{\sigma} w_{i}=r f_{i_{1}} \ldots f_{i_{q}} x_{1} \ldots x_{p} v_{n_{s}} \otimes t^{\sigma} w_{i} \\
& =r\left[f_{i_{1}} \otimes 1, \ldots, f_{i_{q}} \otimes 1, x_{1} \otimes t_{j_{1}}^{ \pm 1}, \ldots, x_{p} \otimes t_{j_{p}}^{ \pm 1}, v_{n_{s}} \otimes w_{i}\right]
\end{aligned}
$$

This proves the claim.

For $n_{\ell}+1 \leq i \leq n_{s}-1$ and $r, s \in J_{m}$, take $\sigma_{r, s}, \sigma_{r, s}^{\prime} \in \mathbb{Z}^{\nu}, m_{ \pm i}, m_{ \pm i}^{\prime}, a_{r, s}, a_{r, s}^{\prime} \in$ $\mathbb{C}, t_{ \pm i}^{\prime} \in J_{n}, t_{r, s} \in J_{m}, n_{\ell}+1 \leq t_{i} \leq n_{s}$ and $m_{ \pm i}, m_{ \pm i}^{\prime} \in \mathbb{C}$ as in 2.14 and 2.15). Using 2.5 and 2.12, one sees that

$$
\left[v_{n_{s}} \otimes w_{r}, v_{ \pm i} \otimes w_{s}\right]=\left(m_{ \pm i}^{\prime} a_{r, s}^{\prime} e_{t_{ \pm i}^{\prime}} \otimes t^{\sigma_{r, s}^{\prime}}\right)+\left(m_{ \pm i} a_{r, s} v_{t_{i}} \otimes t^{\sigma_{r, s}} w_{t_{r, s}}\right) .
$$

Following [You, we call this kind of relations basic short part relations. 
Next let $\alpha \in R^{\times}, \beta \in R_{\mathrm{sh}}, x_{\alpha} \in \mathcal{G}_{\alpha}$ and $v_{\beta} \in \mathcal{V}_{\beta}$. Then 2.5 implies that for $i, r \in J_{\ell}, j \in J_{\nu}$ and $s \in J_{m}$, the following relations are satisfied in $\mathfrak{A}$, the universal covering algebra of $\mathcal{L}$ :

$$
\begin{aligned}
& {\left[\alpha\left(h_{r}\right)\left(h_{i} \otimes t_{j}^{ \pm 1}\right)-\alpha\left(h_{i}\right)\left(h_{r} \otimes t_{j}^{ \pm 1}\right), x_{\alpha} \otimes 1\right]=0,} \\
& {\left[\beta\left(h_{r}\right)\left(h_{i} \otimes t_{j}^{ \pm 1}\right)-\beta\left(h_{i}\right)\left(h_{r} \otimes t_{j}^{ \pm 1}\right), v_{\beta} \otimes w_{s}\right]=0,}
\end{aligned}
$$

to which we refer as quasi-diagonal relations, and also

$$
\begin{aligned}
& {\left[h_{r} \otimes t_{j}, h_{i} \otimes t_{j}^{-1}, x \otimes 1\right]=\alpha\left(h_{i}\right) \alpha\left(h_{r}\right) x \otimes 1,} \\
& {\left[h_{r} \otimes t_{j}, h_{i} \otimes t_{j}^{-1}, y \otimes w_{s}\right]=\beta\left(h_{i}\right) \beta\left(h_{r}\right) y \otimes w_{s},}
\end{aligned}
$$

that we refer to as canceling relations.

\section{$\S 3 . \quad$ A generic presentation}

From now on we fix a centerless Lie torus $\mathcal{L}$ of type $X \neq A, C, B C$ and we let $\mathfrak{A}$ be its universal covering algebra. Let $\mathfrak{D}$ be the structural data associated to $\mathcal{L}$. In this section, starting from $\mathfrak{D}$, we introduce a presented Lie algebra $\tilde{\mathcal{L}}:=\tilde{\mathcal{L}}(\mathfrak{D})$, called the presented Lie algebra associated to $\mathcal{L}$ (or $\mathfrak{D}$ ). The main objective of this work is to show that $\tilde{\mathcal{L}}$ is isomorphic to $\mathfrak{A}$.

We use the same notation as in Section 2 In particular, we let $\mathcal{G}$ be the finite-dimensional simple Lie algebra of rank $\ell$ corresponding to the Cartan matrix $C=\left(c_{i, j}\right)$. Let $\mathcal{H}$ be a fixed Cartan subalgebra of $\mathcal{G}$ and $(\cdot, \cdot)$ be the Killing form of $\mathcal{G}$. We recall that $R$ is the root system of $\mathcal{G}$ and $\Delta$ is a base of $R$. Also $\mathcal{V}$ is an irreducible $\mathcal{G}$-module whose highest weight is the highest short root. We keep the same arrangement for roots as in (2.1). Throughout this work, by $\langle\alpha, \beta\rangle$, for roots $\alpha, \beta \in R$ with $\beta \neq 0$, we mean $2(\alpha, \beta) /(\beta, \beta)$. Let $m$ be a non-negative integer which we take to be zero if $R$ is simply laced. Whenever we use expressions containing a letter with subscripts going through $\{1, \ldots, m\}$, we understand that we are in the case $m \neq 0$. Now for a non-negative integer $\nu$, let $\tilde{\mathcal{L}}$ be the Lie algebra defined by $3 \ell+m+\ell \nu$ generators

$$
\left\{e_{i}, f_{i}, h_{i}, h_{i, a}^{ \pm}, v^{r} \mid i \in J_{\ell}, r \in J_{m}, a \in J_{\nu}\right\},
$$

subject to the following relations (we collect our relations, depending on their nature, in groups (R1)-(R9) below and give a name to some of these groups, based on the role which they play):

\section{Serre's relations:}

$$
\begin{aligned}
& {\left[h_{i}, h_{j}\right]=0, \quad\left[e_{i}, f_{j}\right]=\delta_{i, j} h_{i}, \quad\left[h_{i}, e_{j}\right]=c_{j, i} e_{j}, \quad\left[h_{i}, f_{j}\right]=-c_{j, i} f_{j},} \\
& \left(\operatorname{ad}_{i}\right)^{-c_{j, i}+1}\left(e_{j}\right)=0, \quad\left(\operatorname{ad} f_{i}\right)^{-c_{j, i}+1}\left(f_{j}\right)=0 ; \quad i, j \in J_{\ell} .
\end{aligned}
$$




\section{Highest short weight relations:}

$$
\begin{aligned}
& {\left[e_{i}, v^{r}\right]=0, \quad\left[h_{i}, v^{r}\right]=\left\langle\alpha_{n_{s}}, \alpha_{i}\right\rangle v^{r},} \\
& {\left[f_{i}^{\left\langle\alpha_{n_{s}}, \alpha_{i}\right\rangle+1}, v^{r}\right]=0 ; \quad i \in J_{\ell}, r \in j_{m} .}
\end{aligned}
$$

Since $\left\{e_{i}, f_{i}, h_{i}\right\}_{i=1}^{\ell}$ satisfies Serre's relations, the subalgebra of $\tilde{\mathcal{L}}$ generated by the $3 \ell$ elements $\left\{e_{i}, f_{i}, h_{i} \mid i \in J_{\ell}\right\}$ is a finite-dimensional simple Lie algebra of the same type as $R[\mathrm{H}$, Theorem 18.3]. So we identify this subalgebra with the Lie algebra $\mathcal{G}$ as in Subsection 2.1 with Cartan subalgebra $\mathcal{H}=\bigoplus_{i \in J_{\ell}} \mathbb{C} h_{i}$, and the corresponding root system $R$. This then also allows us to identify the $3 \ell$ generators $e_{i}, f_{i}, h_{i}, i \in J_{\ell}$, here with the corresponding elements in Subsection 2.1. Next let $\ell+1 \leq i \leq n$ and use Lemma 2.2 (i) to fix $j_{1}, \ldots, j_{n_{i}} \in J_{n}$ such that

$$
e_{i}:=\left[f_{j_{1}}, \ldots, f_{j_{n_{i}}}, e_{n}\right]
$$

is a non-zero element of $\mathcal{G}_{\alpha_{i}}$. For $a \in J_{\nu}$ define

$$
e_{i, a}^{ \pm}:=\frac{1}{2}\left[f_{j_{1}}, \ldots, f_{j_{n_{i}}}, h_{n, a}^{ \pm}, e_{n}\right] .
$$

Next we set

$$
\begin{aligned}
H & :=\operatorname{span}_{\mathbb{C}}\left\{h_{i, a}^{ \pm} \mid i \in J_{\ell}, a \in J_{\nu}\right\}, \\
Z_{h} & :=\operatorname{span}_{\mathbb{C}}\left\{\left[h_{i, a}^{ \pm}, h_{j, b}^{ \pm}\right] \mid i, j \in J_{\ell}, a, b \in J_{\nu}\right\}, \\
S_{g} & :=\operatorname{span}_{\mathbb{C}}\left\{e_{i}, f_{i}, h_{i}, h_{i, a}^{ \pm}, v^{r} \mid i \in J_{\ell}, a \in J_{\nu}, r \in J_{m}\right\} .
\end{aligned}
$$

We also note that using (R2), one concludes that, for $r \in J_{m}$, the $\mathcal{G}$-submodule $\mathcal{V}^{r}$ of $\tilde{\mathcal{L}}$ generated by $v^{r}$ is an irreducible $\mathcal{G}$-module whose highest weight is the highest short root $\alpha_{n_{s}}$ of $R$ (see [H, Theorem 21.4]). Considering [2.11), we set

$$
v_{t}^{r}:=\left[f_{j_{1}^{t}}, \ldots, f_{j_{n_{t}}^{t}}, v^{r}\right] \text { and } v_{-t}^{r}=\left[f_{k_{1}^{t}}, \ldots, f_{k_{n_{t}^{t}}^{t}}, v^{r}\right] .
$$

Now we have the weight space decomposition

$$
\mathcal{V}^{r}=\bigoplus_{t=n_{\ell}+1}^{n_{s}}\left(\mathbb{C} v_{ \pm t}^{r}\right) \oplus \mathcal{V}_{0}^{r},
$$

where $\mathcal{V}_{0}^{r}$ is the corresponding zero weight space.

For $h=\sum_{i=1}^{\ell} r_{i} h_{i} \in \mathcal{H}$ and $a \in J_{\nu}$, take

$$
h_{a}^{ \pm}:=\sum_{i=1}^{\ell} r_{i} h_{i, a}^{ \pm},
$$

and for $\ell+1 \leq t \leq n$, set

$$
h_{t, a}^{ \pm}:=\left(h_{t}\right)_{a}^{ \pm} .
$$


Let $\sigma=\left(m_{1}, \ldots, m_{\nu}\right) \in \mathbb{Z}^{\nu}$. We call $|\sigma|:=\sum_{t=1}^{\nu}\left|m_{t}\right|$ the norm of $\sigma$. Let $\sigma \neq 0$ and $1 \leq i_{1}<\cdots<i_{p} \leq \nu$ be all $i_{j} \in J_{\nu}$ for which $m_{i_{j}} \neq 0$. Then $|\sigma|=\sum_{j=1}^{p}\left|m_{i_{j}}\right|$. For $1 \leq t \leq n$, we set

$$
\mathfrak{b}_{\sigma}^{t}:=(\underbrace{h_{t, i_{1}}^{\operatorname{sgn}\left(m_{i_{1}}\right)}, \ldots, h_{t, i_{1}}^{\operatorname{sgn}\left(m_{i_{1}}\right)}}_{\left|m_{i_{1}}\right|}, \ldots, \underbrace{h_{t, i_{p}}^{\operatorname{sgn}\left(m_{i_{p}}\right)}, \ldots, h_{t, i_{p}}^{\operatorname{sgn}\left(m_{i_{p}}\right)}}_{\left|m_{i_{p}}\right|})
$$

where $\operatorname{sgn}(m)$ for $m \in \mathbb{Z}$ is the sign of $m$. In fact $\mathfrak{b}_{\sigma}^{t}=\left(b_{1}^{t}, \ldots, b_{|\sigma|}^{t}\right)$ where for $k \in J_{|\sigma|}$,

$$
b_{k}^{t}:= \begin{cases}h_{t, i_{a}}^{+} & \text {if } m_{i_{a}}>0, \\ h_{t, i_{a}}^{-} & \text {if } m_{i_{a}}<0,\end{cases}
$$

in which $a$ is the unique element of $\{1, \ldots, p\}$ with

$$
1+\sum_{j=1}^{a-1}\left|m_{i_{j}}\right| \leq i \leq \sum_{j=1}^{a}\left|m_{i_{j}}\right|
$$

We also set $\mathfrak{b}_{0}^{t}:=\left(h_{t, 1}^{-}, h_{t, 1}^{+}\right)$. We call $\mathfrak{b}_{\sigma}:=\mathfrak{b}_{\sigma}^{n}$ the norm-tuple of $\sigma$.

Convention 3.1. For $\sigma \in \mathbb{Z}^{\nu}$, we denote the norm-tuple of $\sigma$ by $\mathfrak{b}_{\sigma}=\left(\mathfrak{b}_{1}^{\sigma}, \ldots, \mathfrak{b}_{|\sigma|}^{\sigma}\right)$ and by $\left(\mathfrak{b}_{1}, \ldots, \mathfrak{b}_{|\sigma|}\right)$ if there is no confusion. For $h \in \mathcal{H}$, if $i \in J_{|\sigma|}$, define $h^{i, \sigma}$ to be $h_{a}^{ \pm}$(see (3.6) ) if $\mathfrak{b}_{i}^{\sigma}=h_{n, a}^{ \pm}$for some $a \in J_{\nu}$. Also with an abuse of notation we write

$$
\left[r \mathfrak{b}_{\sigma}^{t}, x\right]:=\left[r \mathfrak{b}_{1}^{t}, \ldots, r \mathfrak{b}_{|\sigma|}^{t}, x\right] \quad\left(t \in J_{n}, x \in \tilde{\mathcal{L}}, r \in \mathbb{C}\right)
$$

We now introduce some more relations:

$$
\left[e_{i, a}^{ \pm}, f_{i}\right]=h_{i, a}^{ \pm} ; \quad i \in J_{\ell}, a \in J_{\nu}
$$

(R4) $\quad[H, \mathcal{H}]=\{0\}, \quad\left[S_{g}, Z_{h}\right]=\{0\}$.

(R5) $\quad\left[\left\langle\alpha_{t}, \alpha_{j}\right\rangle h_{i, a}^{ \pm}-\left\langle\alpha_{t}, \alpha_{i}\right\rangle h_{j, a}^{ \pm}, \mathbb{C} e_{t}+\mathbb{C} f_{t}\right]=0 ; \quad a \in J_{\nu}, i, j \in J_{\ell}, t \in J_{n}$.

\section{Canceling relations:}

$$
\begin{aligned}
& {\left[h_{i, a}^{-}, h_{j, a}^{+}, e_{t}\right]=\left\langle\alpha_{t}, \alpha_{i}\right\rangle\left\langle\alpha_{t}, \alpha_{j}\right\rangle e_{t}, \quad\left[h_{i, a}^{-}, h_{j, a}^{+}, f_{t}\right]=\left\langle\alpha_{t}, \alpha_{i}\right\rangle\left\langle\alpha_{t}, \alpha_{j}\right\rangle f_{t} ;} \\
& a \in J_{\nu}, i, j, t \in J_{n} .
\end{aligned}
$$

We consider 3.5 to define our next two sets of relations:

(R7)

$$
\begin{aligned}
& {\left[\left\langle\alpha_{t}, \alpha_{j}\right\rangle h_{i, a}^{ \pm}-\left\langle\alpha_{t}, \alpha_{i}\right\rangle h_{j, a}^{ \pm}, \mathbb{C} v_{ \pm t}^{r}\right]=0 ;} \\
& a \in J_{\nu}, r \in J_{m}, i, j \in J_{\ell}, n_{\ell}+1 \leq t \leq n_{s} .
\end{aligned}
$$




\section{Canceling module relations:}

(R8)

$$
\begin{aligned}
& {\left[h_{i, a}^{-}, h_{j, a}^{+}, v_{ \pm t}^{r}\right]=\left\langle\alpha_{t}, \alpha_{i}\right\rangle\left\langle\alpha_{t}, \alpha_{j}\right\rangle v_{ \pm t}^{r} ;} \\
& a \in J_{\nu}, i, j \in J_{\ell}, r \in J_{m}, n_{\ell}+1 \leq t \leq n_{s} .
\end{aligned}
$$

Finally we recall (2.19) and (3.7) to state our last set of relations:

\section{Basic short part relations:}

$$
\begin{aligned}
& {\left[v^{r}, v_{ \pm i}^{s}\right]=m_{ \pm i}^{\prime} a_{r, s}^{\prime}\left[\frac{1}{2} \mathfrak{b}_{\sigma_{r, s}^{\prime}}^{\prime}, e_{t_{ \pm i}^{\prime}}^{\prime}\right]+m_{ \pm i} a_{r, s}\left[\frac{1}{2} \mathfrak{b}_{\sigma_{r, s}}^{t_{i}}, v_{t_{i}}^{t_{r, s}}\right]} \\
& r, s \in J_{m}, n_{\ell}+1 \leq i \leq n_{s}-1 .
\end{aligned}
$$

Remark. It is known that $\left\langle\alpha_{i}, \alpha_{j}\right\rangle=\alpha_{i}\left(h_{j}\right)$ for $i, j \in J_{n}$, so we may use relations (R1)-(R9) with $\alpha_{i}\left(h_{j}\right)$ instead of $\left\langle\alpha_{i}, \alpha_{j}\right\rangle$.

Definition 3.1. Starting from a centerless Lie torus $\mathcal{L}$ with the structural data $\mathfrak{D}$, we call the Lie algebra $\tilde{\mathcal{L}}$ defined by generators 3.1 and relations (R1)-(R9) the presented Lie algebra associated to $\mathcal{L}$ (or $\mathfrak{D}$ ).

We are now ready to state our main theorem.

Main Theorem. Let $\mathcal{L}$ be a centerless Lie torus of type $X \neq A, C, B C$ with the universal covering $\mathfrak{A}$, and associated presented Lie algebra $\tilde{\mathcal{L}}$. Then $\tilde{\mathcal{L}} \cong \mathfrak{A}$. In particular $\mathfrak{A}$ is a finitely presented Lie algebra.

In the remaining part of this section we establish several results which are needed prior to the proof of the main theorem. The proof proper will be presented in Section 4. An outline of the proof is as follows. We first show that $\tilde{\mathcal{L}}$ as a $\mathcal{G}$ module $(\mathcal{G}$ is a subalgebra of $\tilde{\mathcal{L}})$ is a direct sum of irreducible $\mathcal{G}$-modules whose highest weights belong to $R$. We then use this to show that $\tilde{\mathcal{L}}$ is an $R$-graded Lie algebra. Using the fact that both $\tilde{\mathcal{L}}$ and $\mathfrak{A}$ are $R$-graded Lie algebras and invoking the structure of $R$-graded Lie algebras, we prove that $\tilde{\mathcal{L}}$ is a central extension of $\mathfrak{A}$, and finally we prove that $\mathfrak{A}$ is isomorphic to $\tilde{\mathcal{L}}$.

We recall that $\mathfrak{A}$ is generated by 2.18 and so one can easily deduce from Subsection 2.2 that there exists a Lie algebra epimorphism $\psi: \tilde{\mathcal{L}} \rightarrow \mathfrak{A}$ as follows:

$$
\begin{aligned}
& e_{i} \mapsto e_{i} \otimes 1, \quad f_{i} \mapsto f_{i} \otimes 1, \quad h_{i} \mapsto h_{i} \otimes 1, \quad a \in J_{\nu}, i \in J_{\ell}, r \in J_{m} \\
& h_{i, a}^{ \pm} \mapsto h_{i} \otimes t_{a}^{ \pm 1}, \quad v^{r} \mapsto v_{n_{s}} \otimes w_{r} ;
\end{aligned}
$$

We shall prove $\psi$ is an isomorphism. From now on we fix $\sigma=\left(m_{1}, \ldots, m_{\nu}\right) \in \mathbb{Z}^{\nu}$ and associate to $\sigma$ the following elements of $\tilde{\mathcal{L}}$ :

$$
e_{\sigma}:=\left[\frac{1}{2} \mathfrak{b}_{\sigma}, e_{n}\right], \quad v_{\sigma}^{r}:=\left[\mathfrak{b}_{\sigma}, v^{r}\right] ; \quad r \in J_{m} \text { (see Convention 3.1). }
$$


Using (R6) and (R8), we have

$$
e_{0}=e_{n} \quad \text { and } \quad v_{0}^{r}=v^{r} .
$$

Now considering $\tilde{\mathcal{L}}$ as a $\mathcal{G}$-module, we set

$$
\begin{aligned}
& \mathcal{G}_{\sigma}:=\text { the } \mathcal{G} \text {-submodule of } \tilde{\mathcal{L}} \text { generated by } e_{\sigma}, \\
& \mathcal{V}_{\sigma}^{r}:=\text { the } \mathcal{G} \text {-submodule of } \tilde{\mathcal{L}} \text { generated by } v_{\sigma}^{r} .
\end{aligned}
$$

Proposition 3.1. For $\alpha \in R^{\times}$, set

$$
\tilde{\mathcal{L}}_{\alpha}^{0}:= \begin{cases}\mathcal{G}_{\alpha}+\sum_{r=1}^{m} \mathcal{V}_{\alpha}^{r} & \text { if } \alpha \in R_{\mathrm{sh}} \\ \mathcal{G}_{\alpha} & \text { if } \alpha \in R_{\mathrm{lg}}\end{cases}
$$

(i) Recall (3.6) and let $a \in J_{\nu}, x, y \in \mathcal{H}$ and $t \in J_{n}$. Then

$$
\left[\alpha_{t}(x) y_{a}^{ \pm}-\alpha_{t}(y) x_{a}^{ \pm}, \tilde{\mathcal{L}}_{ \pm \alpha_{t}}^{0}\right]=\{0\} ;
$$

in particular if $x \in \mathcal{H}$ is such that $\alpha_{t}(x)=0$, then $\left[x_{a}^{ \pm}, \tilde{\mathcal{L}}_{ \pm \alpha_{t}}^{0}\right]=\{0\}$.

(ii) Let $p \in \mathbb{Z}^{>0}, a_{1}, \ldots, a_{p} \in J_{\nu}$, and $t \in J_{n}$, and suppose that $x_{j}, y_{j} \in \mathcal{H}$ are such that $\alpha_{t}\left(y_{j}\right) \neq 0$ for $j \in J_{p}$. Then for $e \in \tilde{\mathcal{L}}_{ \pm \alpha_{t}}^{0}$, we have

$$
\left[\left(x_{1}\right)_{a_{1}}^{ \pm}, \ldots,\left(x_{p}\right)_{a_{p}}^{ \pm}, e\right]=\prod_{j=1}^{p}\left(\alpha_{t}\left(x_{j}\right) / \alpha_{t}\left(y_{j}\right)\right)\left[\left(y_{1}\right)_{a_{1}}^{ \pm}, \ldots,\left(y_{p}\right)_{a_{p}}^{ \pm}, e\right]
$$

with the same sign in $\left(x_{j}\right)_{a_{j}}^{ \pm}$and $\left(y_{j}\right)_{a_{j}}^{ \pm}$for $j \in J_{p}$.

(iii) Let $p, q \in \mathbb{Z}^{>0}, a_{1}, \ldots, a_{p}, b_{1}, \ldots, b_{q} \in J_{\nu}$, and $t, t^{\prime} \in J_{n}$ with $t \neq t^{\prime}$, and suppose that $x_{j}, y_{j} \in \mathcal{H}, j \in J_{p}$, are such that $\alpha_{t}\left(y_{j}\right) \neq 0$ and $\alpha_{t^{\prime}}\left(y_{j}\right)=0$. Then for $z_{j} \in \mathcal{H}$ with $\alpha_{t}\left(z_{j}\right)=0, j \in J_{q}, e \in \tilde{\mathcal{L}}_{ \pm \alpha_{t}}^{0}$ and $f \in \tilde{\mathcal{L}}_{ \pm \alpha_{t^{\prime}}}^{0}$, we have

$$
\begin{aligned}
& {\left[\left[\left(z_{1}\right)_{b_{1}}^{ \pm}, \ldots,\left(z_{q}\right)_{b_{q}}^{ \pm}, f\right],\left[\left(x_{1}\right)_{a_{1}}^{ \pm}, \ldots,\left(x_{p}\right)_{a_{p}}^{ \pm}, e\right]\right]} \\
& \quad=\prod_{j=1}^{p}\left(\alpha_{t}\left(x_{j}\right) / \alpha_{t}\left(y_{j}\right)\right)\left[\left(z_{1}\right)_{b_{1}}^{ \pm}, \ldots,\left(z_{q}\right)_{b_{q}}^{ \pm},\left(y_{1}\right)_{a_{1}}^{ \pm}, \ldots,\left(y_{p}\right)_{a_{p}}^{ \pm},[f, e]\right]
\end{aligned}
$$

with the same sign in $\left(x_{j}\right)_{a_{j}}^{ \pm}$and $\left(y_{j}\right)_{a_{j}}^{ \pm}$for $j \in J_{p}$.

Proof. (i) The first expression is immediate using (R5) and (R7). For the second expression, we note that as $\alpha_{t} \neq 0$, one finds $y \in \mathcal{H}$ such that $\alpha_{t}(y) \neq 0$. Now the statement holds by considering the first expression.

(ii) Using (i), we have for all $j \in J_{p}$,

$$
\left[\left(x_{j}\right)_{a_{j}}^{ \pm}, e\right]=\left(\alpha_{t}\left(x_{j}\right) / \alpha_{t}\left(y_{j}\right)\right)\left[\left(y_{j}\right)_{a_{j}}^{ \pm}, e\right] .
$$

Now we are done using the Jacobi identity together with (R4).

(iii) Using (i), we deduce that for all $i \in J_{p}$ and $j \in J_{q},\left[\left(y_{i}\right)_{a_{i}}^{ \pm}, f\right]=0$ and $\left[\left(z_{j}\right)_{b_{j}}^{ \pm}, e\right]=0$. Now (R4) together with the Jacobi identity implies that 
$\left[\left[\left(z_{1}\right)_{b_{1}}^{ \pm}, \ldots,\left(z_{q}\right)_{b_{q}}^{ \pm}, f\right],\left(y_{i}\right)_{a_{i}}^{ \pm}\right]=0$ for all $i \in J_{p}$. Also using (ii), we can replace $\left[\left(x_{1}\right)_{a_{1}}^{ \pm}, \ldots,\left(x_{p}\right)_{a_{p}}^{ \pm}, e\right]$ with an expression as on the right hand side of the display appearing in (ii). Now we are done using these together with the Jacobi identity.

The following proposition determines, up to isomorphism, the modules $\mathcal{G}_{\sigma}$ and $\mathcal{V}_{\sigma}^{r}$ appearing in 3.10 .

Proposition 3.2. (i) $\mathcal{G}_{\sigma}$ is an irreducible finite-dimensional $\mathcal{G}$-module with highest pair $\left(e_{\sigma}, \alpha_{n}\right)$. In fact, $\mathcal{G}_{\sigma} \cong \mathcal{G}$ as $\mathcal{G}$-modules.

(ii) For $r \in J_{m}, \mathcal{V}_{\sigma}^{r}$ is an irreducible finite-dimensional $\mathcal{G}$-module with highest pair $\left(v_{\sigma}^{r}, \alpha_{n_{s}}\right)$. In fact, $\mathcal{V}_{\sigma}^{r} \cong \mathcal{V}$ as $\mathcal{G}$-modules.

Proof. (i) From (3.8), one can see that $\psi\left(e_{\sigma}\right)=e_{n} \otimes t^{\sigma} \neq 0$. Therefore $e_{\sigma} \neq 0$. Thus by [H, Theorem 21.4], it is enough to show that, for all $i \in J_{\ell}$,

$$
\left[h_{i}, e_{\sigma}\right]=\alpha_{n}\left(h_{i}\right) e_{\sigma}, \quad\left[e_{i}, e_{\sigma}\right]=0, \quad\left[f_{i}^{\alpha_{n}\left(h_{i}\right)+1}, e_{\sigma}\right]=0 .
$$

Fix $i \in J_{\ell}$. We first note that the equalities in (3.11) hold for $\sigma=0$ as $e_{n}$ is a maximal vector in the $\mathcal{G}$-module $\mathcal{G}$. Now let $\sigma$ be non-zero with norm-tuple $\mathfrak{b}_{\sigma}=\left(\mathfrak{b}_{1}, \ldots, \mathfrak{b}_{|\sigma|}\right)$. Considering Convention 3.1. one deduces from (R4) together with (an iterated use of) the Jacobi identity that

$$
\left[h_{i}, e_{\sigma}\right]=\left[h_{i},\left[\frac{1}{2} \mathfrak{b}_{\sigma}, e_{n}\right]\right]=-\left[\frac{1}{2} \mathfrak{b}_{\sigma},\left[e_{n}, h_{i}\right]\right]=\alpha_{n}\left(h_{i}\right) e_{\sigma} .
$$

To prove the next two equalities in (3.11), we use induction on $|\sigma|$. Assume $0 \neq \sigma$ is non-zero with $\left[e_{i}, e_{\sigma}\right]=0=\left[f_{i}^{\alpha_{n}\left(h_{i}\right)+1}, e_{\sigma}\right]$. We are done if we show that for $a_{0} \in J_{\nu}$,

$$
\left[e_{i},\left[\frac{1}{2} h_{n, a_{0}}^{ \pm}, e_{\sigma}\right]\right]=0=\left[f_{i}^{\alpha_{n}\left(h_{i}\right)+1},\left[\frac{1}{2} h_{n, a_{0}}^{ \pm}, e_{\sigma}\right]\right] .
$$

Since $\alpha_{n}$ and $\alpha_{i}$ are not proportional, there exists $x \in \mathcal{H}$ such that $\alpha_{n}(x) \neq 0$ and $\alpha_{i}(x)=0$. Therefore using (R4) and Proposition 3.1(i), we have

$$
\left[\frac{1}{2} h_{n, a_{0}}^{ \pm}, e_{\sigma}\right] \in \mathbb{C}\left[x_{a_{0}}^{ \pm}, e_{\sigma}\right] \quad \text { and } \quad\left[x_{a_{0}}^{ \pm}, e_{i}\right]=0 .
$$

Now using these together with the Jacobi identity and the induction hypothesis, we get

$$
\left[e_{i},\left[\frac{1}{2} h_{n, a_{0}}^{ \pm}, e_{\sigma}\right]\right] \in \mathbb{C}\left[e_{i},\left[x_{a_{0}}^{ \pm}, e_{\sigma}\right]\right]=\mathbb{C}\left[\left[x_{a_{0}}^{ \pm}, e_{i}\right], e_{\sigma}\right]=0=\left[\left[x_{a_{0}}^{ \pm}, f_{i}\right], e_{\sigma}\right]
$$

and

$$
\left[f_{i}^{\alpha_{n}\left(h_{i}\right)+1}, \frac{1}{2} h_{n, a_{0}}^{ \pm}, e_{\sigma}\right] \in \mathbb{C}\left[f_{i}^{\alpha_{n}\left(h_{i}\right)+1}, x_{a_{0}}^{ \pm}, e_{\sigma}\right]=\mathbb{C}\left[x_{a_{0}}^{ \pm}, f_{i}^{\alpha_{n}\left(h_{i}\right)+1}, e_{\sigma}\right]=0 .
$$

(ii) Use an argument analogous to the one in (i). 
Using Proposition 3.2(i), one concludes that there exists a $\mathcal{G}$-module isomorphism $\varphi_{\sigma}: \mathcal{G} \rightarrow \mathcal{G}_{\sigma}$ mapping $e_{n}$ to $e_{\sigma}$. Therefore $\mathcal{G}_{\sigma}$ admits a weight space decomposition $\mathcal{G}_{\sigma}=\sum_{\gamma \in R}\left(\mathcal{G}_{\sigma}\right)_{\gamma}$, where $\left(\mathcal{G}_{\sigma}\right)_{\gamma}=\varphi_{\sigma}\left(\mathcal{G}_{\gamma}\right), \gamma \in R$. For $a \in J_{\nu}$, we set

$$
\sigma_{a}^{ \pm}:=(0, \ldots, 0, \underbrace{ \pm 1}_{a \text {-th }}, 0, \ldots, 0) \text { and } \varphi_{a}^{ \pm}:=\varphi_{\sigma_{a}^{ \pm}} .
$$

From 3.9 we have $\varphi_{a}^{ \pm}\left(e_{n}\right)=e_{\sigma_{a}^{ \pm}}=\frac{1}{2}\left[h_{n, a}^{ \pm}, e_{n}\right]$. Now considering 3.2, 3.3 and (R3) we have, for $i \in J_{\ell}$ and $a \in J_{\nu}$,

$$
e_{i, a}^{ \pm}=\varphi_{a}^{ \pm}\left(e_{i}\right) \quad \text { and } \quad h_{i, a}^{ \pm}=\varphi_{a}^{ \pm}\left(h_{i}\right)
$$

Next set, for $t \in J_{n}, a \in J_{\nu}$ and $\ell+1 \leq j \leq n$,

$$
\begin{aligned}
e_{t, \sigma}:=\varphi_{\sigma}\left(e_{t}\right), & f_{t, \sigma}:=\varphi_{\sigma}\left(f_{t}\right), & h_{t, \sigma}:=\varphi_{\sigma}\left(h_{t}\right), \\
f_{t, a}^{ \pm}:=\varphi_{a}^{ \pm}\left(f_{t}\right), & e_{j, a}^{ \pm}:=\varphi_{a}^{ \pm}\left(e_{j}\right), & h_{j, a}:=\varphi_{a}^{ \pm}\left(h_{j}\right)
\end{aligned}
$$

and finally set

$$
\mathcal{Z}:=\operatorname{span}_{\mathbb{C}}\left\{\left[h_{i, \sigma}, h_{i, \tau}\right] \mid i \in J_{\ell}, \sigma, \tau \in \mathbb{Z}^{\nu}\right\}
$$

We next note that as $\varphi_{\sigma}, \sigma \in \mathbb{Z}^{\nu}$, is a $\mathcal{G}$-module isomorphism and $\left[e_{i}, f_{j}\right]=\delta_{i, j} h_{i}$, $i, j \in J_{\ell}$, we get, for $a \in J_{\nu}$ and $i, j \in J_{\ell}$,

$$
\left[e_{i, \sigma}, f_{j}\right]=-\left[f_{i, \sigma}, e_{j}\right]=\delta_{i, j} h_{i, \sigma} .
$$

Now let $r \in J_{m}$. Then Proposition $3.2($ ii) guarantees the existence of a $\mathcal{G}$ module isomorphism

$$
\psi_{\sigma}^{r}: \mathcal{V} \rightarrow \mathcal{V}_{\sigma}^{r} \quad \text { such that } \quad \mathbf{v}=v_{n_{s}} \mapsto v_{\sigma}^{r}(\text { see }(3.9))
$$

Set $\psi_{r}:=\psi_{0}^{r}$. Since $\psi_{r}(\mathbf{v})=v^{r}, 2.11$ and 3.5 imply that

$$
v_{ \pm t}^{r}=\psi_{r}\left(v_{ \pm t}\right) ; \quad n_{\ell}+1 \leq t \leq n_{s} .
$$

Also as $\mathcal{V}_{\sigma}^{r}$ is an irreducible finite-dimensional $\mathcal{G}$-module of highest weight $\alpha_{n_{s}}$, we have

$$
\begin{aligned}
& \mathcal{V}_{\sigma}^{r}=\left(\mathcal{V}_{\sigma}^{r}\right)_{0} \oplus \bigoplus_{t=n_{\ell}+1}^{n_{s}}\left(\mathcal{V}_{\sigma}^{r}\right)_{ \pm t} \quad \text { where } \\
& \left(\mathcal{V}_{\sigma}^{r}\right)_{ \pm t}:=\left(\mathcal{V}_{\sigma}^{r}\right)_{ \pm \alpha_{t}}=\mathbb{C} v_{\sigma, \pm t}^{r}, \quad v_{\sigma, \pm t}^{r}:=\psi_{\sigma}^{r}\left(v_{ \pm t}\right) ; \quad n_{\ell}+1 \leq t \leq n_{s} .
\end{aligned}
$$

Proposition 3.3. Let $t \in J_{n}$ and $\alpha= \pm \alpha_{t}$. Suppose for $j \in J_{|\sigma|}, x_{j} \in \mathcal{H}$ is such that $\alpha\left(x_{j}\right) \neq 0$. For $j \in J_{|\sigma|}$, recall Convention 3.1 and set $c_{j}:=\left(x_{j}\right)^{j, \sigma}$. Then for 
$e \in \tilde{\mathcal{L}}_{\alpha}^{0}$ (see Proposition 3.1 , we have

$$
\frac{1}{\prod_{j=1}^{|\sigma|}\left( \pm \alpha\left(x_{j}\right)\right)}\left[c_{1}, \ldots, c_{|\sigma|}, e\right]= \begin{cases}\varphi_{\sigma}(e) & \text { if } e \in \mathcal{G}_{\alpha} \\ \psi_{\sigma}^{r}(e) & \text { if } e \in \mathcal{V}_{\alpha}^{r} \text { for some } r \in J_{m}\end{cases}
$$

Proof. Using Proposition 3.1(ii), it is enough to show that the right hand side of the expression equals $\left[y_{1}^{1, \sigma}, \ldots, y_{|\sigma|}^{|\sigma|, \sigma}, e\right]$ for some $y_{1}, \ldots, y_{|\sigma|} \in \mathcal{H}$ with $\alpha\left(y_{i}\right)=1$, $i \in J_{|\sigma|}$. Take

$$
\begin{cases}v:=e_{n} \text { and } \lambda:=\alpha_{n} & \text { if } e \in \mathcal{G}_{\alpha} \\ v:=v^{r} \text { and } \lambda:=\alpha_{n_{s}} & \text { if } e \in \mathcal{V}_{\alpha}^{r} \text { for some } r \in J_{m} .\end{cases}
$$

Using Lemma $2.2(\mathrm{i})$, we may suppose $e=\left[f_{i_{p}}, \ldots, f_{i_{1}}, v\right]$ where $p \in \mathbb{N}, i_{1}, \ldots, i_{p}$ $\in J_{n}$ and for $q \in J_{p}, \alpha_{i_{q}} \neq \pm\left(\lambda-\sum_{r=1}^{q-1} \alpha_{i_{r}}\right)$.

For $1 \leq q \leq p, \alpha_{i_{q}} \neq \pm\left(\lambda-\sum_{r=1}^{q-1} \alpha_{i_{r}}\right)$, so there is $x_{q} \in \mathcal{H}$ such that $\alpha_{i_{q}}\left(x_{q}\right)=0$ and $\left(\lambda-\sum_{r=1}^{q-1} \alpha_{i_{r}}\right)\left(x_{q}\right)=1$. Now we recall Convention 3.1 and note that Proposition 3.1(i) implies that $\left[f_{i_{q}}, x_{q}^{i, \sigma}\right]=0, i \in J_{|\sigma|}$. So invoking Proposition 3.1(ii) together with the Jacobi identity, we get

$$
\begin{aligned}
& {\left[f_{i_{q}}, x_{q}^{1, \sigma}, \ldots, x_{q}^{|\sigma|, \sigma}, f_{i_{q-1}}, \ldots, f_{i_{1}}, v\right] }=\left[x_{q}^{1, \sigma}, \ldots, x_{q}^{|\sigma|, \sigma}, f_{i_{q}}, f_{i_{q-1}}, \ldots, f_{i_{1}}, v\right] \\
& \stackrel{\text { if } q \neq p}{=}\left[x_{q+1}^{1, \sigma}, \ldots, x_{q+1}^{|\sigma|, \sigma}, f_{i_{q}}, f_{i_{q-1}}, \ldots, f_{i_{1}}, v\right]
\end{aligned}
$$

Using this repeatedly, one has

$$
\begin{aligned}
{\left[x_{p}^{1, \sigma}, \ldots, x_{p}^{|\sigma|, \sigma}, e\right] } & =\left[x_{p}^{1, \sigma}, \ldots, x_{p}^{|\sigma|, \sigma}, f_{i_{p}}, \ldots, f_{i_{1}}, v\right] \\
& =\left[f_{i_{p}}, \ldots, f_{i_{1}}, x_{1}^{1, \sigma}, \ldots, x_{1}^{|\sigma|, \sigma}, v\right] \\
& = \begin{cases}{\left[f_{i_{p}}, \ldots, f_{i_{1}}, e_{\sigma}\right]} & \text { if } e \in \mathcal{G}_{\alpha} \\
{\left[f_{i_{p}}, \ldots, f_{i_{1}}, v_{\sigma}^{r}\right]} & \text { if } e \in \mathcal{V}_{\alpha}^{r} \text { for some } r \in J_{m}\end{cases}
\end{aligned}
$$

Now we are done as $\varphi_{\sigma}$ and $\psi_{\sigma}^{r}$ are $\mathcal{G}$-module homomorphisms.

Recall from Section 2 that we have fixed a specific order for (simple) roots, in terms of their lengths. Accordingly, for type $F_{4}$, we consider the following fundamental system:

$$
\left\{\alpha_{1}=\varepsilon_{2}-\varepsilon_{3}, \alpha_{2}=\varepsilon_{3}-\varepsilon_{4}, \alpha_{3}=\varepsilon_{4}, \alpha_{4}=\frac{1}{2}\left(\varepsilon_{1}-\varepsilon_{2}-\varepsilon_{3}-\varepsilon_{4}\right)\right\},
$$

where as usual $\varepsilon_{i}$ 's are the standard orthogonal basis for $\mathbb{R}^{4}$. Using the module theory for type $F_{4}$, we may find complex numbers $a, b, a^{\prime}, b^{\prime}, a_{3}^{\prime \prime}, b_{3}^{\prime \prime}, a_{4}^{\prime \prime}, b_{4}^{\prime \prime}$ satisfying 


$$
\begin{aligned}
& \left(a_{3}^{\prime \prime} a^{\prime}+b_{3}^{\prime \prime} a\right) b=-\left(a_{3}^{\prime \prime} b^{\prime}+b_{3}^{\prime \prime} b\right) a, \quad\left(a_{4}^{\prime \prime} a^{\prime}+b_{4}^{\prime \prime} a\right) b^{\prime}=-\left(a_{4}^{\prime \prime} b^{\prime}+b_{4}^{\prime \prime} b\right) a^{\prime}, \\
& t\left(a^{\prime} f_{3} \cdot v_{3}+a f_{4} \cdot v_{4}, b^{\prime} f_{3} \cdot v_{3}+b f_{4} \cdot v_{4}\right)=1, \\
& \left(a^{\prime} f_{3} \cdot v_{3}+a f_{4} \cdot v_{4}\right) *\left(b^{\prime} f_{3} \cdot v_{3}+b, f_{4} \cdot v_{4}\right)=0, \\
& e_{i} \cdot\left(f_{3} \cdot v_{3}\right)=a_{i}^{\prime \prime} v_{i}, \quad e_{i} \cdot\left(f_{4} \cdot v_{4}\right)=b_{i}^{\prime \prime} v_{i},
\end{aligned}
$$

where $3 \leq i \leq 4$ and $t$ is the trace form introduced in Section 2 . We then define

$$
D_{\sigma, \tau}^{r, s}:= \begin{cases}{\left[a^{\prime}\left[f_{3}, v_{\sigma, 3}^{r}\right]+a\left[f_{4}, v_{\sigma, 4}^{r}\right], b^{\prime}\left[f_{3}, v_{\tau, 3}^{s}\right]+b\left[f_{4}, v_{\tau, 4}^{s}\right]\right]} & \text { for type } F_{4}, \\ {\left[\left[f_{\ell}, v_{\sigma, \ell}^{r}\right],\left[f_{\ell}, v_{\tau, \ell}^{s}\right]\right]} & \text { otherwise, }\end{cases}
$$

where $r, s \in J_{m}$ and $\sigma, \tau \in \mathbb{Z}^{\nu}$. Now set

$$
\mathcal{D}:=\operatorname{span}_{\mathbb{C}}\left\{D_{\sigma, \tau}^{r, s} \mid r, s \in J_{m}, \sigma, \tau \in \mathbb{Z}^{\nu}\right\} .
$$

In Propositions 3.4 and 3.5 below, we prove several crucial relations among the elements of $\tilde{\mathcal{L}}$, which reveal the algebra structure between parts $\mathcal{G}_{\sigma}, \mathcal{V}_{\sigma}^{r}, \mathcal{D}$ and $\mathcal{Z}$ (see $3.10,3.15$ ) and 3.22 ) of $\tilde{\mathcal{L}}$. Since the proofs of these two propositions are quite technical and a bit long, we postpone them until Section 5 .

Proposition 3.4. Let $r \in J_{m}, a \in J_{\nu}, i \neq j \in J_{\ell}$ and $\sigma, \tau \in \mathbb{Z}^{\nu}$. Set $\tau^{ \pm a}=\tau \pm \sigma_{a}$. Then considering (3.14, we have

(i) $\left[e_{i, \tau}, e_{i, \sigma}\right]=0=\left[f_{i, \tau}, f_{i, \sigma}\right]$,

(ii) $\left[e_{i, \sigma}, h_{j, \tau}\right]=-\alpha_{i}\left(h_{j}\right) e_{i, \sigma+\tau}$ and $\left[f_{i, \sigma}, h_{j, \tau}\right]=\alpha_{i}\left(h_{j}\right) f_{i, \sigma+\tau}$,

(iii) $\left[h_{i, \tau}, e_{i, a}^{ \pm}\right]=\left[h_{i, a}^{ \pm}, e_{i, \tau}\right]=2 e_{i, \tau^{ \pm a}}$ and $\left[h_{i, \tau}, f_{i, a}^{ \pm}\right]=\left[h_{i, a}^{ \pm}, f_{i, \tau}\right]=-2 f_{i, \tau^{ \pm a}}$,

(iv) $\left[h_{j, a}^{ \pm}, h_{i, \sigma}\right]=\left(\alpha_{i}\left(h_{j}\right) / 2\right)\left[h_{i, a}^{ \pm}, h_{i, \sigma}\right]$,

(v) $\left[e_{i, a}^{ \pm}, f_{i, \tau}\right]=\frac{1}{2}\left[h_{i, a}^{ \pm}, h_{i, \tau}\right]+h_{i, \tau \pm a}$,

(vi) $\left[v^{r}, h_{i, \sigma}\right]=-\alpha_{n_{s}}\left(h_{i}\right) v_{\sigma}^{r}$, in particular

$$
\left[h_{i, \sigma}, h_{i, \tau}, v^{r}\right]=\left[h_{i, \tau}, h_{i, \sigma}, v^{r}\right] .
$$

Proposition 3.5. For $\alpha \in \mathcal{H}^{\star} \backslash\{0\}, \sigma \in \mathbb{Z}^{\nu}$ and $r \in J_{m}$, define $\left(\mathcal{G}_{\sigma}\right)_{\alpha}$ and $\left(\mathcal{V}_{\sigma}^{r}\right)_{\alpha}$ to be zero if $\alpha$ is not a root or a short root respectively. Let $\sigma, \tau \in \mathbb{Z}^{\nu}, r, s \in J_{m}$ and $t \in J_{n}$. Then we have:
(i) $\left[H, \sum_{t^{\prime}=1}^{n} \sum_{\sigma \in \mathbb{Z}^{\nu}}\left(\mathcal{G}_{\sigma}\right)_{ \pm \alpha_{t^{\prime}}}\right] \subseteq \sum_{t^{\prime}=1}^{n} \sum_{\sigma \in \mathbb{Z}^{\nu}}\left(\mathcal{G}_{\sigma}\right)_{ \pm \alpha_{t^{\prime}}}$.
(ii) $\left[v^{r},\left(\mathcal{G}_{\sigma}\right)_{ \pm \alpha_{t}}\right] \subseteq\left(\mathcal{V}_{\sigma}^{r}\right)_{ \pm \alpha_{t}+\alpha_{n_{s}}}$ and $\left[v^{r},\left(\mathcal{G}_{\sigma}\right)_{0}\right] \subseteq\left(\mathcal{V}_{\sigma}^{r}\right)_{\alpha_{n_{s}}}$.
(iii) $\left[H,\left(\mathcal{V}_{\sigma}^{r}\right)_{0}\right]=\{0\}$.
(iv) $\left[H, \sum_{p=1}^{m} \sum_{\delta \in \mathbb{Z}^{\nu}} \mathcal{V}_{\delta}^{p}\right] \subseteq \sum_{p=1}^{m} \sum_{\delta \in \mathbb{Z}^{\nu}} \mathcal{V}_{\delta}^{p}$.
(v) $\left[v^{s}, v_{\sigma}^{r}\right]=0$. 
(vi) For $\alpha \in R_{\text {sh }} \cup\{0\} \backslash\left\{ \pm \alpha_{n_{s}}\right\}$, we have

$$
\left[v^{s},\left(\mathcal{V}_{\sigma}^{r}\right)_{\alpha}\right] \subseteq \sum_{\delta \in \mathbb{Z}^{\nu}}\left(\mathcal{G}_{\delta}\right)_{\alpha+\alpha_{n_{s}}}+\sum_{1 \leq p \leq m} \sum_{\delta \in \mathbb{Z}^{\nu}}\left(\mathcal{V}_{\delta}^{p}\right)_{\alpha+\alpha_{n_{s}}} .
$$

Moreover only the first [second] summation on the right hand side occurs if $r=s[r \neq s]$.

(vii) $\left[v^{s}, v_{\sigma,-n_{s}}^{r}\right] \subseteq \sum_{\delta \in \mathbb{Z}^{\nu}}\left(\mathcal{G}_{\delta}\right)_{0}+\sum_{1 \leq k \leq m} \sum_{\delta \in \mathbb{Z}^{\nu}}\left(\mathcal{V}_{\delta}^{k}\right)_{0}+\mathcal{D}$.

(viii) $\left[v^{r}, \mathcal{D}\right] \subseteq \sum_{k=1}^{m} \sum_{\delta \in \mathbb{Z}^{\nu}} \mathcal{V}_{\delta}^{k}$.

(ix) $[H, \mathcal{D}]=\{0\}$.

(x) $\mathcal{D}$ is a trivial $\mathcal{G}$-module.

(xi) Considering 3.15 , we have $\mathcal{Z} \subseteq Z(\tilde{\mathcal{L}})$.

\section{$\S 4$. Main theorem}

In this section we state and prove the main theorem of this work. The notation and terminology will be as in the previous sections.

Theorem 4.1 (Main Theorem). Let $\mathcal{L}$ be a centerless Lie torus of type $X \neq$ $A, C, B C$ with the universal covering $\mathfrak{A}$, and associated presented Lie algebra $\tilde{\mathcal{L}}$. Then $\psi: \tilde{\mathcal{L}} \rightarrow \mathfrak{A}$ (see 3.8$)$ ) is an isomorphism. In particular $\mathfrak{A}$ is a finitely presented Lie algebra.

Proof. We proceed in a few steps.

Step 1. Considering (3.13) and Propositions 3.2, 3.4(iv) together with Proposition 3.5 and using the same argument as in [You, Theorem 2.2 and Proposition 2.11], we find that

$$
\tilde{\mathcal{L}}=\sum_{\sigma \in \mathbb{Z}^{\nu}} \mathcal{G}_{\sigma}+\sum_{\sigma \in \mathbb{Z}^{\nu}} \sum_{r \in J_{m}} \mathcal{V}_{\sigma}^{r}+\mathcal{D}+\mathcal{Z} \quad \text { and } \quad Z(\tilde{\mathcal{L}}) \subseteq \mathcal{D}+\mathcal{Z}
$$

and that $\tilde{\mathcal{L}}$ is an $R$-graded Lie algebra. Moreover $\tilde{\mathcal{L}}=\bigoplus_{\alpha \in R} \tilde{\mathcal{L}}_{\alpha}$ where

$$
\tilde{\mathcal{L}}_{\alpha}= \begin{cases}\sum_{\sigma \in \mathbb{Z}^{\nu}}\left(\mathcal{G}_{\sigma}\right)_{0}+\sum_{\sigma \in \mathbb{Z}^{\nu}} \sum_{r \in J_{m}}\left(\mathcal{V}_{\sigma}^{r}\right)_{0}+\mathcal{D}+\mathcal{Z} & \text { if } \alpha=0, \\ \sum_{\sigma \in \mathbb{Z}^{\nu}}\left(\mathcal{G}_{\sigma}\right)_{\alpha}+\sum_{\sigma \in \mathbb{Z}^{\nu}} \sum_{r \in J_{m}}\left(\mathcal{V}_{\sigma}^{r}\right)_{\alpha} & \text { if } \alpha \in R_{\mathrm{sh}}, \\ \sum_{\sigma \in \mathbb{Z}^{\nu}}\left(\mathcal{G}_{\sigma}\right)_{\alpha} & \text { if } \alpha \in R_{\lg } .\end{cases}
$$


Now we note that as $\tilde{\mathcal{L}}$ is an $R$-graded Lie algebra, so is $\tilde{\mathcal{L}} / Z(\tilde{\mathcal{L}})$, and as $Z(\tilde{\mathcal{L}}) \subseteq$ $\mathcal{D}+\mathcal{Z}$, one can identify $\tilde{\mathcal{L}} / Z(\tilde{\mathcal{L}})$ as

$$
\tilde{\mathcal{L}} / Z(\tilde{\mathcal{L}})=\sum_{\sigma \in \mathbb{Z}^{\nu}} \mathcal{G}_{\sigma}+\sum_{\sigma \in \mathbb{Z}^{\nu}} \sum_{r \in J_{m}} \mathcal{V}_{\sigma}^{r}+\mathcal{D}^{\prime} \quad \text { where } \quad \mathcal{D}^{\prime}:=\mathcal{D} / Z(\tilde{\mathcal{L}})
$$

We shall keep the same notation for the images of $e_{i}, f_{i}, h_{i}, h_{i, j}^{ \pm}$and $v^{r}$ in $\tilde{\mathcal{L}} / Z(\tilde{\mathcal{L}})$ and use $[\cdot, \cdot]^{-}$for the Lie bracket on $\tilde{\mathcal{L}} / Z(\tilde{\mathcal{L}})$. Now using $3.8,2.9$ and Theorem 2.3, we have an epimorphism

$$
\begin{aligned}
& \psi^{\prime}: \tilde{\mathcal{L}} / Z(\tilde{\mathcal{L}}) \rightarrow \mathfrak{A} / Z(\mathfrak{A})=\left(\mathcal{G} \otimes A_{[\nu]}\right) \oplus\left(\mathcal{V} \otimes A_{[\nu]}^{m}\right) \oplus \mathcal{D}_{A_{[\nu]}^{m}, A_{[\nu]}^{m}} \\
& e_{i} \mapsto e_{i} \otimes 1, f_{i} \mapsto f_{i} \otimes 1, h_{i} \mapsto h_{i} \otimes 1, h_{i, j}^{ \pm} \mapsto h_{i} \otimes t_{j}^{ \pm 1}, v^{r} \mapsto v_{n_{s}} \otimes w_{r}
\end{aligned}
$$

for $i \in J_{\ell}, j \in J_{\nu}$ and $r \in J_{m}$. Next let $\alpha, \beta, \alpha+\beta \in R^{\times}, \gamma, \alpha+\gamma \in R_{\mathrm{sh}}, \sigma, \tau \in \mathbb{Z}^{\nu}$ and $r \in J_{m}$. It follows using Proposition 3.3 and 2.5 that $\psi^{\prime}\left(\left(\mathcal{G}_{\sigma}\right)_{\alpha}\right)=\mathcal{G}_{\alpha} \otimes t^{\sigma}$ and $\psi^{\prime}\left(\left(\mathcal{V}_{\sigma}^{r}\right)_{\gamma}\right)=\mathcal{V}_{\gamma} \otimes t^{\sigma} w_{r}$. Therefore, by 2.5 , we have

$$
\begin{aligned}
& \psi^{\prime}\left(\left[\left(\mathcal{G}_{\sigma}\right)_{\alpha},\left(\mathcal{G}_{\tau}\right)_{\beta}\right]^{-}\right)=\left[\psi^{\prime}\left(\left(\mathcal{G}_{\sigma}\right)_{\alpha}\right), \psi^{\prime}\left(\left(\mathcal{G}_{\tau}\right)_{\beta}\right)\right]=\mathcal{G}_{\alpha+\beta} \otimes t^{\sigma+\tau} \neq\{0\} \\
& \psi^{\prime}\left(\left[\left(\mathcal{G}_{\sigma}\right)_{\alpha},\left(\mathcal{V}_{\tau}^{r}\right)_{\gamma}\right]^{-}\right)=\left[\psi^{\prime}\left(\left(\mathcal{G}_{\sigma}\right)_{\alpha}\right), \psi^{\prime}\left(\left(\mathcal{V}_{\tau}^{r}\right)_{\gamma}\right)\right]=\mathcal{V}_{\alpha+\gamma} \otimes t^{\sigma+\tau} w_{r} \neq\{0\} \\
& \psi^{\prime}\left(\left[\left(\mathcal{V}_{\eta}^{s}\right)_{\zeta},\left(\mathcal{V}_{\tau}^{r}\right)_{\gamma}\right]^{-}\right)=\left[\psi^{\prime}\left(\left(\mathcal{V}_{\eta}^{s}\right)_{\zeta}\right), \psi^{\prime}\left(\left(\mathcal{V}_{\tau}^{r}\right)_{\gamma}\right)\right]=\left[\mathcal{V}_{\eta} \otimes t^{\zeta} w_{s}, \mathcal{V}_{\tau} \otimes t^{\gamma} w_{r}\right] .
\end{aligned}
$$

Step 2 (Simply laced types). We define a $\mathbb{Z}^{\nu}$-grading on $\tilde{\mathcal{L}}$ as follows. Recalling (3.12, we set

$$
\operatorname{deg}\left(e_{i}\right)=\operatorname{deg}\left(f_{i}\right)=\operatorname{deg}\left(h_{i}\right):=0, \quad \operatorname{deg}\left(h_{i, a}^{ \pm}\right):= \pm 2 \sigma_{a}
$$

and note that this defines a $\mathbb{Z}^{\nu}$-grading on the free Lie algebra generated by the set $\left\{e_{i}, f_{i}, h_{i}, h_{i, a}^{ \pm} \mid i \in J_{\ell}, a \in J_{\nu}\right\}$. As the relations (R1)-(R9) are generated by homogeneous elements, the above grading induces a grading on $\tilde{\mathcal{L}}$ which naturally defines a grading on $\tilde{\mathcal{L}} / Z(\tilde{\mathcal{L}})$. We set

$$
(\tilde{\mathcal{L}} / Z(\tilde{\mathcal{L}}))_{\alpha}^{\sigma}:=(\tilde{\mathcal{L}} / Z(\tilde{\mathcal{L}}))_{\alpha} \cap(\tilde{\mathcal{L}} / Z(\tilde{\mathcal{L}}))^{\sigma} ; \quad \alpha \in R, \sigma \in \mathbb{Z}^{\nu}
$$

$\mathrm{SO}$

$$
(\tilde{\mathcal{L}} / Z(\tilde{\mathcal{L}}))_{\alpha}^{\sigma}:=\left(\mathcal{G}_{\sigma}\right)_{\alpha} ; \quad \alpha \in R, \sigma \in \mathbb{Z}^{\nu}
$$

We next note that as $\tilde{\mathcal{L}} / Z(\tilde{\mathcal{L}})$ is a centerless $R$-graded Lie algebra, by the Recognition Theorem there exists a unital commutative associative algebra $A$ such that as a Lie algebra $\tilde{\mathcal{L}} / Z(\tilde{\mathcal{L}})$ can be identified with $\mathcal{G} \otimes A$. Considering this identification we have

$$
(\tilde{\mathcal{L}} / Z(\tilde{\mathcal{L}}))_{\alpha}=\mathcal{G}_{\alpha} \otimes A ; \quad \alpha \in R^{\times}
$$


Using the same argument as in [You, Subsection 2.6], one can identify $A$ with $A_{[\nu]}$ in such a way that $h_{i, a}^{ \pm}$is identified with $h_{i} \otimes t_{a}^{ \pm 1}$. This in particular implies that $\psi^{\prime}$ (see $4.2)$ is an isomorphism from $\tilde{\mathcal{L}} / Z(\tilde{\mathcal{L}})$ to $\mathfrak{A} / Z(\mathfrak{A})$. Now taking $\pi_{1}: \tilde{\mathcal{L}} \rightarrow \tilde{\mathcal{L}} / Z(\tilde{\mathcal{L}})$ and $\pi_{2}: \mathfrak{A} \rightarrow \mathfrak{A} / Z(\mathfrak{A})$ to be the natural projection maps and considering (3.8), we see that $\psi^{\prime-1} \circ \pi_{2} \circ \psi=\pi_{1}$. We conclude that $\psi: \tilde{\mathcal{L}} \rightarrow \mathfrak{A}$ is a central extension of $\mathfrak{A}$, but $\tilde{\mathcal{L}}$ is perfect, so by [MP, Proposition I.9.3], we conclude that $\psi: \tilde{\mathcal{L}} \rightarrow \mathfrak{A}$ is an isomorphism and so $\tilde{\mathcal{L}} \cong \mathfrak{A}$.

Step 3 (Type $G_{2}$ ). We recall that for type $G_{2}$, there is $0 \leq p \leq 3$ such that the corresponding pair of the $R$-graded Lie algebra $\mathfrak{A} / Z(\mathfrak{A})$ is $(S, L)$ where $S:=$ $\mathbb{Z} \sigma_{1} \oplus \cdots \oplus \mathbb{Z} \sigma_{\nu}, L:=3 \mathbb{Z} \sigma_{1} \oplus \cdots \oplus 3 \mathbb{Z} \sigma_{p} \oplus \mathbb{Z} \sigma_{p+1} \oplus \cdots \oplus \mathbb{Z} \sigma_{\nu}$, and that $m=3^{p}-1$. For $r \in J_{m}$, define

$$
\sigma^{r}:=\sum_{i=1}^{p} s_{i} \sigma_{i} \quad \text { where } \quad r=\sum_{i=1}^{p} 3^{i-1} s_{i} \quad \text { for } s_{1}, s_{2}, s_{3} \in\{0,1,2\} .
$$

Let $\sigma=\left(n_{1}, \ldots, n_{\nu}\right) \in \mathbb{Z}^{\nu}$, for $1 \leq i \leq p$, suppose that $m_{i}, r_{i} \in \mathbb{Z}$ are such that $r_{i} \in\{0,1,2\}$ and $n_{i}=3 m_{i}+r_{i}$, and set

$$
\begin{aligned}
& r_{\sigma}:=\sum_{i=1}^{p} 3^{i-1} r_{i}, \quad \delta_{\sigma}:=\sum_{i=1}^{p} m_{i} \sigma_{i}+\sum_{i=p+1}^{\nu} n_{i} \sigma_{i}, \\
& \theta_{\sigma}:=\delta_{\sigma}+\sigma^{r_{\sigma}}=\delta_{\sigma}+\sum_{i=1}^{p} r_{i} \sigma_{i} .
\end{aligned}
$$

Note that $\delta_{\sigma}=\theta_{\sigma}$ if $\sigma \in L$. Now we would like to define a $\mathbb{Z}^{\nu}$-grading on $\tilde{\mathcal{L}}$. For this, we recall (3.12), (4.7) and set, for $i \in J_{\ell}, a \in J_{p}, p+1 \leq b \leq \nu$ and $r \in J_{m}$,

$$
\begin{aligned}
\operatorname{deg}\left(e_{i}\right)= & \operatorname{deg}\left(f_{i}\right)=\operatorname{deg}\left(h_{i}\right):=0, \quad \operatorname{deg}\left(h_{i, a}^{ \pm}\right):= \pm 3 \sigma_{a}, \\
& \operatorname{deg}\left(h_{i, b}^{ \pm}\right):= \pm \sigma_{b}, \quad \operatorname{deg}\left(v^{r}\right):=\sigma^{r} .
\end{aligned}
$$

This defines a $\mathbb{Z}^{\nu}$-grading on the free Lie algebra generated by

$$
\left\{e_{i}, f_{i}, h_{i}, h_{i, a}^{ \pm}, v^{r} \mid i \in J_{\ell}, a \in J_{\nu}, r \in J_{m}\right\} .
$$

As before relations $(\mathrm{R} 1)-(\mathrm{R} 9)$ are generated by homogeneous elements, so this grading is naturally transferred to $\tilde{\mathcal{L}}$ and also to $\tilde{\mathcal{L}} / Z(\tilde{\mathcal{L}})$. Next we note that if $\sigma=\left(n_{1}, \ldots, n_{\nu}\right) \in \mathbb{Z}^{\nu}$ and $r \in J_{m}$ with $\sigma^{r}=\sum_{i=1}^{p} s_{i} \sigma_{i}$, then

$$
\begin{aligned}
& \mathcal{G}_{\sigma} \text { is homogeneous of degree } \sum_{i=1}^{p} 3 n_{i} \sigma_{i}+\sum_{i=p+1}^{\nu} n_{i} \sigma_{i} \\
& \mathcal{V}_{\sigma}^{r} \text { is homogeneous of degree } \sum_{i=1}^{p}\left(3 n_{i}+s_{i}\right) \sigma_{i}+\sum_{i=p+1}^{\nu} n_{i} \sigma_{i} .
\end{aligned}
$$


As before set

$$
(\tilde{\mathcal{L}} / Z(\tilde{\mathcal{L}}))_{\alpha}^{\sigma}:=(\tilde{\mathcal{L}} / Z(\tilde{\mathcal{L}}))_{\alpha} \cap(\tilde{\mathcal{L}} / Z(\tilde{\mathcal{L}}))^{\sigma} ; \quad \alpha \in R, \sigma \in \mathbb{Z}^{\nu}
$$

Since $\tilde{\mathcal{L}}$ is a $G_{2}$-graded Lie algebra, the Recognition Theorem states that there are a unital commutative associative algebra $A$ and a unital Jordan algebra $\mathcal{J}$ over $A$ having a normalized trace satisfying the $\mathrm{ch}_{3}$-identity (see Theorem 2.1) such that $\tilde{\mathcal{L}}$ is centrally isogenous with

$$
(\mathcal{G} \otimes A) \oplus\left(\mathcal{V} \otimes \mathcal{J}_{0}\right) \oplus D_{\mathcal{J}, \mathcal{J}}
$$

where $\mathcal{V}=\mathfrak{C}_{0}($ see $\S 2.1 .2)$. Next set $B:=\mathcal{J}_{0}$ and note that $D_{\mathcal{J}, \mathcal{J}}=D_{\mathcal{J}_{0}, \mathcal{J}_{0}}$. Now we have

$$
(\tilde{\mathcal{L}} / Z(\tilde{\mathcal{L}}))_{\alpha}= \begin{cases}\left(\mathcal{G}_{\alpha} \otimes A\right) \oplus\left(\mathcal{V}_{\alpha} \otimes B\right) & \text { if } \alpha \in R_{\mathrm{sh}}, \\ \mathcal{G}_{\alpha} \otimes A & \text { if } \alpha \in R_{\mathrm{lg}}\end{cases}
$$

On the other hand, using 4.1), 4.8, 4.6 and (4.7), we have, for $\sigma \in \mathbb{Z}^{\nu}$ and $\alpha \in R^{\times}$,

$$
(\tilde{\mathcal{L}} / Z(\tilde{\mathcal{L}}))_{\alpha}^{\sigma}= \begin{cases}\left(\mathcal{V}_{\delta_{\sigma}}^{r_{\sigma}}\right)_{\alpha} & \text { if } \alpha \in R_{\mathrm{sh}} \text { and } \sigma \in S \backslash L, \\ \left(\mathcal{G}_{\delta_{\sigma}}\right)_{\alpha} & \text { if } \alpha \in R^{\times} \text {and } \sigma \in L .\end{cases}
$$

Next let $\alpha \in R_{\lg }$ and $\sigma \in L$. Then using 4.9 and the one-dimensionality of $(\tilde{\mathcal{L}} / Z(\tilde{\mathcal{L}}))_{\alpha}^{\sigma}$ and $\mathcal{G}_{\alpha}$ we find that there is a one-dimensional subspace $A_{\alpha}^{\delta_{\sigma}}$ of $A$ such that

$$
(\tilde{\mathcal{L}} / Z(\tilde{\mathcal{L}}))_{\alpha}^{\sigma}=\mathcal{G}_{\alpha} \otimes A_{\alpha}^{\delta_{\sigma}}
$$

Using the same argument as for simply laced types, we see that $A_{\alpha}^{\delta_{\sigma}}=A_{\beta}^{\delta_{\sigma}}$ for all $\alpha, \beta \in R_{\lg }$ and $\sigma \in L$. Set

$$
A^{\delta_{\sigma}}:=A_{\alpha}^{\delta_{\sigma}} \quad \text { for } \sigma \in L \text { and any choice of } \alpha \in R_{\mathrm{lg}} .
$$

Now let $\sigma \in L$ and $\alpha$ be a short root, and take $\beta$ to be a long root such that $\alpha-\beta \in R^{\times}$. Then recalling 2.5 , we have

$$
\begin{aligned}
(\tilde{\mathcal{L}} / Z(\tilde{\mathcal{L}}))_{\alpha}^{\sigma} & =\left(\mathcal{G}_{\delta_{\sigma}}\right)_{\alpha}=\varphi_{\delta_{\sigma}}\left(\left[\mathcal{G}_{\alpha-\beta}, \mathcal{G}_{\beta}\right]\right)=\left[\mathcal{G}_{\alpha-\beta}, \varphi_{\delta_{\sigma}}\left(\mathcal{G}_{\beta}\right)\right] \\
& =\left[\mathcal{G}_{\alpha-\beta},\left(\mathcal{G}_{\delta_{\sigma}}\right)_{\beta}\right]=\left[\mathcal{G}_{\alpha-\beta},\left(\mathcal{G}_{\delta_{\sigma}}\right)_{\beta}\right]^{-}=\left[\mathcal{G}_{\alpha-\beta}, \mathcal{G}_{\beta} \otimes A^{\delta_{\sigma}}\right]^{-}=\mathcal{G}_{\alpha} \otimes A^{\delta_{\sigma}} .
\end{aligned}
$$

Therefore

$$
(\tilde{\mathcal{L}} / Z(\tilde{\mathcal{L}}))_{\alpha}^{\sigma}=\mathcal{G}_{\alpha} \otimes A^{\delta_{\sigma}} ; \quad \alpha \in R^{\times}, \sigma \in L
$$

Next suppose that $\alpha \in R_{\text {sh }}$ and $\sigma \in S \backslash L$. Then the one-dimensionality of $(\tilde{\mathcal{L}} / Z(\tilde{\mathcal{L}}))_{\alpha}^{\sigma}$ and $\mathcal{V}_{\alpha}$ together with 4.9 and 4.10 implies that there is a one- 
dimensional subspace $B_{\alpha}^{\theta_{\sigma}}$ such that

$$
(\tilde{\mathcal{L}} / Z(\tilde{\mathcal{L}}))_{\alpha}^{\sigma}=\mathcal{V}_{\alpha} \otimes B_{\alpha}^{\theta_{\sigma}}
$$

If $\sigma \in S \backslash L$ and $\alpha, \beta \in R_{\text {sh }}$ are such that $\alpha-\beta \in R^{\times}$, then we have

$$
\begin{aligned}
\mathcal{V}_{\alpha} \otimes B_{\alpha}^{\theta_{\sigma}} & =(\tilde{\mathcal{L}} / Z(\tilde{\mathcal{L}}))_{\alpha}^{\sigma}=\left(\mathcal{V}_{\delta_{\sigma}}^{r_{\sigma}}\right)_{\alpha}=\psi_{\delta_{\sigma}}^{r_{\sigma}}\left(\mathcal{G}_{\alpha-\beta} \cdot \mathcal{V}_{\beta}\right)=\left[\mathcal{G}_{\alpha-\beta}, \psi_{\delta_{\sigma}}^{r_{\sigma}}\left(\mathcal{V}_{\beta}\right)\right] \\
& =\left[\mathcal{G}_{\alpha-\beta},\left(\mathcal{V}_{\delta_{\sigma}}^{r_{\sigma}}\right)_{\beta}\right]=\left[\mathcal{G}_{\alpha-\beta}, \mathcal{V}_{\beta} \otimes B_{\beta}^{\theta_{\sigma}}\right]^{-}=\mathcal{G}_{\alpha-\beta} \cdot \mathcal{V}_{\beta} \otimes B_{\beta}^{\theta_{\sigma}}=\mathcal{V}_{\alpha} \otimes B_{\beta}^{\theta_{\sigma}}
\end{aligned}
$$

which implies that $B_{\alpha}^{\theta_{\sigma}}=B_{\beta}^{\theta_{\sigma}}$. Use [AG, (5.11)] to conclude that $B_{\alpha}^{\theta_{\sigma}}=B_{\beta}^{\theta_{\sigma}}$ for all $\alpha, \beta \in R_{\text {sh }}$ and define

$$
B^{\theta_{\sigma}}:=B_{\alpha}^{\theta_{\sigma}} \quad \text { for } \sigma \in S \backslash L \text { and any choice of } \alpha \in R_{\mathrm{sh}} .
$$

Now take $\sigma, \tau \in L$ and $\alpha, \beta \in R^{\times}$such that $\alpha+\beta \in R^{\times}$. Then (4.3), 4.11, (2.5) and Remark 2.1.2 imply that

$$
\begin{gathered}
0 \neq\left[(\tilde{\mathcal{L}} / Z(\tilde{\mathcal{L}}))_{\alpha}^{\sigma},(\tilde{\mathcal{L}} / Z(\tilde{\mathcal{L}}))_{\beta}^{\tau}\right]^{-} \subseteq(\tilde{\mathcal{L}} / Z(\tilde{\mathcal{L}}))_{\alpha+\beta}^{\sigma+\tau}=\mathcal{G}_{\alpha+\beta} \otimes A^{\delta_{\sigma+\tau}} \\
{\left[(\tilde{\mathcal{L}} / Z(\tilde{\mathcal{L}}))_{\alpha}^{\sigma},(\tilde{\mathcal{L}} / Z(\tilde{\mathcal{L}}))_{\beta}^{\tau}\right]^{-}=\left[\mathcal{G}_{\alpha} \otimes A^{\delta_{\sigma}}, \mathcal{G}_{\beta} \otimes A^{\delta_{\tau}}\right]^{-} \subseteq \mathcal{G}_{\alpha+\beta} \otimes A^{\delta_{\sigma}} A^{\delta_{\tau}}}
\end{gathered}
$$

Also for $\sigma \in L, \tau \in S \backslash L, \alpha \in R_{\lg }$ and $\beta \in R_{\text {sh }}$ such that $\alpha+\beta \in R_{\text {sh }}, 4.3$, 4.12, 4.11, 2.5 and Remark 2.1.2 imply that

$$
\begin{gathered}
0 \neq\left[(\tilde{\mathcal{L}} / Z(\tilde{\mathcal{L}}))_{\alpha}^{\sigma},(\tilde{\mathcal{L}} / Z(\tilde{\mathcal{L}}))_{\beta}^{\tau}\right]^{-} \subseteq(\tilde{\mathcal{L}} / Z(\tilde{\mathcal{L}}))_{\alpha+\beta}^{\sigma+\tau}=\mathcal{V}_{\alpha+\beta} \otimes B^{\theta_{\sigma+\tau}}=\mathcal{V}_{\alpha+\beta} \otimes B^{\delta_{\sigma}+\theta_{\tau}}, \\
{\left[(\tilde{\mathcal{L}} / Z(\tilde{\mathcal{L}}))_{\alpha}^{\sigma},(\tilde{\mathcal{L}} / Z(\tilde{\mathcal{L}}))_{\beta}^{\tau}\right]^{-}=\left[\mathcal{G}_{\alpha} \otimes A^{\delta_{\sigma}}, \mathcal{V}_{\beta} \otimes B^{\theta_{\tau}}\right]^{-} \subseteq \mathcal{V}_{\alpha+\beta} \otimes A^{\delta_{\sigma}} \cdot B^{\theta_{\tau}}}
\end{gathered}
$$

Therefore the one-dimensionality of the subspaces appearing on the right hand sides implies that

$$
\begin{array}{lll}
\text { (i) } A^{\delta_{\sigma}} \cdot A^{\delta_{\tau}}=A^{\delta_{\sigma}+\delta_{\tau}}=A^{\delta_{\sigma+\tau}} ; & & \sigma, \tau \in L, \\
\text { (ii) } A^{\delta_{\sigma}} \cdot B^{\theta_{\tau}}=B^{\delta_{\sigma}+\theta_{\tau}}=B^{\theta_{\sigma+\tau}} ; & & \sigma \in L, \tau \in S \backslash L .
\end{array}
$$

One can see that there are short roots $\alpha, \beta$ such that $\alpha+\beta \in R_{\text {sh }}$ and there are $x \in \mathcal{V}_{\alpha}, y \in \mathcal{V}_{\beta}$ such that

$$
D_{x, y} \neq 0 \quad \text { and } \quad x * y \neq 0 .
$$

Now if $\sigma, \gamma \in S \backslash L$, considering (4.7) and using (2.5), 2.13) and (2.14), we have

$$
\left[\mathcal{V}_{\alpha} \otimes t^{\delta_{\sigma}} w_{r_{\sigma}}, \mathcal{V}_{\beta} \otimes t^{\delta_{\gamma}} w_{r_{\gamma}}\right] \neq 0
$$

and so 4.10 and 4.3 imply that $0 \neq\left[(\tilde{\mathcal{L}} / Z(\tilde{\mathcal{L}}))_{\alpha}^{\sigma},(\tilde{\mathcal{L}} / Z(\tilde{\mathcal{L}}))_{\beta}^{\gamma}\right]^{-} \subseteq(\tilde{\mathcal{L}} / Z(\tilde{\mathcal{L}}))_{\alpha+\beta}^{\sigma+\gamma}$ and so the one-dimensionality of the subspaces implies that

$$
\left[(\tilde{\mathcal{L}} / Z(\tilde{\mathcal{L}}))_{\alpha}^{\sigma},(\tilde{\mathcal{L}} / Z(\tilde{\mathcal{L}}))_{\beta}^{\gamma}\right]^{-}=(\tilde{\mathcal{L}} / Z(\tilde{\mathcal{L}}))_{\alpha+\beta}^{\sigma+\gamma} .
$$


This together with $4.10-4.13$ implies that

$$
\left[\mathcal{V}_{\alpha} \otimes B^{\theta_{\sigma}}, \mathcal{V}_{\beta} \otimes B^{\theta_{\gamma}}\right]= \begin{cases}\mathcal{V}_{\alpha+\beta} \otimes B^{\theta_{\sigma+\gamma}} & \text { if } \sigma+\gamma \in S \backslash L \\ \mathcal{G}_{\alpha+\beta} \otimes A^{\delta_{\sigma+\gamma}} & \text { if } \sigma+\gamma \in L\end{cases}
$$

Now if $0 \neq a \in B^{\theta_{\sigma}}$ and $0 \neq b \in B^{\theta_{\gamma}}$, then $(\tilde{\mathcal{L}} / Z(\tilde{\mathcal{L}}))_{\alpha}^{\sigma}=\mathbb{C} x \otimes a$ and $(\tilde{\mathcal{L}} / Z(\tilde{\mathcal{L}}))_{\beta}^{\tau}=$ $\mathbb{C} y \otimes b$. Also using 2.5, we have

$$
[x \otimes a, y \otimes b]=D_{x, y} \otimes t(a, b)+(x * y) \otimes(a * b)+t(x, y) D_{a, b} .
$$

Therefore if $\sigma+\gamma \in L$, we have $0 \neq t(a, b) \in A^{\delta_{\sigma+\tau}}$ and $a * b=0$, which yields $a b=a * b+t(a, b) \in A^{\delta_{\sigma+\gamma}}=A^{\theta_{\sigma+\gamma}}$. Also if $\sigma+\gamma \in S \backslash L$, then $0 \neq a * b \in B^{\theta_{\sigma+\gamma}}$ and $t(a, b)=0$, which implies that $a b=a * b+t(a, b) \in B^{\theta_{\sigma+\gamma}}$. Summarizing, we have

$$
B^{\theta_{\sigma}} \cdot B^{\theta_{\gamma}}= \begin{cases}A^{\theta_{\sigma+\gamma}} & \text { if } \sigma+\gamma \in L \\ B^{\theta_{\sigma+\gamma}} & \text { if } \sigma+\gamma \in S \backslash L\end{cases}
$$

Next set

$$
\mathcal{J}^{\sigma}:= \begin{cases}A^{\theta_{\sigma}} & \text { if } \sigma \in L \\ B^{\theta_{\sigma}} & \text { if } \sigma \in S \backslash L .\end{cases}
$$

Now as $(\tilde{\mathcal{L}} / Z(\tilde{\mathcal{L}}))_{\alpha}=\sum_{\sigma \in \mathbb{Z}^{\nu}}(\tilde{\mathcal{L}} / Z(\tilde{\mathcal{L}}))_{\alpha}^{\sigma}, \alpha \in R^{\times}$, one can use 4.14 and 4.16 to deduce that $\mathcal{J}=A \oplus B=\bigoplus_{\sigma \in \mathbb{Z}^{\nu}} \mathcal{J}^{\sigma}$ is a graded Jordan algebra with onedimensional summands satisfying $\mathcal{J}^{\sigma} \cdot \mathcal{J}^{\tau}=\mathcal{J}^{\sigma+\tau}$. Therefore the same argument as in [AG, Proposition 5.58] shows that $A$ is graded isomorphic to $A_{[\nu]}$ and $\mathcal{J}$ is graded isomorphic to $\mathcal{J}_{p}$. Considering the Remark in 2.1 .2 and using the same argument as in [You, Subsection 2.6], we may identify $A$ with $A_{[\nu]}$ in such a way that

$$
h_{i, a}^{ \pm}=h_{i} \otimes t_{a}^{ \pm 1} ; \quad i \in J_{n}, j \in J_{\nu}
$$

Now let $r \in J_{m}=J_{p^{3}-1}$. Considering (4.7) and (4.6), one finds that $r_{\sigma^{r}}=r$ and $\delta_{\sigma^{r}}=0$, so $\mathcal{V}_{\delta_{\sigma^{r}}}^{r_{\sigma^{r}}}=\mathcal{V}_{0}^{r_{\sigma^{r}}}=\mathcal{V}^{r}$ and $\theta_{\sigma^{r}}=\sigma^{r}$. Therefore using 4.10, 4.12, 4.13 and 3.19, we have

$$
\begin{aligned}
\left(\mathcal{V}^{r}\right)_{n_{s}} & =\left(\mathcal{V}_{0}^{r_{\sigma^{r}}}\right)_{n_{s}}=\left(\mathcal{V}_{\delta_{\sigma^{r}}}^{r_{\sigma}}\right)_{n_{s}}=(\tilde{\mathcal{L}} / Z(\tilde{\mathcal{L}}))_{\alpha_{n_{s}}}^{\sigma^{r}}=\mathcal{V}_{\alpha_{n_{s}}} \otimes B^{\theta_{\sigma^{r}}} \\
& =\mathcal{V}_{\alpha_{n_{s}}} \otimes B^{\sigma^{r}}
\end{aligned}
$$

Thus considering 2.11), for each $r \in J_{m}$ one finds $\beta^{r} \in B^{\sigma^{r}}$ such that $v^{r}=$ $v_{n_{s}} \otimes \beta^{r}$. Since $\mathcal{V}$, as a $\mathcal{G}$-module, is generated by $v_{n_{s}}, 2.4$ implies that

$$
v_{ \pm i}^{r}=v_{ \pm i} \otimes \beta^{r} ; \quad n_{\ell}+1 \leq i \leq n_{s}, r \in J_{m} .
$$


Now one can see that there is $n_{\ell}+1 \leq i \leq n_{s}-1$ such that $v_{n_{s}} *_{-i} \neq 0$ and $D_{v_{n_{s}}, v_{-i}} \neq 0$. Using (R9), we find for $r, s \in J_{m}$ that

$$
\left[v^{r}, v_{-i}^{s}\right]=m_{-i}^{\prime} a_{r, s}^{\prime}\left[\frac{1}{2} \mathfrak{b}_{\sigma_{r, s}^{\prime}}^{t_{-i}^{\prime}}, e_{t_{-i}^{\prime}}\right]+m_{-i} a_{r, s}\left[\frac{1}{2} \mathfrak{b}_{\sigma_{r, s}}^{t_{i}}, v_{t_{i}}^{t_{r, s}}\right] .
$$

If $q_{1}:=\left|\sigma_{r, s}\right|$ and $q_{2}:=\left|\sigma_{r, s}^{\prime}\right|$, then there are $j_{1}, \ldots, j_{q_{1}}, i_{1}, \ldots, i_{q_{2}} \in J_{\nu}$ such that $t^{\sigma_{r, s}}=t_{j_{1}}^{ \pm 1} \cdots t_{j_{q_{1}}}^{ \pm 1}$ and $t^{\sigma_{r, s}^{\prime}}=t_{i_{1}}^{ \pm 1} \cdots t_{i_{q_{2}}}^{ \pm 1}$. Now 3.7), Convention 3.1. 44.17), (R4), 4.19, 2.5 and 2.15 imply that

$$
\begin{aligned}
m_{-i} a_{r, s}\left[\frac{1}{2} \mathfrak{b}_{\sigma_{r, s}}^{t_{i}}, v_{t_{i}}^{t_{r, s}}\right] & =m_{-i} a_{r, s}\left[\frac{1}{2} h_{t_{i}} \otimes t_{j_{q_{1}}}^{ \pm 1}, \ldots, \frac{1}{2} h_{t_{i}} \otimes t_{j_{1}}^{ \pm 1}, v_{t_{i}} \otimes \beta^{t_{r, s}}\right] \\
& =m_{-i} a_{r, s} v_{t_{i}} \otimes t_{j_{1}}^{ \pm 1} \ldots t_{j_{q_{1}}}^{ \pm 1} \beta^{t_{r, s}} \\
& =m_{-i} a_{r, s} v_{t_{i}} \otimes t^{\sigma_{r, s}} \beta^{t_{r, s}}=v_{n_{s}} * v_{-i} \otimes a_{r, s} t^{\sigma_{r, s}} \beta^{t_{r, s}}
\end{aligned}
$$

and

$$
\begin{aligned}
m_{-i}^{\prime} a_{r, s}^{\prime}\left[\frac{1}{2} \mathfrak{b}_{\sigma_{r, s}^{\prime}}^{t_{-i}^{\prime}}, e_{t_{-i}^{\prime}}\right] & =m_{-i}^{\prime} a_{r, s}^{\prime}\left[\frac{1}{2} h_{t_{-i}^{\prime}} \otimes t_{i_{q_{2}}}^{ \pm 1}, \ldots, \frac{1}{2} h_{t_{-i}^{\prime}} \otimes t_{i_{1}}^{ \pm 1}, e_{t_{-i}^{\prime}}^{\prime} \otimes 1\right] \\
& =m_{-i}^{\prime} a_{r, s}^{\prime} e_{t_{-i}^{\prime}} \otimes t_{i_{q_{2}}}^{ \pm 1} \ldots t_{i_{1}}^{ \pm 1}=d_{v_{n_{s}}, v_{-i}} \otimes a_{r, s}^{\prime} t^{\sigma_{r, s}^{\prime}} .
\end{aligned}
$$

Also 4.20-4.22 imply that

$$
\left[v^{r}, v_{-i}^{s}\right]=d_{v_{n_{s}}, v_{-i}} \otimes a_{r, s}^{\prime} t^{\sigma_{r, s}^{\prime}}+\left(v_{n_{s}} * v_{-i}\right) \otimes a_{r, s} t^{\sigma_{r, s}} \beta^{t_{r, s}} .
$$

On the other hand 4.19 together with 2.5 implies that

$$
\begin{aligned}
{\left[v^{r}, v_{-i}^{s}\right] } & =\left[v_{n_{s}} \otimes \beta^{r}, v_{-i} \otimes \beta^{s}\right] \\
& =d_{v_{n_{s}}, v_{-i}} \otimes t\left(\beta^{r}, \beta^{s}\right)+\left(v_{n_{s}} * v_{-i}\right) \otimes\left(\beta^{r} * \beta^{s}\right)+d_{\beta^{r}, \beta^{s}} .
\end{aligned}
$$

Altogether, these imply that

$$
t\left(\beta^{r}, \beta^{s}\right)=a_{r, s}^{\prime} t^{\sigma_{r, s}^{\prime}} \quad \text { and } \quad \beta^{r} * \beta^{s}=a_{r, s} t^{\sigma_{r, s}} \beta^{t_{r, s}} .
$$

Now 2.14) and (4.23) imply that we can identify $\beta^{r}$ with $w_{r}$ for $r \in J_{m}$. Therefore $\psi^{\prime}$ (see 4.2$)$ is an isomorphism from $\tilde{\mathcal{L}} / Z(\tilde{\mathcal{L}})$ to $\mathfrak{A} / Z(\mathfrak{A})$. Now considering 3.8 and using the same argument as for simply laced types, we conclude that $\psi: \tilde{\mathcal{L}} \rightarrow \mathfrak{A}$ is an isomorphism.

Step 3 (Type $F_{4}$ ). We recall that for type $F_{4}$, there is $0 \leq p \leq 3$ such that the corresponding pair of the $R$-graded Lie algebra $\mathfrak{A} / Z(\mathfrak{A})$ is $(S, L)$ where $S:=$ $\mathbb{Z} \sigma_{1} \oplus \cdots \oplus \mathbb{Z} \sigma_{\nu}$ and $L:=2 \mathbb{Z} \sigma_{1} \oplus \cdots \oplus 2 \mathbb{Z} \sigma_{p} \oplus \mathbb{Z} \sigma_{p+1} \oplus \cdots \oplus \mathbb{Z} \sigma_{\nu}$, and that $m=2^{p}-1$. For $r \in J_{m}$, define

$$
\sigma^{r}:=\sum_{i=1}^{p} s_{i} \sigma_{i} \quad \text { where } \quad r=\sum_{i=1}^{p} 2^{i-1} s_{i} \quad \text { for } s_{1}, s_{2}, s_{3} \in\{0,1\} .
$$


Let $\sigma=\left(n_{1}, \ldots, n_{\nu}\right) \in \mathbb{Z}^{\nu}$, for $1 \leq i \leq p$, suppose that $m_{i}, r_{i} \in \mathbb{Z}$ are such that $r_{i} \in\{0,1\}$ and $n_{i}=2 m_{i}+r_{i}$, and set

$$
\begin{aligned}
& r_{\sigma}:=\sum_{i=1}^{p} 2^{i-1} r_{i}, \quad \delta_{\sigma}:=\sum_{i=1}^{p} m_{i} \sigma_{i}+\sum_{i=p+1}^{\nu} n_{i} \sigma_{i}, \\
& \theta_{\sigma}:=\delta_{\sigma}+\sigma^{r_{\sigma}}=\delta_{\sigma}+\sum_{i=1}^{p} r_{i} \sigma_{i} .
\end{aligned}
$$

We mention that $\delta_{\sigma}=\theta_{\sigma}$ if $\sigma \in L$. Now we would like to define a $\mathbb{Z}^{\nu}$-grading on $\tilde{\mathcal{L}}$. For this, we note that for $i \in J_{\ell}, a \in J_{p}, p+1 \leq b \leq \nu$ and $r \in J_{m}$,

$$
\begin{aligned}
\operatorname{deg}\left(e_{i}\right)= & \operatorname{deg}\left(f_{i}\right)=\operatorname{deg}\left(h_{i}\right):=0, \quad \operatorname{deg}\left(h_{i, a}^{ \pm}\right):= \pm 2 \sigma_{a}, \\
& \operatorname{deg}\left(h_{i, b}^{ \pm}\right):= \pm \sigma_{b}, \quad \operatorname{deg}\left(v^{r}\right):=\sigma^{r},
\end{aligned}
$$

define a $\mathbb{Z}^{\nu}$-grading on the free Lie algebra generated by $\left\{e_{i}, f_{i}, h_{i}, h_{i, a}^{ \pm}, v^{r} \mid i \in J_{\ell}\right.$, $\left.a \in J_{\nu}, r \in J_{m}\right\}$. As before relations (R1)-(R9) are generated by homogeneous elements, so this grading is naturally transferred to $\tilde{\mathcal{L}}$ and also to $Z(\tilde{\mathcal{L}})$. Next we note that if $\sigma=\left(n_{1}, \ldots, n_{\nu}\right) \in \mathbb{Z}^{\nu}$, and if $i \in J_{p}, s_{i} \in\{0,1\}$ and $r:=\sum_{i=1}^{p} 2^{i-1} s_{i}$, then

$$
\begin{aligned}
& \mathcal{G}_{\sigma} \text { is homogeneous of degree } \sum_{i=1}^{p} 2 n_{i} \sigma_{i}+\sum_{i=p+1}^{\nu} n_{i} \sigma_{i} \\
& \mathcal{V}_{\sigma}^{r} \text { is homogeneous of degree } \sum_{i=1}^{p}\left(2 n_{i}+s_{i}\right) \sigma_{i}+\sum_{i=p+1}^{\nu} n_{i} \sigma_{i} .
\end{aligned}
$$

As before set

$$
(\tilde{\mathcal{L}} / Z(\tilde{\mathcal{L}}))_{\alpha}^{\sigma}:=(\tilde{\mathcal{L}} / Z(\tilde{\mathcal{L}}))_{\alpha} \cap(\tilde{\mathcal{L}} / Z(\tilde{\mathcal{L}}))^{\sigma} ; \quad \alpha \in R, \sigma \in \mathbb{Z}^{\nu}
$$

Since $\tilde{\mathcal{L}}$ is an $F_{4}$-graded Lie algebra, the Recognition Theorem states that there is a unital commutative associative algebra $A$ and an alternative algebra $\mathcal{C}$ over $A$ having a normalized trace $T$ satisfying the $\mathrm{ch}_{2}$-identity (see Theorem 2.1) such that $\tilde{\mathcal{L}}$ is centrally isogenous with

$$
(\mathcal{G} \otimes A) \oplus\left(\mathcal{V} \otimes \mathcal{C}_{0}\right) \oplus D_{\mathcal{C}, \mathcal{C}}
$$

where $\mathcal{V}=\mathfrak{J}_{0}($ see $\S 2.1 .2)$. Next set $B:=\mathcal{C}_{0}$ and note that $D_{\mathcal{C}, \mathcal{C}}=D_{\mathcal{C}_{0}, \mathcal{C}_{0}}$. Now we have

$$
(\tilde{\mathcal{L}} / Z(\tilde{\mathcal{L}}))_{\alpha}= \begin{cases}\left(\mathcal{G}_{\alpha} \otimes A\right) \oplus\left(\mathcal{V}_{\alpha} \otimes B\right) & \text { if } \alpha \in R_{\mathrm{sh}}, \\ \mathcal{G}_{\alpha} \otimes A & \text { if } \alpha \in R_{\mathrm{lg}} .\end{cases}
$$

Also for $\sigma \in \mathbb{Z}^{\nu}$ and $\alpha \in R^{\times}$by (4.26) and (4.1), we have

$$
(\tilde{\mathcal{L}} / Z(\tilde{\mathcal{L}}))_{\alpha}^{\sigma}= \begin{cases}\left(\mathcal{V}_{\delta_{\sigma}}^{r_{\sigma}}\right)_{\alpha} & \text { if } \alpha \in R_{\text {sh }} \text { and } \sigma \in S \backslash L, \\ \left(\mathcal{G}_{\delta_{\sigma}}\right)_{\alpha} & \text { if } \alpha \in R^{\times} \text {and } \sigma \in L .\end{cases}
$$


Using the same argument as for type $G_{2}$, one finds one-dimensional subspaces $A^{\delta_{\sigma}}, \sigma \in L$, and $B^{\theta_{\sigma}}, \sigma \in S \backslash L$, of $A$ and $B$ respectively satisfying

(i) $A^{\delta_{\sigma}} \cdot A^{\delta_{\tau}}=A^{\delta_{\sigma}+\delta_{\tau}}=A^{\delta_{\sigma+\tau}} ; \quad \sigma, \tau \in L$,

(ii) $A^{\delta_{\sigma}} \cdot B^{\theta_{\tau}}=B^{\delta_{\sigma}+\theta_{\tau}}=B^{\theta_{\sigma+\tau}} ; \quad \sigma \in L, \tau \in S \backslash L$,

(iii) $B^{\theta_{\sigma}} \cdot B^{\theta_{\tau}}= \begin{cases}A^{\theta_{\sigma+\tau}} & \text { if } \sigma, \tau \in S \backslash L, \sigma+\tau \in L, \\ B^{\theta_{\sigma+\tau}} & \text { if } \sigma, \tau \in S \backslash L, \sigma+\tau \in S \backslash L,\end{cases}$

such that

$$
(\tilde{\mathcal{L}} / Z(\tilde{\mathcal{L}}))_{\alpha}^{\sigma}= \begin{cases}\mathcal{G}_{\alpha} \otimes A^{\delta_{\sigma}} & \text { if } \alpha \in R^{\times}, \sigma \in L, \\ \mathcal{V}_{\alpha} \otimes B^{\theta_{\sigma}} & \text { if } \alpha \in R_{\mathrm{sh}}, \sigma \in S \backslash L .\end{cases}
$$

Now for $\sigma \in \mathbb{Z}^{\nu}$, set

$$
\mathcal{C}^{\sigma}:= \begin{cases}A^{\theta_{\sigma}} & \text { if } \sigma \in L, \\ B^{\theta_{\sigma}} & \text { if } \sigma \in S \backslash L .\end{cases}
$$

Then 4.16 implies that $\mathcal{C}:=\bigoplus_{\sigma \in \mathbb{Z}^{\nu}} \mathcal{C}^{\sigma}$ is a graded alternative algebra with onedimensional summands satisfying $\mathcal{C}^{\sigma} \cdot \mathcal{C}^{\tau}=\mathcal{C}^{\sigma+\tau}$. Therefore the same argument as in [AG, Proposition 5.45] shows that $A$ is graded isomorphic to $A_{[\nu]}$ and $\mathcal{C}$ is graded isomorphic to $\mathcal{A}_{p}$. Hence as before we may identify $A$ with $A_{[\nu]}$ such that

$$
h_{i, a}^{ \pm}=h_{i} \otimes t_{a}^{ \pm 1} ; \quad i \in J_{n}, j \in J_{\nu} .
$$

Considering (3.8) and using the same argument as for type $G_{2}$, one concludes that $\psi: \tilde{\mathcal{L}} \rightarrow \mathfrak{A}$ is an isomorphism.

\section{$\S 5$. Postponed proofs}

In this section we present the proofs of Propositions 3.4 and 3.5 . For this, we first need to prove the following claim about type $F_{4}$.

Claim. Suppose that $R$ is of type $F_{4}$. For $\sigma, \tau \in \mathbb{Z}^{\nu}, 1 \leq r, s \leq m$ with $r \neq s$, we have

(i) $\left[v_{\sigma, 3}^{s},\left[f_{3}, v_{\tau, 3}^{r}\right]\right]=0$.

(ii) $\left[v_{\sigma, 4}^{s},\left[f_{4}, v_{\tau, 4}^{r}\right]\right]=0$.

(iii) $\left[v_{\sigma, 3}^{s},\left[f_{4}, v_{\tau, 4}^{r}\right]\right]=-\left[v_{\sigma, 3}^{r},\left[f_{4}, v_{\tau, 4}^{s}\right]\right]$.

(iv) $\left[v_{\sigma, 4}^{s},\left[f_{3}, v_{\tau, 3}^{r}\right]\right]=-\left[v_{\sigma, 4}^{r},\left[f_{3}, v_{\tau, 3}^{s}\right]\right]$.

Proof of the Claim. (i) Knowing that $\varepsilon_{1}-\varepsilon_{4}$ is a positive long root, take $i \in J_{n}$ such that $\alpha_{i}=\varepsilon_{1}-\varepsilon_{4}$. Now as $-\alpha_{i}+\alpha_{n_{s}}=\varepsilon_{4}=\alpha_{3}$ (see (3.20), we have $\mathbb{C}\left[f_{i}, v_{\sigma}^{s}\right]=\mathbb{C} v_{\sigma, 3}^{s}$. Also as $\varepsilon_{1}-\varepsilon_{4}$ is not a short root, $\left[f_{3}, v_{\sigma}^{s}\right]=0$, and as $\alpha_{i}$ is 
a long root, $\left[f_{i}, f_{3}, v_{\tau, 3}^{r}\right]=0$. Therefore using the Jacobi identity together with Propositions 3.3, 3.1(iii) and (R9), we have

$$
\begin{aligned}
{\left[v_{\sigma, 3}^{s},\left[f_{3}, v_{\tau, 3}^{r}\right]\right] \in \mathbb{C}\left[\left[f_{i}, v_{\sigma}^{s}\right],\left[f_{3}, v_{\tau, 3}^{r}\right]\right] } & =\mathbb{C}\left(\left[f_{i}, v_{\sigma}^{s}, f_{3}, v_{\tau, 3}^{r}\right]-\left[v_{\sigma}^{s}, f_{i}, f_{3}, v_{\tau, 3}^{r}\right]\right) \\
& =\mathbb{C}\left[f_{i}, v_{\sigma}^{s}, f_{3}, v_{\tau, 3}^{r}\right]-0=\mathbb{C}\left[f_{i}, f_{3}, v_{\sigma}^{s}, v_{\tau, 3}^{r}\right] \\
& \subseteq \mathbb{C}[f_{i}, f_{3}, \underbrace{H, \ldots, H}_{|\sigma|+|\tau|}, v^{s}, v_{3}^{r}]=0 .
\end{aligned}
$$

(ii) We know that $\alpha_{4}=\frac{1}{2}\left(\varepsilon_{1}-\varepsilon_{2}-\varepsilon_{3}-\varepsilon_{4}\right)$. Take $j, t \in J_{n}$ such that $\alpha_{j}=$ $\frac{1}{2}\left(\varepsilon_{1}+\varepsilon_{2}+\varepsilon_{3}-\varepsilon_{4}\right)$ and $\alpha_{t}=\varepsilon_{2}+\varepsilon_{3}$, which are positive roots. Now we have

$$
\alpha_{4}+\alpha_{j}=\alpha_{i}=\varepsilon_{1}-\varepsilon_{4} \quad \text { and } \quad \alpha_{j}-\alpha_{4}=\alpha_{t}
$$

which are long roots, and so $\left[f_{t},\left[e_{4}, \mathcal{V}_{\tau,-4}^{r}\right]\right]=0$ and $\left[e_{4}, \mathcal{V}_{\sigma, j}^{s}\right]=0$. Now the Jacobi identity together with Propositions 3.3 and 3.1 (iii) implies that

$$
\begin{aligned}
{\left[v_{\sigma, 4}^{s},\left[f_{4}, v_{\tau, 4}^{r}\right]\right] } & \in\left[v_{\sigma, 4}^{s},\left[e_{4}, \mathcal{V}_{\tau,-4}^{r}\right]\right]=\left[\left[f_{t}, \mathcal{V}_{\sigma, j}^{s}\right],\left[e_{4}, \mathcal{V}_{\tau,-4}^{r}\right]\right] \\
& =\left[f_{t}, \mathcal{V}_{\sigma, j}^{s},\left[e_{4}, \mathcal{V}_{\tau,-4}^{r}\right]\right]+\left[\mathcal{V}_{\sigma, j}^{s}, f_{t},\left[e_{4}, \mathcal{V}_{\tau,-4}^{r}\right]\right] \\
& =\left[f_{t},\left[\mathcal{V}_{\sigma, j}^{s}, e_{4}\right], \mathcal{V}_{\tau,-4}^{r}\right]+\left[f_{t}, e_{4}, \mathcal{V}_{j}^{s}, \mathcal{V}_{\tau,-4}^{r}\right]=\left[f_{t}, e_{4}, \mathcal{V}_{\sigma, j}^{s}, \mathcal{V}_{\tau,-4}^{r}\right] \\
& \subseteq \mathbb{C}[f_{t}, e_{4}, \underbrace{H, \ldots, H}_{|\sigma|+|\tau|}, \mathcal{V}_{j}^{s}, \mathcal{V}_{-4}^{r}]=0
\end{aligned}
$$

(iii) Consider Lemma 2.1 and take $i, j \in J_{n}$ such that $\alpha_{i}=\varepsilon_{1}-\varepsilon_{4}$ and $-\alpha_{j}=\frac{1}{2}\left(-\varepsilon_{1}-\varepsilon_{2}-\varepsilon_{3}+\varepsilon_{4}\right)=-\alpha_{i}+\alpha_{4}$. Next we note that $-\alpha_{4}+\alpha_{3}$ is not a root and $\alpha_{i}+\alpha_{n_{s}}=\varepsilon_{4}=\alpha_{3}$. Therefore $\left[f_{4}, v_{\sigma, 3}^{s}\right]=0$ and $f_{i} \cdot v_{n_{s}}=z v_{3}$ for some $z \in \mathbb{C}$, which in turn implies that $z\left[f_{i}, v_{\sigma}^{s}\right]=v_{\sigma, 3}^{s}$. We next note that as $\alpha_{n_{s}} \neq \alpha_{j}$, there are $h_{1}, h_{2} \in \mathcal{H}$ such that $\alpha_{n_{s}}\left(h_{1}\right)=1=\alpha_{j}\left(h_{2}\right)$ and $\alpha_{n_{s}}\left(h_{2}\right)=0=\alpha_{j}\left(h_{1}\right)$. We take $c_{i}:=\left(h_{1}\right)^{i, \sigma}, c_{j}^{\prime}:=\left(h_{2}\right)^{j, \tau}$ for $1 \leq i \leq|\sigma|, 1 \leq j \leq|\tau|$ (see Convention 3.1). Also as $\alpha_{n_{s}}+\alpha_{4}$ is not a root, Propositions 3.3 and 3.1(iii) together with (R9) imply that $\left[v_{\sigma}^{s}, v_{\tau, 4}^{r}\right]=0$. So the Jacobi identity and Proposition 3.1 (iii) imply that

$$
\begin{aligned}
{\left[v_{\sigma, 3}^{s},\left[f_{4}, v_{\tau, 4}^{r}\right]\right] } & =\left[f_{4}, v_{\sigma, 3}^{s}, v_{\tau, 4}^{r}\right]=z\left[f_{4},\left[f_{i}, v_{\sigma}^{s}\right], v_{\tau, 4}^{r}\right] \\
& =z\left[f_{4}, f_{i},\left[v_{\sigma}^{s}, v_{\tau, 4}^{r}\right]\right]-z\left[f_{4}, v_{\sigma}^{s}, f_{i}, v_{\tau, 4}^{r}\right]=-z\left[f_{4}, v_{\sigma}^{s}, f_{i}, v_{\tau, 4}^{r}\right] \\
& =-z\left[f_{4}, c_{1}, \ldots, c_{|\sigma|}, c_{1}^{\prime}, \ldots, c_{|\tau|}^{\prime}, v^{s}, f_{i}, v_{4}^{r}\right] .
\end{aligned}
$$

Using (R4) together with the same argument as above, we conclude that

$$
\left[v_{\sigma, 3}^{r},\left[f_{4}, v_{\tau, 4}^{s}\right]\right]=-z\left[f_{4}, c_{1}, \ldots, c_{|\sigma|}, c_{1}^{\prime}, \ldots, c_{|\tau|}^{\prime}, v^{r}, f_{\gamma}, v_{2}^{s}\right]
$$

But in type $F_{4}, a_{r, s}=-a_{s, r}$ (see 2.14), so using (R9), we are done.

(iv) Use the same argument as in (i). 
We are now ready to prove Propositions 3.4 and 3.5 .

Proof of Proposition 3.4. (i) We prove the first equality, as the argument for the second is similar. We use induction on $|\tau|$. If $|\tau|=0$, then $e_{i, \tau}=e_{i}$, and $\left[e_{i}, e_{i, \sigma}\right]=$ $\varphi_{\sigma}\left(\left[e_{i}, e_{i}\right]\right)=0$. Now assume the result holds for all $\tau$ with $|\tau|=s$. Let $\sigma^{\prime}=$ $\left(m_{1}, \ldots, m_{\nu}\right)$ be such that $\left|\sigma^{\prime}\right|=s+1$. Then there is $a \in J_{\nu}$ such that $m_{a} \neq 0$. Set $\tau:=\sigma^{\prime}-\sigma_{a}^{\operatorname{sgn}\left(m_{a}\right)}$ and $\gamma:=\sigma+\sigma_{a}^{\operatorname{sgn}\left(m_{a}\right)}$ (see 3.12$)$ ). Then $|\tau|=\left|\sigma^{\prime}\right|-1=s$. Now Proposition 3.3 the Jacobi identity and (R4), (R6) together with the induction hypothesis give

$$
\begin{aligned}
{\left[e_{i, \sigma^{\prime}}, e_{i, \sigma}\right] } & =\left[\left[\frac{1}{2} h_{i, a}^{\operatorname{sgn}\left(m_{a}\right)}, e_{i, \tau}\right], e_{i, \sigma}\right]=\left[\frac{1}{2} h_{i, a}^{\operatorname{sgn}\left(m_{a}\right)}, e_{i, \tau}, e_{i, \sigma}\right]-\left[e_{i, \tau}, \frac{1}{2} h_{i, a}^{\operatorname{sgn}\left(m_{a}\right)}, e_{i, \sigma}\right] \\
& =\left[\frac{1}{2} h_{i, a}^{\operatorname{sgn}\left(m_{a}\right)}, e_{i, \tau}, e_{i, \sigma}\right]-\left[e_{i, \tau}, e_{i, \gamma}\right]=0-0=0 .
\end{aligned}
$$

(ii) Since $i \neq j$, there are $x, y \in \mathcal{H}$ such that

$$
\alpha_{j}(x)=1, \quad \alpha_{i}(x)=0, \quad \alpha_{j}(y)=0, \quad \alpha_{i}(y)=1 .
$$

Now using (3.16), the Jacobi identity and Propositions 3.3 and 3.1(iii),(ii), we have

$$
\begin{aligned}
{\left[e_{i, \sigma}, h_{j, \tau}\right] } & =-\left[e_{i, \sigma}, f_{j}, e_{j, \sigma}\right]=\left[f_{j}, e_{j, \sigma}, e_{i, \sigma}\right] \\
& =\left[f_{j},\left[x^{1, \tau}, \ldots, x^{|\tau|, \tau}, e_{j}\right],\left[y^{1, \sigma}, \ldots, y^{|\sigma|, \sigma}, e_{i}\right]\right] \\
& =\left[f_{j}, x^{1, \sigma}, \ldots, x^{|\sigma|, \sigma}, y^{1, \tau}, \ldots, y^{|\tau|, \tau}, e_{j}, e_{i}\right] \\
& =\left[f_{j}, y^{1, \sigma}, \ldots, y^{|\sigma|, \sigma}, y^{1, \tau}, \ldots, y^{|\tau|, \tau}, e_{j}, e_{i}\right] \\
& =\left[y^{1, \sigma}, \ldots, y^{|\sigma|, \sigma}, y^{1, \tau}, \ldots, y^{|\tau|, \tau}, f_{j}, e_{j}, e_{i}\right] \\
& =-\alpha_{i}\left(h_{j}\right)\left[y^{1, \sigma}, \ldots, y^{|\sigma|, \sigma}, y^{1, \tau}, \ldots, y^{|\tau|, \tau}, e_{i}\right]=-\alpha_{i}\left(h_{j}\right) e_{i, \tau+\sigma}
\end{aligned}
$$

Note that to get the last equality, we use (R6), (R4) (canceling relations). The second assertion is proved similarly.

(iii) We first note that by 3.16 , the Jacobi identity and (i),

$$
\left[e_{i, \tau}, h_{i, \sigma}\right]=\left[e_{i, \tau},\left[e_{i, \sigma}, f_{i}\right]\right]=[f_{i}, \overbrace{e_{i, \sigma}, e_{i, \tau}}^{0}]-\left[e_{i, \sigma}, \varphi_{\tau}\left[f_{i}, e_{i}\right]\right]=\left[e_{i, \sigma}, h_{i, \tau}\right] .
$$

Therefore using Proposition 3.3 , we have

$$
\left[h_{i, \tau}, e_{i, a}^{ \pm}\right]=\left[h_{i, a}^{ \pm}, e_{i, \tau}\right]=2 e_{i, \tau^{ \pm a}} .
$$

The second claim is proved similarly.

(iv) For $t \in J_{|\sigma|}$, using (R5), one finds that $\left(\alpha_{i}\left(h_{j}\right) / 2\right)\left[h_{i, a}^{ \pm}, f_{i}\right]=\left[h_{j, a}^{ \pm}, f_{i}\right]$ and $\left(\alpha_{i}\left(h_{j}\right) / 2\right)\left[h_{i, a}^{ \pm}, e_{i}\right]=\left[h_{j, a}^{ \pm}, e_{i}\right]$. Also by Proposition 3.3 , we get

$$
\left[\left(\frac{-1}{2} h_{i}\right)^{1, \sigma}, \ldots,\left(\frac{-1}{2} h_{i}\right)^{|\sigma|, \sigma}, f_{i}\right]=f_{i, \sigma} .
$$


Now these together with the Jacobi identity and (R4) imply that

$$
\begin{aligned}
{\left[h_{j, a}^{ \pm}, e_{i}, f_{i, \sigma}\right] } & =\left[e_{i}, h_{j, a}^{ \pm}, f_{i, \sigma}\right]+\left[\left[h_{j, a}^{ \pm}, e_{i}\right], f_{i, \sigma}\right] \\
& =\left[e_{i},\left(\frac{-1}{2} h_{i}\right)^{1, \sigma}, \ldots,\left(\frac{-1}{2} h_{i}\right)^{|\sigma|, \sigma}, h_{j, a}^{ \pm}, f_{i}\right]+\left[\left[h_{j, a}^{ \pm}, e_{i}\right], f_{i, \sigma}\right] \\
& =\frac{\alpha_{i}\left(h_{j}\right)}{2}\left(\left[e_{i},\left(\frac{-1}{2} h_{i}\right)^{1, \sigma}, \ldots,\left(\frac{-1}{2} h_{i}\right)^{|\sigma|, \sigma}, h_{i, a}^{ \pm}, f_{i}\right]+\left[\left[h_{i, a}^{ \pm}, e_{i}\right], f_{i, \sigma}\right]\right) \\
& =\frac{\alpha_{i}\left(h_{j}\right)}{2}\left(\left[e_{i}, h_{i, a}^{ \pm}, f_{i, \sigma}\right]+\left[\left[h_{i, a}^{ \pm}, e_{i}\right], f_{i, \sigma}\right]\right)=\frac{\alpha_{i}\left(h_{j}\right)}{2}\left[h_{i, a}^{ \pm}, e_{i}, f_{i, \sigma}\right] .
\end{aligned}
$$

Now we are done as by 3.16$),\left[e_{i}, f_{i, \sigma}\right]=h_{i, \sigma}$.

(v) Using Proposition 3.3 together with the Jacobi identity and 3.16, we have

$$
\begin{aligned}
{\left[e_{i, a}^{ \pm}, f_{i, \tau}\right] } & =\left[\left[\frac{1}{2} h_{i, a}^{ \pm}, e_{i}\right], f_{i, \tau}\right]=\left[\frac{1}{2} h_{i, a}^{ \pm}, e_{i}, f_{i, \tau}\right]-\left[e_{i}, \frac{1}{2} h_{i, a}^{ \pm}, f_{i, \tau}\right] \\
& =\left[\frac{1}{2} h_{i, a}^{ \pm}, h_{i, \tau}\right]+\left[e_{i}, f_{i, \tau^{ \pm}}\right]=\frac{1}{2}\left[h_{i, a}^{ \pm}, h_{i, \tau}\right]+h_{i, \tau^{ \pm} a} .
\end{aligned}
$$

(vi) We first note that as $\alpha_{n_{s}} \neq \alpha_{i}$, there are $x, y \in \mathcal{H}$ such that $\alpha_{n_{s}}(y)=0$, $\alpha_{i}(y)=1, \alpha_{n_{s}}(x)=1, \alpha_{i}(x)=0$ and so using Proposition 3.1(i), we have $\left[v^{r}, y^{i, \sigma}\right]=0, i \in J_{|\sigma|}$, which together with Proposition 3.3 implies that $\left[v^{r}, f_{i, \sigma}\right]=$ $\left[y^{1, \sigma}, \ldots, y^{|\sigma|, \sigma}, v^{r}, f_{i}\right]$. Now using this, 3.16 , the Jacobi identity, (R2), and Propositions 3.1(iii) and 3.3. we get

$$
\begin{aligned}
{\left[v^{r}, h_{i, \sigma}\right] } & =\left[v^{r}, e_{i}, f_{i, \sigma}\right]=\left[e_{i}, v^{r}, f_{i, \sigma}\right]=\left[x^{1, \sigma}, \ldots, x^{|\sigma|, \sigma}, e_{i}, v^{r}, f_{i}\right] \\
& =-\alpha_{n_{s}}\left(h_{i}\right)\left[x^{1, \sigma}, \ldots, x^{|\sigma|, \sigma}, v^{r}\right]=-\alpha_{n_{s}}\left(h_{i}\right) v_{\sigma}^{r} .
\end{aligned}
$$

This completes the proof of the first assertion.

Now we have $\left[v^{r}, h_{i, \sigma}\right]=-\alpha_{n_{s}}\left(h_{i}\right)\left[x^{1, \sigma}, \ldots, x^{|\sigma|, \sigma}, v^{r}\right]$ and $\left[v^{r}, h_{i, \tau}\right]=$ $-\alpha_{n_{s}}\left(h_{i}\right)\left[x^{1, \tau}, \ldots, x^{|\tau|, \tau}, v^{r}\right]$. Next we note that using 3.16 , Propositions 3.3 and 3.1 (i), the Jacobi identity and (R4), we find that $\left[h_{i, \tau}, x^{j, \sigma}\right]=0, j \in J_{|\sigma|}$, so

$$
\begin{aligned}
{\left[h_{i, \tau}, h_{i, \sigma}, v^{r}\right] } & =\alpha_{n_{s}}\left(h_{i}\right)\left[x^{1, \sigma}, \ldots, x^{|\sigma|, \sigma}, h_{i, \tau}, v^{r}\right] \\
& =\left(\alpha_{n_{s}}\left(h_{i}\right)\right)^{2}\left[x^{1, \sigma}, \ldots, x^{|\sigma|, \sigma}, x^{1, \tau}, \ldots, x^{|\tau|, \tau}, v^{r}\right] .
\end{aligned}
$$

Similarly $\left[h_{i, \tau}, h_{i, \sigma}, v^{r}\right]=\left(\alpha_{n_{s}}\left(h_{i}\right)\right)^{2}\left[x^{1, \sigma}, \ldots, x^{|\sigma|, \sigma}, x^{1, \tau}, \ldots, x^{|\tau|, \tau}, v^{r}\right]$. Now using (R4), we are done.

Proof of Proposition 3.5. (i) is immediate using Proposition 3.3 together with (R4), (R6).

(ii) We first suppose that $t \neq n_{s}$. Then there exists $x \in \mathcal{H}$ such that $\alpha_{n_{s}}(x)=0$ and $\alpha_{t}(x)=1$. Recall Convention 3.1, and set $c_{j}:=x^{j, \sigma}$ for $j \in J_{|\sigma|}$. Then using Propositions 3.3 and 3.1(i), we have

$$
\begin{aligned}
{\left[v^{r},\left(\mathcal{G}_{\sigma}\right)_{ \pm \alpha_{t}}\right] } & =\left[v^{r}, c_{1}, \ldots, c_{|\sigma|}, \mathcal{G}_{ \pm \alpha_{t}}\right]=\left[c_{1}, \ldots, c_{|\sigma|}, v^{r}, \mathcal{G}_{ \pm \alpha_{t}}\right] \\
& \subseteq\left[c_{1}, \ldots, c_{|\sigma|},\left(\mathcal{V}^{r}\right)_{ \pm \alpha_{t}+\alpha_{n_{s}}}\right]=\left(\mathcal{V}_{\sigma}^{r}\right)_{ \pm \alpha_{t}+\alpha_{n_{s}}} .
\end{aligned}
$$


Next let $t=n_{s}$. Then as $\alpha_{t}$ is not a simple root, there are $1 \leq j \leq \ell$ and $1 \leq i \leq n$ such that $\alpha_{t}-\alpha_{j}=\alpha_{i}$. Now $\left(\mathcal{G}_{\sigma}\right)_{\alpha_{t}}=\left[e_{j},\left(\mathcal{G}_{\sigma}\right)_{\alpha_{i}}\right]$ and so by (R2), the Jacobi identity and the first part of the proof, we have

$$
\left[v^{r},\left(\mathcal{G}_{\sigma}\right)_{\alpha_{t}}\right]=\left[v^{r}, e_{j},\left(\mathcal{G}_{\sigma}\right)_{\alpha_{i}}\right]=\left[e_{j}, v^{r},\left(\mathcal{G}_{\sigma}\right)_{\alpha_{i}}\right] \subseteq\left[e_{j},\left(\mathcal{V}_{\sigma}^{r}\right)_{\alpha_{i}+\alpha_{n_{s}}}\right] \subseteq\left(\mathcal{V}_{\sigma}^{r}\right)_{\alpha_{t}+\alpha_{n_{s}}} .
$$

Also as $\alpha_{t}=\alpha_{n_{s}}$ is a short root, Lemma 2.1 implies that there are $i, j \in J_{n}$ such that $\alpha_{t}+\alpha_{j}=\alpha_{i}$, so $\left(\mathcal{G}_{\sigma}\right)_{-\alpha_{t}}=\left[e_{j},\left(\mathcal{G}_{\sigma}\right)_{-\alpha_{i}}\right]$. Now as before we have

$$
\begin{aligned}
{\left[v^{r},\left(\mathcal{G}_{\sigma}\right)_{-\alpha_{t}}\right] } & =\left[v^{r}, e_{j},\left(\mathcal{G}_{\sigma}\right)_{-\alpha_{i}}\right]=\left[e_{j}, v^{r},\left(\mathcal{G}_{\sigma}\right)_{-\alpha_{i}}\right] \\
& \subseteq\left[e_{j},\left(\mathcal{V}_{\sigma}^{r}\right)_{-\alpha_{i}+\alpha_{n_{s}}}\right] \subseteq\left(\mathcal{V}_{\sigma}^{r}\right)_{-\alpha_{t}+\alpha_{n_{s}}} .
\end{aligned}
$$

Next using (R2), the Jacobi identity and the first part of the proof, we have

$$
\begin{aligned}
{\left[v^{r},\left(\mathcal{G}_{\sigma}\right)_{0}\right] } & =\sum_{i=1}^{\ell}\left[v^{r}, e_{i},\left(\mathcal{G}_{\sigma}\right)_{-\alpha_{i}}\right]=\sum_{i=1}^{\ell}\left[e_{i}, v^{r},\left(\mathcal{G}_{\sigma}\right)_{-\alpha_{i}}\right] \\
& \subseteq \sum_{i=1}^{\ell}\left[e_{i},\left(\mathcal{V}_{\sigma}^{r}\right)_{-\alpha_{i}+\alpha_{n_{s}}}\right] \subseteq\left(\mathcal{V}_{\sigma}^{r}\right)_{\alpha_{n_{s}}} .
\end{aligned}
$$

(iii) Using Lemma 2.2 (ii), one sees that $\left(\mathcal{V}_{\sigma}^{r}\right)_{0}$ is spanned by $\left[f_{i}, v_{\sigma, i}^{r}\right]$ for $n_{\ell}+1$ $\leq i \leq \ell$. So it is enough to show that there is a basis $\left\{x_{j} \in \mathcal{H} \mid j \in J_{\ell}\right\}$ of $\mathcal{H}$ such that $\left[\left(x_{j}\right)_{a}^{ \pm}, f_{i}, v_{\sigma, i}^{r}\right]=0$ for all $j \in J_{\ell}$ and $a \in J_{\nu}$. Fix $n_{\ell}+1 \leq i \leq \ell$. By Lemma 2.1(i), $\alpha_{i}=\beta+\gamma$ where $\beta$ is a short root and $\gamma$ is a long root. Take $t, t^{\prime} \in J_{n}$ such that $\beta= \pm \alpha_{t}$ and $\gamma= \pm \alpha_{t^{\prime}}$ and set

$$
e:=\left\{\begin{array}{ll}
f_{t^{\prime}} & \text { if } \gamma=\alpha_{t^{\prime}}, \\
e_{t^{\prime}} & \text { if } \gamma=-\alpha_{t^{\prime}},
\end{array} \quad f:=\left\{\begin{array}{ll}
f_{t} & \text { if } \beta=\alpha_{t}, \\
e_{t} & \text { if } \beta=-\alpha_{t},
\end{array} \quad v:= \begin{cases}v_{t}^{r} & \text { if } \beta=\alpha_{t}, \\
v_{-t}^{r} & \text { if } \beta=-\alpha_{t} .\end{cases}\right.\right.
$$

Then $e \in \mathcal{G}_{-\gamma}, f \in \mathcal{G}_{-\beta},[e, f] \in \mathcal{G}_{-\alpha_{i}}$ and $v \in\left(\mathcal{V}^{r}\right)_{\beta}$ and so we have

$$
\left[e, v_{\sigma, i}^{r}\right] \in \mathbb{C} \psi_{\sigma}^{r}(v) \text { and }\left[f, v_{\sigma, i}^{r}\right]=0 .
$$

Consider a basis $\left\{x_{i} \mid 1 \leq i \leq \ell-1\right\}$ for $\operatorname{ker}\left(\alpha_{i}\right)$. Also note that since $\alpha_{t^{\prime}} \neq \alpha_{i}$, there is $x_{\ell} \in \mathcal{H}$ such that $\alpha_{t}\left(x_{\ell}\right)=0$ and $\alpha_{i}\left(x_{\ell}\right) \neq 0$. Therefore $\left\{x_{i} \mid 1 \leq i \leq \ell\right\}$ is a basis for $\mathcal{H}$. Now we note, using Proposition 3.3 , that $v_{\sigma, i}^{r} \in[\underbrace{H, \ldots, H}_{|\sigma| \text { times }}, v_{i}^{r}]$ and $\psi_{\sigma}^{r}(v) \in[\underbrace{H, \ldots, H}_{|\sigma| \text { times }}, v]$. Using the Jacobi identity together with Proposition $3.1($ i), (R4) and 5.2), we have, for $1 \leq j^{\prime} \leq \ell-1$,

$$
\left[\left(x_{j^{\prime}}\right)_{a}^{ \pm}, f_{i}, v_{\sigma, i}^{r}\right] \in \mathbb{C}[f_{i},\left(x_{j^{\prime}}\right)_{a}^{ \pm}, \underbrace{H, \ldots, H}_{|\sigma| \text { times }}, v_{i}^{r}] \subseteq \mathbb{C}[f_{i}, \underbrace{H, \ldots, H}_{|\sigma| \text { times }},\left(x_{j^{\prime}}\right)_{a}^{ \pm}, v_{i}^{r}]=0,
$$


and

$$
\begin{aligned}
{\left[\left(x_{\ell}\right)_{a}^{ \pm}, f_{i}, v_{\sigma, i}^{r}\right] \in \mathbb{C}\left[\left(x_{\ell}\right)_{a}^{ \pm},[e, f], v_{\sigma, i}^{r}\right] } & \subseteq \mathbb{C}\left[\left(x_{\ell}\right)_{a}^{ \pm},\left[f, \psi_{\sigma}^{r}(v)\right]\right] \\
& \subseteq \mathbb{C}\left([f,\left(x_{\ell}\right)_{a}^{ \pm}, \underbrace{H, \ldots, H}_{|\sigma| \text { times }}, v]\right. \\
& \subseteq \mathbb{C}\left([f, \underbrace{H, \ldots, H}_{|\sigma| \text { times }},\left(x_{\ell}\right)_{a}^{ \pm}, v]=0 .\right.
\end{aligned}
$$

This completes the proof of this part.

(iv) follows from (iii) together with Proposition 3.3 , (R4) and (R7).

(v) Using Lemma 2.1(i), one finds $t, t^{\prime} \in J_{n}$ such that $\alpha_{t^{\prime}}$ is a short root, $\alpha_{t}$ is a long root and $\alpha_{n_{s}}= \pm \alpha_{t^{\prime}}+\alpha_{t}$. We note that $v_{\sigma}^{r}$ is a weight vector of weight $\alpha_{n_{s}}$ and so $v_{\sigma}^{r} \in \mathbb{C}\left[e_{t}, v_{\sigma, \pm t^{\prime}}^{r}\right]$. Now using (R2), we have

$$
\left[v^{s}, v_{\sigma}^{r}\right] \in \mathbb{C}\left[v^{s},\left[e_{t}, v_{\sigma, \pm t^{\prime}}^{r}\right]\right]=\left[\left[e_{t}, v^{s}\right], \mathcal{V}_{\sigma, \pm t^{\prime}}^{r}\right]+\left[e_{t}, v^{s}, v_{\sigma, \pm t^{\prime}}^{r}\right]=\left[e_{t}, v^{s}, v_{\sigma, \pm t^{\prime}}^{r}\right] .
$$

Since $t^{\prime} \neq n_{s}$, there is $h \in \mathcal{H}$ such that $\alpha_{t^{\prime}}(h)=1$ and $\alpha_{n_{s}}(h)=0$. For $i \in J_{|\sigma|}$ set $c_{i}:=h^{i, \sigma}$ (Convention 3.1. Now using Proposition 3.3. we have $\mathbb{C} v_{\sigma, \pm t^{\prime}}^{r}=\mathbb{C}\left[c_{1}, \ldots, c_{|\sigma|}, v_{ \pm t^{\prime}}^{r}\right]$ and so by Proposition 3.1(i),

$$
\left[v^{s}, v_{\sigma, \pm t^{\prime}}^{r}\right] \in \mathbb{C}\left[v^{s}, c_{1}, \ldots, c_{|\sigma|}, v_{ \pm t^{\prime}}^{r}\right]=\mathbb{C}\left[c_{1}, \ldots, c_{|\sigma|}, v^{s}, v_{ \pm t^{\prime}}^{r}\right] .
$$

As $t^{\prime} \neq n_{s}$ and $2 \alpha_{n_{s}}$ is not a root, we are done using (R9) together with Proposition 3.3 .

(vi) Suppose that $\alpha$ is a short root not equal to $\pm \alpha_{n_{s}}$. Then there is $n_{\ell}+1$ $\leq t \leq n_{s}-1$ such that $\alpha= \pm \alpha_{t}$. Since $\alpha \neq \alpha_{n_{s}}$, there is $h \in \mathcal{H}$ such that $\alpha(h)=1$ and $\alpha_{n_{s}}(h)=0$. Now using Proposition 3.3. one finds that $\left(\mathcal{V}_{\sigma}^{r}\right)_{ \pm \alpha_{t}}=$ $\mathbb{C}\left[h^{1, \sigma}, \ldots, h^{|\sigma|, \sigma}, v_{ \pm t}^{r}\right]$ and so Proposition 3.1(iii) implies that

$$
\left[v^{s},\left(\mathcal{V}_{\sigma}^{r}\right)_{ \pm \alpha_{t}}\right]=\mathbb{C}\left[v^{s}, h^{1, \sigma}, \ldots, h^{|\sigma|, \sigma}, v_{ \pm t}^{r}\right]=\mathbb{C}\left[h^{1, \sigma}, \ldots, h^{|\sigma|, \sigma}, v^{s}, v_{ \pm t}^{r}\right] .
$$

Hence we are done using (R9) together with Proposition 3.3 . Next suppose $\alpha=0$. By Lemma 2.2 (ii), $\left(\mathcal{V}_{\sigma}^{r}\right)_{0}$ is spanned by $\left[e_{i}, v_{\sigma,-i}^{r}\right], n_{\ell}+1 \leq i \leq \ell$. Fix $n_{\ell}+1 \leq i \leq \ell$. Then we have

$$
\left[v^{s},\left[e_{i}, v_{\sigma,-i}^{r}\right]\right]=\left[e_{i},\left[v^{s}, v_{\sigma,-i}^{r}\right]\right] .
$$

This together with the previous step completes the proof.

(vii) We first consider type $F_{4}$. Take $i, j, t^{\prime}, p, q \in J_{n}$ such that

$$
\begin{gathered}
\alpha_{i}=\frac{1}{2}\left(\varepsilon_{1}-\varepsilon_{2}-\varepsilon_{3}-\varepsilon_{4}\right), \quad \alpha_{j}=\varepsilon_{2}+\varepsilon_{3}, \quad \alpha_{t^{\prime}}=\frac{1}{2}\left(\varepsilon_{1}-\varepsilon_{2}-\varepsilon_{3}+\varepsilon_{4}\right), \\
\alpha_{p}=\frac{1}{2}\left(\varepsilon_{1}+\varepsilon_{2}+\varepsilon_{3}-\varepsilon_{4}\right), \quad \alpha_{q}=\frac{1}{2}\left(\varepsilon_{1}+\varepsilon_{2}+\varepsilon_{3}+\varepsilon_{4}\right) .
\end{gathered}
$$


Recalling $b, b^{\prime}$ from (3.21), one observes that there are non-zero scalars $a_{1}, a_{2}, a_{3}, a_{4}, y, z \in \mathbb{C}$ satisfying $\frac{-z a^{\prime}}{a_{2}}+\frac{y a}{a_{3} a_{1}} \neq 0$ and

$$
\begin{aligned}
& {\left[\left[f_{j}, f_{i}\right], v_{p}\right]=a_{1}\left[f_{4}, v_{4}\right], \quad\left[v_{n_{s}}, f_{n_{s}}\right]=a_{2}\left[v_{3}, f_{3}\right], \quad v_{n_{s}}=a_{3}\left[e_{t^{\prime}}, v_{p}\right],} \\
& {\left[f_{t^{\prime}}, f_{j}, f_{i}, b^{\prime}\left[f_{3}, v_{3}\right]+b\left[f_{4}, v_{4}\right]\right]=y v_{-n_{s}},} \\
& {\left[f_{n_{s}}, b^{\prime}\left[f_{3}, v_{3}\right]+b\left[f_{4}, v_{4}\right]\right]=z v_{-n_{s}} .}
\end{aligned}
$$

Now we recall that $\mathcal{V}^{s}$ and $\mathcal{V}_{\sigma}^{r}$ are $\mathcal{G}$-modules whose weights are short roots and note that $\alpha_{p}-\alpha_{i}, \alpha_{t^{\prime}}+\alpha_{q}$ are not short roots and $\alpha_{i}+\alpha_{q}=\varepsilon_{1}=\alpha_{n_{s}}$. So the Jacobi identity together with (vi) implies that

$$
\begin{aligned}
X:=y\left[e_{t^{\prime}}, v_{p}^{s}, v_{\sigma,-n_{s}}^{r}\right] & \in \mathbb{C}\left[e_{t^{\prime}}, v_{p}^{s}, f_{i}, v_{\sigma,-q}^{r}\right] \\
& =\mathbb{C}\left([e_{t^{\prime}}, \underbrace{\left.v_{p}^{s}, f_{i}\right]}_{0}, v_{\sigma,-q}^{r}]+\left[e_{t^{\prime}}, f_{i}, v_{p}^{s}, v_{\sigma,-q}^{r}\right]\right) \\
& =\mathbb{C}\left[e_{t^{\prime}}, f_{i},\left[f_{t^{\prime}}, v^{s}\right], v_{\sigma,-q}^{r}\right] \\
& \subseteq \mathbb{C}\left[e_{t^{\prime}}, f_{i}, f_{t^{\prime}}, v^{s}, v_{\sigma,-q}^{r}\right]+\mathbb{C}[e_{t^{\prime}}, f_{i}, v^{s}, \underbrace{f_{t^{\prime}}, v_{\sigma,-q}^{r}}_{0}] \\
& \subseteq\left[e_{t^{\prime}}, f_{i}, f_{t^{\prime}}, \sum_{\tau \in \mathbb{Z}^{\nu}}\left(\mathcal{G}_{\tau}\right)_{\alpha_{i}}+\sum_{1 \leq p \leq m} \sum_{\tau \in \mathbb{Z}^{\nu}}\left(\mathcal{V}_{\tau}^{p}\right)_{\alpha_{i}}\right] \\
& \subseteq \sum_{\tau \in \mathbb{Z}^{\nu}}\left(\mathcal{G}_{\tau}\right)_{0}+\sum_{1 \leq k \leq m} \sum_{\tau \in \mathbb{Z}^{\nu}}\left(\mathcal{V}_{\tau}^{k}\right)_{0} .
\end{aligned}
$$

Next we note that $\alpha_{j}+\alpha_{i}=\alpha_{p}=\alpha_{n_{s}}-\alpha_{t^{\prime}}$, so the Jacobi identity, Proposition 3.2 and (vi) imply that

$$
\begin{aligned}
{\left[\left[f_{j}, f_{i}\right], v_{p}^{s},\left(\mathcal{V}_{\sigma}^{r}\right)_{0}\right] } & \subseteq \mathbb{C}\left[f_{p}, v_{p}^{s},\left(\mathcal{V}_{\sigma}^{r}\right)_{0}\right] \subseteq \mathbb{C}\left[f_{p},\left[f_{t^{\prime}}, v^{s}\right],\left(\mathcal{V}_{\sigma}^{r}\right)_{0}\right] \\
& =\mathbb{C}\left(\left[f_{p}, f_{t^{\prime}}, v^{s},\left(\mathcal{V}_{\sigma}^{r}\right)_{0}\right]-\left[f_{p}, v^{s}, f_{t^{\prime}},\left(\mathcal{V}_{\sigma}^{r}\right)_{0}\right]\right) \\
& =\mathbb{C}\left(\left[f_{p}, f_{t^{\prime}}, v^{s},\left(\mathcal{V}_{\sigma}^{r}\right)_{0}\right]-\left[f_{p}, v^{s},\left(\mathcal{V}_{\sigma}^{r}\right)_{-\alpha_{t^{\prime}}}\right]\right) \\
& \subseteq \sum_{\tau \in \mathbb{Z}^{\nu}}\left(\mathcal{G}_{\tau}\right)_{0}+\sum_{1 \leq k \leq m} \sum_{\tau \in \mathbb{Z}^{\nu}}\left(\mathcal{V}_{\tau}^{k}\right)_{0} .
\end{aligned}
$$

Now set $v_{0,1}:=\left[f_{3}, v_{\sigma, 3}^{r}\right], v_{0,2}:=\left[f_{4}, v_{\sigma, 4}^{r}\right]$. As $\psi^{s}$ and $\psi_{\sigma}^{r}$ are $\mathcal{G}$-module homomorphisms, 5.3 implies that

$$
\left[\left[f_{j}, f_{i}\right], v_{p}^{s}\right]=a_{1}\left[f_{4}, v_{4}^{s}\right], \quad v_{n_{s}}^{s}=a_{3}\left[e_{t^{\prime}}, v_{p}^{s}\right], \quad\left[f_{t^{\prime}}, f_{j}, f_{i}, b^{\prime} v_{0,1}+b v_{0,2}\right]=y v_{-\alpha_{n_{s}}}^{r} .
$$

Next we note that $b^{\prime} v_{0,1}+b v_{0,2} \in\left(\mathcal{V}_{\sigma}^{r}\right)_{0}$, so $\left[f_{j}, f_{i}, b^{\prime} v_{0,1}+b v_{0,2}\right] \in\left(\mathcal{V}_{\sigma}^{r}\right)_{-\alpha_{i}-\alpha_{j}}$ and so $\left[h_{t^{\prime}}, f_{j}, f_{i}, b^{\prime} v_{0,1}+b v_{0,2}\right]=\left[f_{j}, f_{i}, b^{\prime} v_{0,1}+b v_{0,2}\right]$. Also as $\alpha_{j}$ and $\alpha_{i}+\alpha_{j}-\alpha_{t^{\prime}}$ are not short roots, $\left[e_{t^{\prime}}, f_{j}, f_{i}, b^{\prime} v_{0,1}+b v_{0,2}\right]=0$ and $\left[f_{j}, b^{\prime} v_{0,1}+b v_{0,2}\right]=0$. Together 
with (5.4) and (5.5), this implies that

$$
\begin{aligned}
{\left[v^{s}, v_{\sigma,-n_{s}}^{r}\right] } & =\frac{a_{3}}{y}\left[\left[e_{t^{\prime}}, v_{p}^{s}\right], f_{t^{\prime}}, f_{j}, f_{i}, b^{\prime} v_{0,1}+b v_{0,2}\right] \\
& =\frac{a_{3}}{y}\left(\left[e_{t^{\prime}}, v_{p}^{s}, f_{t^{\prime}}, f_{j}, f_{i}, b^{\prime} v_{0,1}+b v_{0,2}\right]-\left[v_{p}^{s}, e_{t^{\prime}}, f_{t^{\prime}}, f_{j}, f_{i}, b^{\prime} v_{0,1}+b v_{0,2}\right]\right) \\
& =\frac{a_{3}}{y}\left(X-\left[v_{p}^{s}, h_{t^{\prime}}, f_{j}, f_{i}, b^{\prime} v_{0,1}+b v_{0,2}\right]-\left[v_{p}^{s}, f_{t^{\prime}}, e_{t^{\prime}}, f_{j}, f_{i}, b^{\prime} v_{0,1}+b v_{0,2}\right]\right) \\
& =\frac{a_{3}}{y}\left(X-\left[v_{p}^{s}, f_{j}, f_{i}, b^{\prime} v_{0,1}+b v_{0,2}\right]\right) \\
& =\frac{a_{3}}{y}\left(X-\left[v_{p}^{s},\left[f_{j}, f_{i}\right], b^{\prime} v_{0,1}+b v_{0,2}\right]-\left[v_{p}^{s}, f_{i}, f_{j}, b^{\prime} v_{0,1}+b v_{0,2}\right]\right) \\
& =\frac{a_{3}}{y}\left(X-\left[\left[v_{p}^{s},\left[f_{j}, f_{i}\right]\right], b^{\prime} v_{0,1}+b v_{0,2}\right]-\left[\left[f_{j}, f_{i}\right], v_{p}^{s}, b^{\prime} v_{0,1}+b v_{0,2}\right]\right) \\
& \in \frac{a_{3}}{y}\left(X+a_{1}\left[\left[f_{4}, v_{4}^{s}\right], b^{\prime} v_{0,1}+b v_{0,2}\right]-\left[\left[f_{j}, f_{i}\right], v_{p}^{s},\left(\mathcal{V}_{\sigma}^{r}\right)_{0}\right]\right) \\
& \equiv \frac{a_{1} a_{3}}{y}\left[\left[f_{4}, v_{2}^{s}\right], b^{\prime} v_{0,1}+b v_{0,2}\right]\left(\bmod \sum_{\tau \in \mathbb{Z}^{\nu}}\left(\mathcal{G}_{\tau}\right)_{0}+\sum_{1 \leq k \leq m} \sum_{\tau \in \mathbb{Z}^{\nu}}\left(\mathcal{V}_{\tau}^{k}\right)_{0}\right) .
\end{aligned}
$$

On the other hand, using (vi), we have $\left[f_{n_{s}}, v^{s}, b^{\prime} v_{0,1}+b v_{0,2}\right] \in \sum_{\tau \in \mathbb{Z}^{\nu}}\left(\mathcal{G}_{\tau}\right)_{0}+$ $\sum_{1 \leq k \leq m} \sum_{\tau \in \mathbb{Z}^{\nu}}\left(\mathcal{V}_{\tau}^{k}\right)_{0}$, so the Jacobi identity implies that

$$
\begin{aligned}
{\left[v^{s}, v_{\sigma,-n_{s}}^{r}\right] } & =\frac{1}{z}\left[v^{s}, f_{n_{s}}, b^{\prime} v_{0,1}+b v_{0,2}\right] \\
& =\frac{1}{z}\left(\left[\left[v^{s}, f_{n_{s}}\right], b^{\prime} v_{0,1}+b v_{0,2}\right]+\left[f_{n_{s}}, v^{s}, b^{\prime} v_{0,1}+b v_{0,2}\right]\right) \\
& =\frac{a_{2}}{z}\left(\left[\left[v_{3}^{s}, f_{3}\right], b^{\prime} v_{0,1}+b v_{0,2}\right]+\left[f_{n_{s}}, v^{s}, b^{\prime} v_{0,1}+b v_{0,2}\right]\right) \\
& \equiv \frac{a_{2}}{z}\left[\left[v_{3}^{s}, f_{3}\right], b^{\prime} v_{0,1}+b v_{0,2}\right]\left(\bmod \sum_{\tau \in \mathbb{Z}^{\nu}}\left(\mathcal{G}_{\tau}\right)_{0}+\sum_{1 \leq k \leq m} \sum_{\tau \in \mathbb{Z}^{\nu}}\left(\mathcal{V}_{\tau}^{k}\right)_{0}\right) .
\end{aligned}
$$

We have the following congruences modulo $\sum_{\tau \in \mathbb{Z}^{\nu}}\left(\mathcal{G}_{\tau}\right)_{0}+\sum_{1 \leq k \leq m} \sum_{\tau \in \mathbb{Z}^{\nu}}\left(\mathcal{V}_{\tau}^{k}\right)_{0}$ :

$$
\begin{aligned}
\left(\frac{-z a^{\prime}}{a_{2}}+\frac{y a}{a_{3} a_{1}}\right)\left[v^{s}, v_{\sigma,-\alpha_{n_{s}}}^{r}\right] & \equiv \frac{-z a^{\prime}}{a_{2}}\left[v^{s}, v_{\sigma,-\alpha_{n_{s}}}^{r}\right]+\frac{y a}{a_{3} a_{1}}\left[v^{s}, v_{\sigma,-\alpha_{n_{s}}}^{r}\right] \\
& \equiv\left[a^{\prime}\left[f_{3}, v_{1}^{s}\right]+a\left[f_{4}, v_{2}^{s}\right], b^{\prime}\left[f_{3}, v_{\sigma, 3}^{r}\right]+b\left[f_{4}, v_{\sigma, 4}^{r}\right]\right] \in \mathcal{D} .
\end{aligned}
$$

For types other than $F_{4}$, we first note that $\mathbb{C}\left[f_{\ell}, v_{\ell}^{s}\right]=\mathbb{C}\left[f_{n_{s}}, v^{s}\right]$. We have

$$
\begin{aligned}
{\left[v^{s}, v_{\sigma,-n_{s}}^{r}\right] \in\left[v^{s},\left[f_{n_{s}},\left(\mathcal{V}_{\sigma}^{r}\right)_{0}\right]\right] } & =\mathbb{C}\left[v^{s},\left[f_{n_{s}},\left[f_{\ell}, v_{\ell, \sigma}^{r}\right]\right]\right] \\
& \subseteq \mathbb{C}\left[\left[v^{s}, f_{n_{s}}\right],\left[f_{\ell}, v_{\ell, \sigma}^{r}\right]\right]+\mathbb{C}\left[f, v^{s},\left[f_{\ell}, v_{\ell, \sigma}^{r}\right]\right] \\
& \subseteq \mathbb{C}\left[\left[f_{\ell}, v_{\ell}^{s}\right],\left[f_{\ell}, v_{\ell, \sigma}^{r}\right]\right]+\mathbb{C}\left[f_{n_{s}}, v^{s},\left[f_{\ell}, v_{\ell, \sigma}^{r}\right]\right]
\end{aligned}
$$

Now we are done using (vi). 
(viii), (ix) Use the same argument as in [You, Lemma 2.5].

(x) We fix $p, q \in J_{m}$ and $\gamma, \delta \in \mathbb{Z}$ and show that

$$
\left[e_{i}, D_{\gamma, \delta}^{p, q}\right]=\left[f_{i}, D_{\gamma, \delta}^{p, q}\right]=0 ; \quad 1 \leq i \leq \ell .
$$

We know that $\mathcal{V}_{\gamma}^{p}, \mathcal{V}_{\delta}^{q}$ are $\mathcal{G}$-modules, so for $n_{\ell}+1 \leq j \leq \ell,\left[f_{j}, v_{\gamma, j}^{p}\right] \in\left(\mathcal{V}_{\gamma}^{p}\right)_{0}$ and $\left[f_{j}, v_{\delta, j}^{q}\right] \in\left(\mathcal{V}_{\delta}^{q}\right)_{0}$, therefore $\left[e_{i}, f_{j}, v_{\gamma, j}^{p}\right]=0$ and $\left[e_{i}, f_{j}, v_{\delta, j}^{q}\right]=0$ for $1 \leq i \leq n_{\ell}$, which together with the Jacobi identity implies that

$$
\left[e_{i}, D_{\gamma, \delta}^{p, q}\right]=\left[f_{i}, D_{\gamma, \delta}^{p, q}\right]=0 ; \quad 1 \leq i \leq n_{\ell} .
$$

Therefore it remains to prove that

$$
\left[e_{i}, D_{\gamma, \delta}^{p, q}\right]=\left[f_{i}, D_{\gamma, \delta}^{p, q}\right]=0 ; \quad n_{\ell}+1 \leq i \leq \ell .
$$

We first consider type $F_{4}$. In this case the only simple short roots appearing in our fixed basis are $\alpha_{3}, \alpha_{4}$. Therefore it is enough to show that $\left[e_{i}, D_{\gamma, \delta}^{p, q}\right]=0$ for $i=3,4$. Using the Jacobi identity, the Claim and (3.21), we have

$$
\begin{aligned}
{\left[e_{3}, D_{\gamma, \delta}^{p, q}\right]=} & {\left[e_{3},\left[a^{\prime}\left[f_{3}, v_{\gamma, 3}^{p}\right]+a\left[f_{4}, v_{\gamma, 4}^{p}\right], b^{\prime}\left[f_{3}, v_{\delta, 3}^{q}\right]+b\left[f_{4}, v_{\delta, 4}^{q}\right]\right]\right] } \\
= & \left(a_{3}^{\prime \prime} a^{\prime}+b_{3}^{\prime \prime} a\right)\left[v_{\gamma, 3}^{p}, b^{\prime}\left[f_{3}, v_{\delta, 3}^{q}\right]+b\left[f_{4}, v_{\delta, 4}^{q}\right]\right] \\
& -\left(a_{3}^{\prime \prime} b^{\prime}+b_{3}^{\prime \prime} b\right)\left[v_{\gamma, 3}^{q}, a^{\prime}\left[f_{3}, v_{\delta, 3}^{p}\right]+a\left[f_{4}, v_{\delta, 4}^{p}\right]\right] \\
= & \left(a_{3}^{\prime \prime} a^{\prime}+b_{3}^{\prime \prime} a\right) b\left[v_{\gamma, 3}^{p},\left[f_{4}, v_{\delta, 4}^{q}\right]\right]-\left(a_{3}^{\prime \prime} b^{\prime}+b_{3}^{\prime \prime} b\right) a\left[v_{\gamma, 3}^{q},\left[f_{4}, v_{\delta, 4}^{p}\right]\right] \\
= & 0 .
\end{aligned}
$$

Also

$$
\begin{aligned}
{\left[e_{4}, D_{\gamma, \delta}^{p, q}\right]=} & {\left[e_{4},\left[a^{\prime}\left[f_{3}, v_{\gamma, 3}^{p}\right]+a\left[f_{4}, v_{\gamma, 4}^{p}\right], b^{\prime}\left[f_{3}, v_{\delta, 3}^{q}\right]+b\left[f_{4}, v_{\delta, 4}^{q}\right]\right]\right] } \\
= & \left(a_{4}^{\prime \prime} a^{\prime}+b_{4}^{\prime \prime} a\right)\left[v_{\gamma, 4}^{p}, b^{\prime}\left[f_{3}, v_{\delta, 3}^{q}\right]+b\left[f_{4}, v_{\delta, 4}^{q}\right]\right] \\
& -\left(a_{4}^{\prime \prime} b^{\prime}+b_{4}^{\prime \prime} b\right)\left[v_{\gamma, 4}^{q}, a^{\prime}\left[f_{3}, v_{\delta, 3}^{p}\right]+a\left[f_{4}, v_{\delta, 4}^{p}\right]\right] \\
= & \left.\left(a_{4}^{\prime \prime} a^{\prime}+b_{4}^{\prime \prime} a\right) b^{\prime}\left[v_{\gamma, 4}^{q},\left[f_{3}, v_{\delta, 3}^{q}\right]\right]-\left(a_{4}^{\prime \prime} b^{\prime}+b_{4}^{\prime \prime} b\right) a^{\prime}\left[v_{\gamma, 4}^{q},\left[f_{3}, v_{\delta, 3}^{p}\right]\right]\right] \\
= & 0 .
\end{aligned}
$$

Using (R9) together with 2.13 , we get

$$
\left[v^{p}, v_{ \pm j}^{q}\right]=\left[v^{q}, v_{ \pm j}^{p}\right] ; \quad n_{\ell+1} \leq j \leq n_{s} .
$$

We note that in the cases under consideration, $\left(\mathcal{V}_{\gamma}^{q}\right)_{0}$ is a one-dimensional subspace of $\mathcal{V}_{\gamma}^{q}$. Let $n_{\ell}+1 \leq i \leq \ell$. Then there is $x \in \mathbb{C}$ such that

$$
x\left[f_{i}, v_{\gamma, i}^{q}\right]=\left[f_{\ell}, v_{\gamma, \ell}^{q}\right] \quad \text { and } \quad x\left[f_{i}, v_{\delta, i}^{p}\right]=\left[f_{\ell}, v_{\delta, \ell}^{p}\right] .
$$


Now using Propositions 3.3 and 3.1(iii), and [5.6), we have $\left[f_{n_{s}}, v_{\delta}^{p}, v_{\gamma, i}^{q}\right]=$ $\left[f_{n_{s}}, v_{\gamma}^{q}, v_{\delta, i}^{p}\right]$. This together with the Jacobi identity implies that

$$
\begin{aligned}
{\left[e_{i},\left[f_{\ell}, v_{\delta, \ell}^{p}\right],\left[f_{\ell}, v_{\gamma, \ell}^{q}\right]\right]=} & {\left[\left[f_{n_{s}}, v_{\delta}^{p}\right],\left[e_{i},\left[f_{n_{s}}, v_{\gamma}^{q}\right]\right]\right]-\left[\left[f_{n_{s}}, v_{\gamma}^{q}\right],\left[e_{i},\left[f_{n_{s}}, v_{\delta}^{p}\right]\right]\right] } \\
= & x\left[\left[f_{n_{s}}, v_{\delta}^{p}\right],\left[e_{i},\left[f_{i}, v_{\gamma, i}^{q}\right]\right]-x\left[\left[f_{n_{s}}, v_{\gamma}^{q}\right],\left[e_{i},\left[f_{i}, v_{\delta, i}^{p}\right]\right]\right.\right. \\
= & x\left[\left[f_{n_{s}}, v_{\delta}^{p}\right],\left[\left[e_{i}, f_{i}\right], v_{\gamma, i}^{q}\right]\right]-x\left[\left[f_{n_{s}}, v_{\gamma}^{q}\right],\left[\left[e_{i}, f_{i}\right], v_{\delta, i}^{p}\right]\right] \\
= & 2 x\left[\left[f_{n_{s}}, v_{\delta}^{p}\right], v_{\gamma, i}^{q}\right]-2 x\left[\left[f_{n_{s}}, v_{\gamma}^{q}\right], v_{\delta, i}^{p}\right] \\
= & 2 x\left(\left[f_{n_{s}}, v_{\delta}^{p}, v_{\gamma, i}^{q}\right]-\left[v_{\delta}^{p}, f_{n_{s}}, v_{\gamma, i}^{q}\right]\right. \\
& \left.-\left[f_{n_{s}}, v_{\gamma}^{q}, v_{\delta, i}^{p}\right]+\left[v_{\gamma}^{q}, f_{n_{s}}, v_{\delta, i}^{p}\right]\right) \\
= & 2 x\left(-\left[v_{\delta}^{p}, f_{n_{s}}, v_{\gamma, i}^{q}\right]+\left[v_{\gamma}^{q}, f_{n_{s}}, v_{\delta, i}^{p}\right]\right) .
\end{aligned}
$$

Now if $\alpha_{\ell}-\alpha_{i}$ is not a short root then $\left[f_{n_{s}}, v_{\delta, i}^{p}\right]=\left[f_{n_{s}}, v_{\gamma, i}^{q}\right]=0$ and so we are done, otherwise there are $n_{\ell}+1 \leq k \leq n_{s}$ and $y \in \mathbb{C}$ such that

$$
\left[f_{n_{s}}, v_{\delta, i}^{p}\right]=y v_{\delta, \pm k}^{p} \quad \text { and } \quad\left[f_{n_{s}}, v_{\gamma, i}^{q}\right]=y v_{\gamma, \pm k}^{q} .
$$

This together with (5.7), Propositions 3.3 and 3.1(iii), and (5.6) implies that

$$
\left[e_{i}, D_{\gamma, \delta}^{p, q}\right] \in \mathbb{C}\left[e_{i},\left[f_{\ell}, v_{\delta, \ell}^{p}\right],\left[f_{\ell}, v_{\gamma, \ell}^{q}\right]\right]=\mathbb{C}\left(-\left[v_{\delta}^{p}, v_{\gamma, \pm k}^{q}\right]+\left[v_{\gamma}^{q}, v_{\delta, \pm k}^{p}\right]\right)=0 .
$$

Using the same argument as above, one can show that

$$
\left[f_{i}, D_{\gamma, \delta}^{p, q}\right]=0 .
$$

(xi) Let $i \in J_{\ell}$ and $\gamma, \delta \in \mathbb{Z}^{\nu}$ and fix $j \in J_{\ell}, r \in J_{m}$ and $a \in J_{\nu}$. We need to prove

$$
\left[e_{j},\left[h_{i, \gamma}, h_{i, \delta}\right]\right]=\left[f_{j},\left[h_{i, \gamma}, h_{i, \delta}\right]\right]=\left[h_{j, a}^{ \pm},\left[h_{i, \gamma}, h_{i, \delta}\right]\right]=\left[v^{p},\left[h_{i, \gamma}, h_{i, \delta}\right]\right]=0 .
$$

Using the Jacobi identity together with Proposition 3.4(ii), we have

$$
\begin{aligned}
{\left[e_{j},\left[h_{i, \gamma}, h_{i, \delta}\right]\right] } & =\left[h_{i, \delta}, h_{i, \gamma}, e_{j}\right]-\left[h_{i, \gamma}, h_{i, \delta}, e_{j}\right]=\alpha_{j}\left(h_{i}\right)\left(\left[h_{i, \delta}, e_{j, \gamma}\right]-\left[h_{i, \gamma}, e_{j, \delta}\right]\right) \\
& =\left(\alpha_{j}\left(h_{i}\right)\right)^{2}\left(e_{j, \gamma+\delta}-e_{j, \gamma+\delta}\right)=0 .
\end{aligned}
$$

The proof for the second term is similar and for the last one it is immediate using Proposition 3.4(vi). Now it remains to prove $\left[h_{j, a}^{ \pm},\left[h_{i, \gamma}, h_{i, \delta}\right]\right]=0$. For this we first prove $\left[h_{i, a}^{ \pm}, h_{i, b}^{ \pm}, h_{i, \delta}\right]=0$ for all $b \in J_{\nu}$. Fix $b \in J_{\nu}$ and use Proposition 3.4(v),(iii) to get

$$
\begin{aligned}
{\left[f_{i, a}^{ \pm}, h_{i, b}^{ \pm}, h_{i, \delta}\right] } & =2\left[f_{i, a}^{ \pm},\left[e_{i, b}^{ \pm}, f_{i, \delta}\right]-h_{i, \delta+\gamma_{b}^{ \pm}}\right]=2\left[f_{i, a}^{ \pm},\left[e_{i, b}^{ \pm}, f_{i, \delta}\right]\right]-4 f_{i, \delta+\gamma_{a}^{ \pm}+\gamma_{b}^{ \pm}} \\
& =2\left[f_{i, \delta}, e_{i, b}^{ \pm}, f_{i, a}^{ \pm}\right]-2\left[e_{i, b}^{ \pm}, f_{i, \delta}, f_{i, a}^{ \pm}\right]-4 f_{i, \delta+\gamma_{b}^{ \pm}+\gamma_{a}^{ \pm}} \\
& =2\left[f_{i, \delta}, e_{i, b}^{ \pm}, f_{i, a}^{ \pm}\right]-4 f_{i, \delta+\gamma_{b}^{ \pm}+\gamma_{a}^{ \pm}}
\end{aligned}
$$


But again using Proposition 3.4(v),(iii), we obtain

$$
\begin{aligned}
2\left[f_{i \delta},\left[e_{i, b}^{ \pm}, f_{i, a}^{ \pm}\right]\right] & =2\left[f_{i \delta},\left[e_{i, b}^{ \pm}, f_{i, \gamma_{a}^{ \pm}}\right]\right]=\left[f_{i, \delta},\left[h_{i, b}^{ \pm}, h_{i, a}^{ \pm}\right]\right]+2\left[f_{i, \delta}, h_{i, \gamma_{a}^{ \pm}+\gamma_{b}^{ \pm}}\right] \\
& =\left[h_{i, a}^{ \pm}, h_{i, b}^{ \pm}, f_{i, \delta}\right]-\left[h_{i, b}^{ \pm}, h_{i, a}^{ \pm}, f_{i, \delta}\right]+4 f_{i, \gamma_{b}^{ \pm}+\gamma_{b}^{ \pm}+\delta} \\
& =-2\left[h_{i, a}^{ \pm}, f_{i, \delta+\gamma_{b}^{ \pm}}\right]+2\left[h_{i, b}^{ \pm}, f_{i, \delta+\gamma_{a}^{ \pm}}\right]+4 f_{i, \gamma_{b}^{ \pm}+\gamma_{j}^{ \pm}+\delta} \\
& =4 f_{i, \delta+\gamma_{a}^{ \pm}+\gamma_{b}^{ \pm}}-4 f_{i, \delta+\gamma_{a}^{ \pm}+\gamma_{b}^{ \pm}}+4 f_{i, \gamma_{b}^{ \pm}+\gamma_{a}^{ \pm}+\delta}=4 f_{i, \gamma_{b}^{ \pm}+\gamma_{a}^{ \pm}+\delta} .
\end{aligned}
$$

Therefore

$$
\left[f_{i, a}^{ \pm}, h_{i, b}^{ \pm}, h_{i, \delta}\right]=0
$$

and so by (3.16), the Jacobi identity and the first part of the proof, we have

$$
\begin{aligned}
{\left[h_{i, a}^{ \pm}, h_{i, b}^{ \pm}, h_{i, \delta}\right] } & =\left[\left[e_{i}, f_{i, a}^{ \pm}\right], h_{i, b}^{ \pm}, h_{i, \delta}\right] \\
& =\left[e_{i}, f_{i, a}^{ \pm}, h_{i, b}^{ \pm}, h_{i, \delta}\right]-\left[f_{i, a}^{ \pm}, e_{i}, h_{i, b}^{ \pm}, h_{i, \delta}\right]=0-0=0 .
\end{aligned}
$$

Now using Proposition 3.4(iv), the information obtained and the fact that (3.1) is a generating set for the Lie algebra, one can conclude that $\left[h_{j, b}^{ \pm}, h_{i, \delta}\right] \in Z(\tilde{\mathcal{L}})$ for all $b \in J_{\nu}$. Similarly one can get $\left[h_{j, b}^{ \pm}, h_{i, \gamma}\right] \in Z(\tilde{\mathcal{L}})$. Therefore the Jacobi identity implies that

$$
\left[h_{j, a}^{ \pm},\left[h_{i, \gamma}, h_{i, \delta}\right]\right]=\left[h_{i, \gamma},\left[h_{j, a}^{ \pm}, h_{i, \delta}\right]\right]-\left[h_{i, \delta},\left[h_{j, a}^{ \pm}, h_{i, \gamma}\right]\right]=0 .
$$

This completes the proof.

\section{Acknowledgements}

The first and the third authors would like to thank the Center of Excellence for Mathematics, University of Isfahan, for partial support of this work. Research of the first author was also supported in part by a grant from IPM (No. 89170216).

\section{References}

[AABGP] B. Allison, S. Azam, S. Berman, Y. Gao and A. Pianzola, Extended affine Lie algebras and their root systems, Mem. Amer. Math. Soc. 603 (1997), 122 pp. Zbl 0879.17012 MR 1376741

[ABG] B. Allison, G. Benkart and Y. Gao, Central extensions of Lie algebras graded by finite root systems, Math. Ann. 316 (2000), 499-527. Zbl 0989.17004 MR 1752782

[ABGP] B. Allison, S. Berman, Y. Gao and A. Pianzola, A characterization of affine KacMoody Lie algebras, Comm. Math. Phys. 185 (1997), 671-688. Zbl 0879.17013 MR 1463057

[AG] B. Allison and Y. Gao, The root system and the core of an extended affine Lie algebra, Selecta Math. (N.S.) 7 (2001), 149-212. Zbl 1058.17012 MR 1860013

[AK] S. Azam and V. Khalili, Lie tori and their fixed point subalgebras, Algebra Colloq. 16 (2009), 381-396. Zbl 1184.17009 MR 2536764 
[AKY] S. Azam, V. Khalili and M. Yousofzadeh, Extended affine root systems of type BC, J. Lie Theory 15 (2005), 145-181. Zbl 1100.17003 MR 2115234

[BZ] G. Benkart and E. Zelmanov, Lie algebras graded by finite root systems and intersection matrix algebras, Invent. Math. 126 (1996), 1-45. Zbl 0871.17024 MR 1408554

[BGK] S. Berman, Y. Gao and Y. Krylyuk, Quantum tori and the structure of elliptic quasi-simple Lie algebras, J. Funct. Anal. 135 (1996), 339-386. Zbl 0847.17009 MR 1370607

[BGKN] S. Berman, Y. Gao, Y. Krylyuk and E. Neher, The alternative torus and the structure of elliptic quasi-simple Lie algebras of type $A_{2}$, Trans. Amer. Math. Soc. 347 (1995), 4315-4363. Zbl 0847.17010 MR 1303115

$[\mathrm{BM}] \quad$ S. Berman and R. V. Moody, Lie algebras graded by finite root systems and the intersection matrix algebras of Slodowy, Invent. Math. 108 (1992), 323-347. Zbl 0778.17018 MR 1161095

[H] J. E. Humphreys, Introduction to Lie algebras and representation theory, Springer, New York, 1972. Zbl 0254.17004 MR 0323842

[J] N. Jacobson, Structure and representations of Jordan algebras, Amer. Math. Soc. Colloq. Publ. 39, Amer. Math. Soc., Providence, RI, 1968. Zbl 0218.17010 MR 0251099

[Ka] C. Kassel, Kähler differentials and coverings of complex simple Lie algebras extended over a commutative algebra, J. Pure Appl. Algebra 34 (1984), 265-275. Zbl 0549.17009 MR 0772062

[MP] R. V. Moody and A. Pianzola, Lie algebras with triangular decomposition, WileyInterscience, New York, 1995. Zbl 0874.17026 MR 1323858

[MRY] R. Moody, S. E. Rao and T. Yokonuma, Toroidal Lie algebras and vertex representations, Geom. Dedicata 35 (1990), 283-307. Zbl 0704.17011 MR 1066569

[SY] K. Saito and D. Yoshii, Extended affine root systems IV (Simply-laced elliptic Lie algebras), Publ. Res. Inst. Math. Sci. 36 (2000), 385-421. Zbl 0987.17012 MR 1781435

[Sc] R. D. Schafer, An introduction to nonassociative algebras, Academic Press, New York, 1966. Zbl 0145.25601 MR 0210757

[Yam] H. Yamane, A Serre-type theorem for the elliptic Lie algebras with rank $\geq 2$, Publ. Res. Inst. Math. Sci. 40 (2004), 441-469. Zbl 1142.17308

[Yos] Y. Yoshii, Lie tori-a simple characterization of extended affine Lie algebras, Publ. Res. Inst. Math. Sci. 42 (2006), 739-762. Zbl 1148.17017 MR 2266995

[You] M. Yousofzadeh, A presentation of Lie tori of type $B_{\ell}$, Publ. Res. Inst. Math. Sci. 44 (2008), 1-44. Zbl pre05289830 MR 2405865 